\title{
$F$-singularities in families
}

\author{
Zsolt Patakfalvi, Karl Schwede and Wenliang Zhang
}

\begin{abstract}
We study the behavior of test ideals and $F$-singularities in families. In particular, we obtain generic (and non-generic) restriction theorems for test ideals and non- $F$-pure ideals which imply, for example, openness of most of the $F$-singularity classes when the relative canonical sheaf is $\mathbb{Q}$-Cartier. Additionally, we study the global behavior of certain canonical linear systems (induced by the Frobenius morphism) associated to adjoint line bundles, in families. As a consequence, we obtain some positivity results for pushforwards of some adjoint line bundles and for certain subsheaves of these.
\end{abstract}

\section{Introduction}

In [Kun69], Kunz proved that a scheme over a field of characteristic $p>0$ is regular if and only if the Frobenius (endo)morphism is flat, which initiated the study of singularities using the Frobenius morphism. Classes of singularities defined via the Frobenius morphism are usually referred to as $F$-singularities. Since Kunz's work, the study of $F$-singularities has become an active research area; see [HR76, GW77, Fed83, MR85, RR85, Sri91, Smi97, Har98, MS97, HY03, Tak04]. However, the methods of $F$-singularities have been widely applied to global geometry over a field of positive characteristic only recently [Sch14, Mus13, Hac15, MS14, Zha14, CHMS14, Pat14, HX15, Tan15], at least outside of special classes of varieties [MR85, BK05]. A large field within global geometry is moduli theory, which requires understanding how varieties behave in flat families. In this direction, there have not been any positive results on the behavior of test ideals in families. We fill this gap.

Previously, F-rational, Cohen-Macaulay, F-injective, and (Gorenstein) F-pure singularities have been studied in such a context [Has01, SZ09, Has10]. Additionally, [MY09, Example 4.7] showed that the test ideal $\tau$ does not satisfy the generic restriction theorem, at least as stated for multiplier ideals [Laz04b, Theorem 9.5.35]. We develop tools tackling this issue, obtaining generic (and non-generic) restriction theorems for test ideals; see Theorem A below.

In a flat family $f: X \rightarrow V$, the absolute Frobenius morphism on $X$ does not restrict to the Frobenius morphism on each fiber. Thus, we systematically study the relative Frobenius

Received 3 October 2013, accepted in final form 16 March 2017.

2010 Mathematics Subject Classification 14B05, 13A35, 14D05, 14F18, 13B40.

Keywords: non- $F$-pure ideal, test ideal, $F$-pure, strongly $F$-regular, $F$-singularities, flat families.

This journal is (C) Foundation Compositio Mathematica 2018. This article is distributed with Open Access under the terms of the Creative Commons Attribution Non-Commercial License, which permits non-commercial reuse, distribution, and reproduction in any medium, provided that the original work is properly cited. For commercial re-use, please contact the Foundation Compositio Mathematica.

The second author was partially supported by a fellowship from the Sloan Foundation as well as NSF grant DMS \#1064485, NSF FRG Grant DMS \#1265261/1501115 and NSF CAREER Grant DMS \#1252860/1501102.

The third author was partially supported by the NSF grants DMS \#1068946/\#1247354 and \#1606414.

The material is based upon work supported by the National Science Foundation under Grant \#0932078 000 , while the second and third authors were in residence at the Mathematical Science Research Institute in Berkeley, California, during the spring semester 2013. 


\section{$F$-SINGULARITIES IN FAMILIES}

morphism $^{1} X^{\prime} \rightarrow X \times_{V} V^{\prime}$, where $X^{\prime} \rightarrow X$ and $V^{\prime} \rightarrow V$ are the Frobenius morphisms of $X$ and $V$, respectively. Its fibers over $V$ are morphisms between thickenings of the fibers of $f$. However, its fibers over $V^{\prime}$ are exactly the Frobenius morphisms of fibers of $f$ (at least over points with perfect residue field).

We begin by stating our main result in the local setting. We denote by $X^{n}$ and $V^{n}$ the domains of $n$-iterated Frobenius morphisms of $X$ and $V$, respectively. Let $\tau$ and $\sigma$ denote the test ideal $^{2}$ [HH94] and non-sharply- $F$-pure ideal [FST11, BB11], respectively. These ideals play key roles in the theory of $F$-singularities, the test ideal $\tau$ being the unique smallest non-zero ideal fixed by $p^{-e}$-linear maps and $\sigma$ being the unique largest. We define relative versions of these two ideals by using the relative instead of absolute Frobenius morphism. The iterated relative Frobenius morphism targets different spaces $X \times_{V} V^{n}$, and so we actually define a sequence of ideals, one on each of these spaces. We explain the setup.

Suppose that $f: X \rightarrow V$ is a finite-type, flat, equidimensional, reduced, and S2 and G1 morphism of Noetherian $F$-finite schemes with $V$ integral. Additionally, suppose that $\Delta$ is a $\mathbb{Q}$-divisor on $X$ satisfying suitable conditions such that it can be restricted to fibers and such that $K_{X / V}+\Delta$ is $\mathbb{Q}$-Cartier with index not divisible by $p>0$, so that $\left(p^{e}-1\right)\left(K_{X / V}+\Delta\right)$ is Cartier (see Remark 2.11 for a precise statement). Then, for each integer $n>0$ divisible by $e$, we define ideals $\sigma_{n}(X / V, \Delta)$ and $\tau_{n}(X / V, \Delta) \subseteq \mathscr{O}_{X \times_{V} V^{n}}$ called the relative non-F-pure and relative test ideals, respectively.

Our main local theorem is that these ideals restrict to absolute non- $F$-pure and absolute test ideals on all of the geometric fibers and can be used to prove generic restriction theorems for the usual absolute test ideals on $X \times_{V} V^{n}$, at least if $V$ is regular.

Theorem A (Theorem 3.10, Corollaries 3.22, 4.8, and 4.14). With notation as above, there exists an $N>0$ such that for every perfect point ${ }^{3} s \in V$ and every $n \geqslant N$,

$$
\sigma_{n e}(X / V, \Delta) \cdot \mathscr{O}_{X_{s} n e}=\sigma\left(X_{s},\left.\Delta\right|_{X_{s}}\right)
$$

and

$$
\tau_{n e}(X / V, \Delta) \cdot \mathscr{O}_{X_{s} n e}=\tau\left(X_{s},\left.\Delta\right|_{X_{s}}\right),
$$

where $X_{s^{n e}}$ is the fiber of $X \times_{V} V^{n e} \rightarrow V^{n e}$ over $s^{n e} \in V^{n e}$, which is isomorphic to $X_{s}$ since $k(s)$ is perfect. Additionally, both $\sigma_{n}$ and $\tau_{n e}$ map surjectively onto their arbitrary base changes.

Furthermore, if $V$ is regular and $N$ is sufficiently large, then for all perfect points $s \in V$, the absolute non-F-pure ideal restricts to all of the fibers for $n \geqslant N$ :

$$
\sigma\left(X \times_{V} V^{n e}, \Delta \times_{V} V^{n e}\right) \cdot \mathscr{O}_{X_{s} n e}=\sigma\left(X_{s},\left.\Delta\right|_{X_{s}}\right),
$$

and at least for an open dense set of the base $U \subseteq V$, the same holds for the absolute test ideal:

$$
\tau\left(X \times_{V} V^{n e}, \Delta \times_{V} V^{n e}\right) \cdot \mathscr{O}_{X_{s} n e}=\tau\left(X_{s},\left.\Delta\right|_{X_{s}}\right)
$$

for all perfect points $s \in U$.

Additionally, we show that over a dense open subset $U$ of $V$ with $W=f^{-1}(U)$, the relative test ideal $\left.\tau_{n e}(X / V, \Delta)\right|_{W}$ coincides with the absolute test ideal $\left.\tau\left(X \times_{V} V^{n e}, \Delta \times_{V} V^{n e}\right)\right|_{W}$ and likewise $\left.\sigma_{n e}(X / V, \Delta)\right|_{W}=\left.\sigma\left(X \times_{V} V^{n e}, \Delta \times_{V} V^{n e}\right)\right|_{W}$, in Theorems 4.12 and 3.13, respectively.

\footnotetext{
${ }^{1}$ This is also called the Radu-André morphism, especially in commutative algebra.

${ }^{2}$ Technically, we are working with what has recently become known as the big test ideal [Hoc07].

${ }^{3}$ By definition, a perfect point $s \in V$ is a morphism $\operatorname{Spec} K \rightarrow V$ from a perfect field $K$; see Definition 2.2.
} 


\section{Z. Patakfalvi, K. Schwede And W. Zhang}

The key method that allows us to prove this result (at least stated for $\sigma_{n}$ ) is that if $m>n$, then $\operatorname{im}\left(\sigma_{n e}(X / V, \Delta) \otimes_{\mathscr{O}_{X \times V} \text { ne }} \mathscr{O}_{X \times V^{m e}} \rightarrow \mathscr{O}_{X \times V^{m e}}\right) \supseteq \sigma_{m e}(X / V, \Delta)$ and there is a dense open subset of the base $V$ over which equality holds for all sufficiently large $n$; this is Proposition 3.3. This stabilization result should be viewed as a relative version of [HS77, Proposition 1.11], [Lyu97, Proposition 4.4], and [Gab04, Remark 13.6].

Remark 1.1. If one replaces the divisor $\Delta$ with the language of principal Cartier algebras, then the previous result still holds without the technical assumptions about divisors from Remark 2.11.

Remark 1.2. There are a number of subtle issues in the statement above that we are suppressing. In particular, $\tau_{n}(X / V, \Delta)$ depends on the choice of some ideal $I$ contained within the test ideal of every fiber and is shown to exist in Proposition 4.7.

A particularly important related question is that of deformation of sharply $F$-pure singularities in flat families with $\mathbb{Q}$-Cartier relative canonical divisors. This would be important for a positive-characteristic construction of the moduli of stable varieties, also known as the KSBA compactification. In characteristic zero, this is the moduli space given by the log-minimal model program. It classifies log-canonical models, hence birational equivalence classes of varieties of general type, and furthermore it contains some nodal varieties for the compactification. There is a conjectural framework for constructing this moduli space [Kol90]. One of the main ingredients in this framework is to prove that log-canonical singularity deforms in flat families with $\mathbb{Q}$-Cartier relative log-canonical divisor. An important step in this direction in positive characteristic is the corresponding statement for sharply $F$-pure singularities. It is also an important ingredient in an upcoming paper of the first author where he is planning to address the question of the existence of an algebraic space structure on the space of sharply $F$-pure stable varieties. In this paper, we handle the deformation of $F$-pure and $F$-regular singularities. Indeed, openness of $F$-pure and $F$-regular singularities is a direct consequence of Theorem A above.

TheOREm B (Deformation of $F$-pure and F-regular singularities: Corollaries 3.29 and 4.19). Let $f: X \rightarrow V$ and $\Delta$ be as in Theorem A, and, additionally, assume that $f$ is proper. If $s \in V$ is a perfect point and $\left(X_{s},\left.\Delta\right|_{X_{s}}\right)$ is sharply $F$-pure (respectively, strongly $F$-regular ${ }^{4}$ ), then there exists an open set $U \subseteq V$ such that for all $u \in U$, the pair $\left(X_{u},\left.\Delta\right|_{X_{u}}\right)$ is also sharply $F$-pure (respectively, strongly $F$-regular).

We also build relative test submodules and non- $F$-injective submodules of $\omega_{X \times_{V} V^{n e} / V^{n e}}$ and prove restriction theorems like Theorem A for them (Corollaries 5.4 and 5.8). As a consequence, in Theorem 5.13 we re-prove a result of M. Hashimoto [Has01], namely deformation for CohenMacaulay $F$-injectivity and $F$-rationality.

Furthermore, we apply our setup to global questions. One of the reasons for the recent global applications of the $F$-singularity theorem is the lifting theorem shown by the second author in [Sch14, Proposition 5.3]. This theorem can be used to replace some of the lifting arguments that use Kodaira vanishing in characteristic zero. One of the fundamental ideas in [Sch14] is to try to lift only a big enough set of sections of adjoint bundles instead of all the sections. This canonical set of sections for a pair $(X, \Delta)$ with $\left(1-p^{e}\right)\left(K_{X}+\Delta\right)$ Cartier and for a line bundle $M$ is defined as [Sch14, Definition 4.1]

$$
S^{0}(X, \sigma(X, \Delta) \otimes M):=\bigcap_{m>0} \operatorname{im}\left(H^{0}\left(X, F_{*}^{m e} \mathscr{O}_{X}\left(\left(1-p^{m e}\right)\left(K_{X}+\Delta\right)\right) \otimes M\right) \rightarrow H^{0}(X, M)\right) .
$$

\footnotetext{
${ }^{4}$ In which case, you can even remove the "index not divisible by $p$ " assumption from $K_{X / V}+\Delta$; see Corollary 4.20.
} 


\section{$F$-SINGULARITIES IN FAMILIES}

First, we investigate questions about how this canonical space of sections behaves in families, namely semicontinuity, stabilization of the intersection, etc.

Theorem C. Let $f: X \rightarrow V$ and $\Delta$ be as in Theorem $A$, with $f$ projective and $V$ regular. Further, suppose that $M$ is a line bundle on $X$ such that $M-K_{X / V}-\Delta$ is f-ample (where by $M$ we actually mean the Cartier divisor corresponding to $M$ ). Then the following statements hold:

(a) [Corollary 6.19, Examples 6.22, 6.23, and 6.25], cf. [Har98, Example 5.5], [Tan15, Theorem 8.3]. The function

$$
s \mapsto \operatorname{dim}_{k(s)} S^{0}\left(X_{s}, \sigma\left(X_{s}, \Delta_{s}\right) \otimes M_{s}\right)
$$

is not semicontinuous (here $s \in V$ is a perfect point) in either direction; however, there is a dense open subset $U \in V$ such that the function (1.1) is constant on $U$.

(b) [Theorem 6.18]. There exists an $n>0$ such that for all integers $m \geqslant n$ and perfect points $s \in V$,

$$
\begin{aligned}
\operatorname{im}( & \left.H^{0}\left(X_{s}, F_{*}^{m e} \mathscr{O}_{X_{s}}\left(\left(1-p^{m e}\right)\left(K_{X_{s}}+\Delta_{s}\right)\right) \otimes M_{s}\right) \rightarrow H^{0}\left(X_{s}, M_{s}\right)\right) \\
& =S^{0}\left(X_{s}, \sigma\left(X_{s}, \Delta_{s}\right) \otimes M_{s}\right) .
\end{aligned}
$$

(c) [Proposition 6.20]. If there is a perfect point $s_{0} \in V$ such that

$$
H^{0}\left(X_{s_{0}}, M_{s_{0}}\right)=S^{0}\left(X_{s_{0}}, \sigma\left(X_{s_{0}}, \Delta_{s_{0}}\right) \otimes M_{s_{0}}\right),
$$

then there is an open neighborhood $U$ of $s_{0}$ such that $\left.f_{*} M\right|_{U}$ is locally free and compatible with base change and

$$
H^{0}\left(X_{s}, M_{s}\right)=S^{0}\left(X_{s}, \sigma\left(X_{s}, \Delta_{s}\right) \otimes M_{s}\right)
$$

for every perfect point $s \in U$. In particular, the function (1.1) is constant for $s \in U$.

We would like to also mention the following natural question left open by Theorem C.

Question. Can one remove the $f$-ampleness assumption from the statements of Theorem $C$ ?

By Theorem $\mathrm{C}$, it makes sense to talk about the general value of $\operatorname{dim}_{k(s)} S^{0}\left(X_{s}, \sigma\left(X_{s}, \Delta_{s}\right) \otimes\right.$ $M_{s}$ ) in the following theorem.

Theorem D. With assumptions as in Theorem $C$, so that $V$ is projective over a perfect field and $\left(p^{e}-1\right)\left(K_{X}+\Delta\right)$ is Cartier, for every $n \gg 0$, there is a subsheaf $S_{\Delta, n e}^{0} f_{*}(M)$ of $\left(F_{V}^{n e}\right)^{*}\left(f_{*} M\right)$ for which the following statements hold:

(a) [Corollary 6.19]. The rank of $S_{\Delta, n e}^{0} f_{*}(M)$ is the general value of $\operatorname{dim}_{k(s)} S^{0}\left(X_{s}, \sigma\left(X_{s}, \Delta_{s}\right)\right.$ $\left.\otimes M_{s}\right)$.

(b) [Proposition 6.26]. If $M-K_{X / V}-\Delta$ is ample, then $S_{\Delta, n e}^{0} f_{*}(M)$ is globally generated for every $n \gg 0$.

(c) [Theorem 6.31]. If $M-K_{X / V}-\Delta$ is nef, then $S_{\Delta, n e}^{0} f_{*}(M)$ is weakly positive for $n \gg 0$.

(d) [Corollary 6.14]. If $M=Q^{l} \otimes P$, where $Q$ is $f$-ample, then for all $l \gg 0$ and every nef line bundle $P$, we have that $S_{\Delta, n e}^{0} f_{*}(M)$ is contained in $\left(f_{V^{n e}}\right)_{*}\left(\sigma_{n e}(X / V, \Delta) \otimes M_{V^{n e}}\right)$ as subsheaves of $\left(f_{V^{n e}}\right)_{*} M_{V^{n e}}$, and, furthermore, these two subsheaves are generically equal.

In fact, points (a) and (d) are true without the projectivity assumption on $V$. 


\section{Z. Patakfalvi, K. Schwede And W. Zhang}

Further, note that if $V$ in Theorem $\mathrm{D}$ is a curve, if $M-K_{X / V}-\Delta$ is $f$-ample and nef, and if there is an $s \in V$ such that $H^{0}\left(X_{s}, M_{s}\right)=S^{0}\left(X_{s}, \sigma\left(X_{s}, \Delta_{s}\right)\right)$, then points (a) and (c) of Theorem $\mathrm{D}$ together with point (c) of Theorem $\mathrm{C}$ imply that $f_{*} \mathscr{O}_{X}(M)$ is a nef vector bundle. This strengthens [Pat14, Proposition 3.6]; it removes the cohomology vanishing assumption made there. Of course, our statements are considerably stronger. For example, with the same assumptions except replacing $H^{0}\left(X_{s}, M_{s}\right)=S^{0}\left(X_{s}, \sigma\left(X_{s}, \Delta_{s}\right)\right)$ with $\operatorname{rk} f_{*}(\sigma(X, \Delta) \otimes M)$, which equals the general value of $S^{0}\left(X_{s}, \sigma\left(X_{s}, \Delta_{s}\right)\right)$, we obtain that $f_{*}(\sigma(X, \Delta) \otimes M)$ is nef. In fact, the following even stronger statement can be made.

Theorem E (Corollary 6.39). With assumptions as in Theorem $\mathrm{C}$, so that $V$ is projective over a perfect field, assume that $M-K_{X / V}-\Delta$ is nef and $f$-ample and that $\operatorname{rk} S^{0} f_{*}(\sigma(X, \Delta) \otimes M)$ equals the general value of $H^{0}\left(X_{s}, \sigma\left(X_{s}, \Delta_{s}\right) \otimes M_{s}\right)$. Then $S^{0} f_{*}(\sigma(X, \Delta) \otimes M)$ is weakly positive. In particular, if $V$ is a smooth curve, then it is a nef vector bundle.

Further, we show how $S_{\Delta, n e}^{0} f_{*}(M)$ relates to the other similar notion $S^{0} f_{*}(\sigma(X, \Delta) \otimes M)$ introduced in [HX15, Definition 2.14]. In particular, we obtain that $S^{0} f_{*}(\sigma(X, \Delta) \otimes M)$ does not, in general, restrict to $S^{0}\left(X_{s}, \sigma\left(X_{s}, \Delta_{s}\right) \otimes M_{s}\right)$ for general $s \in S$. Intuitively, though $S^{0} f_{*}(\sigma(X, \Delta)$ $\otimes M)$ is a pushforward, it captures the global geometry of $(X, \Delta)$ rather than the geometry of the fibers. In positive characteristic, these two can differ considerably, essentially because the function field of $V$ is not perfect. Of course, the relative and absolute $S^{0} f_{*}$ are related. We study these similarities and differences in Section 6.6.

Organization. In Section 2, we set up the notation that we will follow throughout the paper, explain the interplay between $p^{-e}$-linear maps and $\mathbb{Q}$-Cartier divisors, and discuss the behavior of such maps and divisors under base changes. In Sections 3, 4, and 5, we introduce the relative non$F$-pure ideals, the relative test ideals, and the relative test submodules, respectively. Applications to $F$-singularities in families are discussed in these three sections. Section 6 is devoted to the behavior of $S^{0}$ (introduced in [Sch14, Definition 4.1]) under base changes. Some semi-positivity results are also proved in this section. Finally, in the appendix, we collect some statements for which we could not find proper references in the generality needed in our paper.

\section{Notation and setup}

Throughout this paper, all schemes are Noetherian and all maps of schemes are separated. We fix the following notation, which is in effect for the entire paper. In particular, for simplicity we do not state it in every statement even though it is assumed.

Notation 2.1. Suppose that $f: X \rightarrow V$ is a flat, equidimensional, and geometrically reduced map $^{5}$ of finite type from a scheme $X$ to an excellent integral scheme $V$ of equal characteristic $p>0$ with a dualizing complex. We write $F^{e}=F_{V}^{e}: V=V^{e} \rightarrow V$ for the absolute $e$-iterated Frobenius morphism on $V$, form the base change $f_{V^{e}}: X_{V^{e}}=X \times_{V} V^{e} \rightarrow V^{e}$, and define $F_{X^{e} / V^{e}}^{e}: X^{e} \rightarrow X_{V^{e}}$ to be the $e$-iterated relative Frobenius morphism. Furthermore, we often assume that $V$ is $F$-finite, in which case we automatically assume that the Frobenius morphism $F_{V}: V \rightarrow V$ satisfies the identity ${ }^{6}\left(F_{V}\right)^{!} \omega_{V}^{\cdot} \simeq_{\text {qis }} \omega_{V}^{\cdot}$. If we say that $V$ is a variety, it is always of finite type over a perfect field $k$.

\footnotetext{
${ }^{5}$ By geometrically reduced, we mean that $X_{T}=X \times_{V} T$ is reduced for all $T \rightarrow V$ with $T$ integral.

${ }^{6}$ For some discussion of this identity, which always holds for varieties or schemes of essentially finite type over a local ring with a dualizing complex, see [BSTZ10, Section 2].
} 


\section{$F$-SINGULARITIES IN FAMILIES}

Because we will be considering numerous different sheaves on the same topological space $X=X^{e}=X \times_{V} V^{e}=$ etc. but with respect to different schemes, we will adopt the following somewhat non-standard notation. We use this because writing numerous operations $\left(F_{X^{e} / V^{e}}^{e}\right)_{*}$ and $\left(F_{X^{e} / V^{e}}^{e}\right)^{-1}$ that do not affect the underlying space is confusing. We would need notation for projections of the form $X^{e} \times_{V^{e}} V^{e+d} \rightarrow V^{e+d}, X^{e}$ in as well as more general relative Frobenius morphisms $F_{\left(X^{e}\right)_{V^{e+d}} / V^{e+d}}:\left(X^{e}\right)_{V^{e+d}}:=X^{e} \times_{V^{e}} V^{e+d} \rightarrow X_{V^{e+d}}$, and maps $X^{e} \times_{V^{e+d+c}} \rightarrow$ $X^{e} \times_{V^{e+d}}$. By using simply modules, this becomes more transparent.

(1) We will use $R$ to denote $\mathscr{O}_{X}$.

(2) We will use $A$ to denote $f^{-1} \mathscr{O}_{V}$.

(3) We will use $A^{1 / p^{e}}$ to denote $f^{-1}\left(F_{V}^{e}\right)_{*} \mathscr{O}_{V}$. We note that this is not an abuse of notation since $V$ is integral.

(4) We will use $R^{1 / p^{e}}$ to denote $\left(F_{X}^{e}\right)_{*} R$. This is a slight abuse of notation if $X$ is not reduced.

(5) We will use $R_{A^{1 / p^{e}}}$ to denote $R \otimes_{A} A^{1 / p^{e}}$.

(6) We will use $\left(R_{A^{1 / p^{e}}}\right)^{1 / p^{d}}$ to denote $R^{1 / p^{d}} \otimes_{A^{1 / p^{d}}} A^{1 / p^{e+d}}$. This may be a slight abuse of notation if $X_{V}$ is not reduced.

(7) Given an $R$-module $M$, we will use $M^{1 / p^{e}}$ to denote $F_{*}^{e} M$. This will generally not cause any confusion because, typically, $M$ will be locally free or even a line bundle.

(8) We use $\omega_{R}$ to denote $\omega_{X}$ and $\omega_{A}$ to denote $f^{-1} \omega_{V}$.

(9) Etc.

Some of the main results of the paper concern restriction to fibers. These statements pertain only to a special set of fibers of $f$, the fibers over perfect points (see the definition below). For example, if $V$ is a curve over an algebraically closed field, then we often restrict to fibers over all closed points and over the perfect closure of the generic point of $V$, but not over the generic point itself.

Definition 2.2 (Perfect points). A perfect point of $V$, for $s \in V$, is a morphism $s=\operatorname{Spec} k(s) \rightarrow$ $V$ from the spectrum of a perfect field $k(s)$ to $V$. It can also be viewed as a choice of a point $v \in V$ and a field extension $k(s)=K \supseteq k(v)$ such that $K$ is perfect. Finally, a neighborhood of a perfect point $s \in V$ is simply a neighborhood of the image $v$ of $s$.

The fact that $f: X \rightarrow V$ is of finite type implies that the relative Frobenius morphism is a finite map. In some cases, this will allow us to avoid assuming that $V$ is $F$-finite.

LEMMA 2.3. With notation as above, since $f: X \rightarrow V$ is of finite type, the relative Frobenius map $f: X^{e} \rightarrow X_{V}$ is a finite map.

Proof. We work locally on $V$ and $X$. It is sufficient to show that $R^{1 / p^{e}}$ is a finite $R_{A^{1 / p^{e}} \text {-module. }}$ Write $S=A\left[x_{1}, \ldots, x_{n}\right]$ and $R=S / I$. We first observe that $S_{A^{1 / p^{e}}} \rightarrow S^{1 / p^{e}}$ is a finite map. However, the $x_{1}^{i_{1}} \cdots x_{n}^{i_{n}}$ for $0 \leqslant i_{j}<p^{e}$ clearly form a generating set for $S^{1 / p^{e}}$ over $S_{A^{1 / p^{e}}}$.

Now, tensor the map $S_{A^{1 / p^{e}}} \rightarrow S^{1 / p^{e}}$ with $\otimes_{S}(S / I)=\otimes_{S} R$. We obtain

$$
R_{A^{1 / p^{e}}}=R \otimes_{A} A^{1 / p^{e}}=(S / I) \otimes_{S} S_{A^{1 / p^{e}}} \rightarrow(S / I) \otimes_{S} S^{1 / p^{e}}=\left(S / I^{\left[p^{e}\right]}\right)^{1 / p^{e}} \rightarrow(S / I)^{1 / p^{e}},
$$

where the final map is the canonical surjection of rings. The map is finite since each part is.

We also recall the result of Radu and André. 


\section{Z. Patakfalvi, K. Schwede And W. Zhang}

Theorem $2.4([\operatorname{Rad} 92$, And93]). Suppose that $f: X \rightarrow V$ is a flat map of Noetherian schemes. Then $f$ has geometrically regular fibers if and only if the relative Frobenius $R_{A^{1 / p^{e}}} \cong A^{1 / p^{e}} \otimes_{A} R$ $\rightarrow R^{1 / p^{e}}$ is flat.

We immediately obtain the following corollary, which will also be useful.

Corollary 2.5. Suppose that $f: X \rightarrow V$ is as in Notation 2.1 and additionally has geometrically regular fibers. Then for any $R_{A^{1 / p^{e}}}$-module $M$, the natural evaluation-at-1 map $\mathscr{H} \mathrm{om}_{R_{A^{1 / p^{e}}}}\left(R^{1 / p^{e}}, M\right) \rightarrow M$ surjects.

Proof. The module $R^{1 / p^{e}}$ is a locally free $R_{A^{1 / p^{e}}}$-module, and the result follows.

Now, we state the object which we will study for the majority of the paper.

Definition 2.6 $(\phi)$. From here on, we fix a line bundle $L$ on $X \cong X^{e}$ and we also fix a (possibly

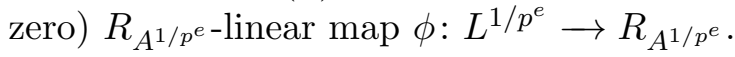

Remark 2.7 (Reflexive sheaves for G1 and S2 morphisms). Suppose that $f: X \rightarrow V$ is G1 and S2. Note that there exists an open set $\iota: U \hookrightarrow X$ such that $X \backslash U$ has codimension at least 2 along each fiber and that $\left.f\right|_{U}$ is a Gorenstein morphism.

Finally, suppose that $M$ is any rank 1 reflexive $R$-module which is locally free on a set $U$ as above. Then $M^{1 / p^{e}}$ is not only reflexive as an $R^{1 / p^{e}}$-module, we claim that it is also reflexive as an $R_{A^{1 / p^{e}}}$-module. Since we already have $\left.i_{*} M\right|_{U}=M$ by Proposition A.7 because $M$ is reflexive, it is sufficient to replace $X$ by $U$. Thus, $\omega_{X / V}$ and $M$ are both locally free as $R$-modules and $\omega_{X_{V}}$ is locally free as an $R_{A^{1 / p^{e}}}$-module. We work locally so as to trivialize all these modules. Then

$$
\begin{aligned}
M^{1 / p^{e}} & \cong \omega_{X / V}^{1 / p^{e}}=\left(F_{X^{e} / V^{e}}^{e}\right)_{*} \omega_{X / V} \\
& \cong \mathscr{H} \operatorname{om}_{\mathscr{O}_{X_{V}}}\left(\left(F_{X^{e} / V^{e}}^{e}\right)_{*} \mathscr{O}_{X}, \omega_{X_{V}^{e} / V^{e}}\right) \\
& \cong \mathscr{H} \operatorname{om}_{R_{A^{1 / p^{e}}}}\left(R^{1 / p^{e}}, R_{A^{1 / p^{e}}}\right),
\end{aligned}
$$

which is clearly reflexive (the second isomorphism follows from Grothendieck duality for the finite relative Frobenius map).

Conversely, if $M$ is any $R$-module which is locally free on a set $U$ and reflexive as an $R_{A^{1 / p^{e}} \text { - }}$ module, then it is also reflexive as an $R^{1 / p^{e}}$-module. To see this, note that $\left.M\right|_{U}$ is reflexive as an

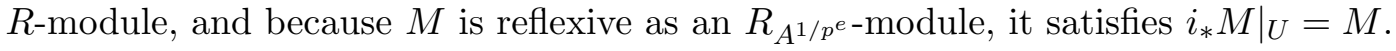

Definition 2.8 ( $\phi$ versus divisors). Observe the following identifications:

$$
\begin{aligned}
& \mathscr{H} \operatorname{om}_{R_{A^{1 / p^{e}}}}\left(L^{1 / p^{e}}, R_{A^{1 / p^{e}}}\right) \\
& \cong \underbrace{\left(\mathscr{H} \operatorname{om}_{R_{A^{1 / p^{e}}}}\left(L^{1 / p^{e}} \otimes_{R_{A^{1 / p^{e}}}}\left(\omega_{R_{A^{1 / p^{e}}} / A^{1 / p^{e}}}\right), \omega_{R_{A^{1 / p^{e}}} / A^{1 / p^{e}}}\right)\right)^{* *}} \\
& \text { (This clearly holds on } U \text {, note that both sheaves are reflexive and use Corollary A.8.) } \\
& =\underbrace{\left(\mathscr{H} \operatorname{om}_{\mathscr{O}_{X_{V^{e}}}}\left(\left(F_{X^{e} / V^{e}}^{e}\right)_{*}\left(L \otimes\left(\left(F_{X^{e} / V^{e}}^{e}\right)^{*} \omega_{X_{V^{e}} / V^{e}}\right)\right), \omega_{X_{V^{e}} / V^{e}}\right)\right)^{* *}} \\
& \text { (This just rewrites the previous line using different notation.) } \\
& \cong \underbrace{\left(\left(F_{X^{e} / V^{e}}^{e}\right)_{*} \mathscr{H} \operatorname{om}_{\mathscr{O}_{X^{e}}}\left(L \otimes\left(\left(F_{X^{e} / V^{e}}^{e}\right)^{*} \omega_{X_{V^{e}} / V^{e}}\right), \omega_{X^{e} / V^{e}}\right)\right)^{* *}}_{\text {(Grothendieck duality for a finite map) }}
\end{aligned}
$$




\section{$F$-SINGULARITIES IN FAMILIES}

$$
\cong \quad \underbrace{\left(F_{X^{e} / V^{e}}^{e}\right)_{*}\left(L^{-1} \otimes \omega_{X^{e} / V^{e}} \otimes\left(F_{X^{e} / V^{e}}^{e}\right)^{*} \omega_{X_{V^{e}} / V^{e}}^{-1}\right)^{* *}} .
$$

(By Remark 2.7 and Corollary A.8, we may take the reflexive hull as $\left(F_{X^{e} / V^{e}}^{e}\right)_{*} \mathscr{O}_{X^{e}}$-modules.)

Now, observe that $\omega_{X / V}$ is compatible with base change up to reflexification. In particular, if our base change is the Frobenius morphism $F_{V}^{e}: V^{e} \rightarrow V$, then writing $\pi_{V^{e}}: X_{V^{e}}=X \times_{V} V^{e} \rightarrow X$ as the projection, we have $\omega_{X_{V} e / V^{e}} \cong\left(\pi_{V^{e}}^{*} \omega_{X / V}\right)^{* *}$ since both sheaves are reflexive and they certainly agree outside the non-relatively Cohen-Macaulay locus (which is of relative codimension at least 2) by Corollary A.8. In particular,

$$
\left.\left.\left(\left(F_{X^{e} / V^{e}}^{e}\right)^{*} \omega_{X_{V^{e}} / V^{e}}\right)^{* *} \cong\left(\left(F_{X^{e} / V^{e}}^{e}\right)^{*} \pi_{V^{e}}^{*} \omega_{X / V}\right)\right)^{* *}=\left(\left(F_{X}^{e}\right)^{*} \omega_{X / V}\right)\right)^{* *}=\left(\omega_{X / V}^{p^{e}}\right)^{* *}
$$

Plugging this into (2.1), we obtain

$$
\mathscr{H} \operatorname{om}_{R_{A^{1 / p^{e}}}}\left(L^{1 / p^{e}}, R_{A^{1 / p^{e}}}\right) \cong\left(F_{X^{e} / V^{e}}^{e}\right)_{*}\left(L^{-1} \otimes \omega_{X^{e} / V^{e}}^{1-p^{e}}\right)^{* *} .
$$

If, additionally, $X$ is absolutely (instead of relatively) G1 and S2 (for example, if $V$ is regular), then any choice of non-degenerate ${ }^{7} \phi$ induces a non-zero, effective Weil divisorial sheaf $D_{\phi}$ such that $\mathscr{O}_{X}\left(D_{\phi}\right) \cong\left(L^{-1} \otimes \omega_{X^{e}}^{1-V^{e}}\right)^{* *}$ by [Har94]. We would like to generalize this to the case that $X \rightarrow V$ is relatively $\mathrm{G} 1$ and $\mathrm{S} 2$.

DeFinition 2.9. We say that $\phi$ is relatively divisorial if $\phi$ locally generates $\mathscr{H} \mathrm{om}_{R_{A^{1 / p^{e}}}}\left(L^{1 / p^{e}}\right.$, $\left.R_{A^{1 / p^{e}}}\right)$ as an $R^{1 / p^{e}}$-module

(a) at the generic points of each fiber and

(b) at the generic point of every codimension 1 singular point of every geometric fiber.

In this case, by removing a set of relative codimension 2 so that $f$ is relatively Gorenstein, we see that $\phi \cdot R^{1 / p^{e}} \subseteq \mathscr{H} \mathrm{om}_{R_{A^{1 / p^{e}}}}\left(L^{1 / p^{e}}, R_{A^{1 / p^{e}}}\right)$ is a rank 1 free submodule of an invertible $R^{1 / p^{e}}$-module. To be able to associate a divisor to this submodule in a sensible way, we should show that it is the trivial (full) submodule at every singular codimension 1 point. Indeed, let $\xi$ be a singular codimension 1 point. Then one of the following cases holds:

- $f(\xi)$ is a codimension 1 point. In this case, $\xi$ is a general point of the fibers over $f(\xi)$, hence assumption (a) guarantees that $\phi$ generates a full submodule at $\xi$.

$\circ f(\xi)$ is the general point of $V$. In this case, $\xi$ is a codimension 1 point of the fiber over $f(\xi)$, and it is not in the smooth locus of $f$. Therefore, assumption (b) shows that again $\phi$ generates a full submodule at $\xi$.

Therefore, the submodule $\phi \cdot R^{1 / p^{e}}$ determines a Cartier divisor and also an honest Weil divisor on the original $X$. We denote this divisor by $D_{\phi}$ as well.

Definition 2.10 ( $\phi$ as a divisor). If $\phi$ is relatively divisorial, we set $\Delta_{\phi}$ to be the $\mathbb{Q}$-divisor $\left(1 /\left(p^{e}-1\right)\right) D_{\phi}$. This makes sense because $D_{\phi}$ is trivial along the codimension 1 components of the singular locus of $X$ and so we avoid the pathological issues which occur for $\mathbb{Q}$-divisors on non-normal spaces.

We now explain how to recover $\phi$ from a $\mathbb{Q}$-divisor.

Remark 2.11 (Obtaining $\phi$ from divisors). We work under the conventions of Definition 2.10. Untangling Definition 2.8 yields a method to obtain $\phi$ from a divisor $\Delta \geqslant 0$ (which then coincides with $\Delta_{\phi}$ ) under the following assumptions:

\footnotetext{
${ }^{7}$ Here, non-degenerate means non-zero on any irreducible component.
} 


\section{Z. Patakfalvi, K. Schwede and W. Zhang}

(a) We have $\Delta=(1 / m) D$ for some Weil divisor $D$, where $p \nmid m$.

(b) The Weil divisor $D$ on $X$ is Cartier in relative codimension 1 .

(c) The support of $D$ does not contain any generic point or any singular codimension 1 point of any geometric fiber.

(d) We have $\left(p^{e}-1\right) / m \in \mathbb{Z}$ and $\left(\omega_{X / V}^{1-p^{e}} \otimes \mathscr{O}_{X}\left(\left(1-p^{e}\right) \Delta\right)\right)^{* *}=L$ is a line bundle.

In such a case, the integral divisor $\left(p^{e}-1\right) \Delta$ (which is well defined since Supp $\Delta$ does not contain the codimension 1 components of the non-regular locus of $X$, cf. [KAAC92, pp. 171-173] or [MS12, Section 2.2]) induces an inclusion

$$
\left(F_{X^{e} / V^{e}}^{e}\right)_{*} L \hookrightarrow\left(F_{X^{e} / V^{e}}^{e}\right)_{*}\left(\left(\omega_{X / V}^{1-p^{e}}\right)^{* *}\right) .
$$

This composed with the natural Grothendieck trace map

$$
\left(F_{X^{e} / V^{e}}^{e}\right)_{*}\left(\left(\omega_{X / V}^{1-p^{e}}\right)^{* *}\right) \rightarrow \mathscr{O}_{X_{V}}
$$

yields a map $\phi$. It is easy to see that conditions (a)-(d) above guarantee that $\phi$ is relatively divisorial. Furthermore, we also have $\Delta=\Delta_{\phi}$.

For future reference we make the following definition.

DeFinition 2.12. In the situation of Notation 2.1, $(X, \Delta)$ is a pair if $f$ is $\mathrm{G} 1$ and $\mathrm{S} 2$ and $\Delta$ satisfies the assumptions of Remark 2.11.

\subsection{Composing maps}

Given $\phi$ as in Definition 2.8, we can compose $\phi: L^{1 / p^{e}} \rightarrow R_{A^{1 / p^{e}}}$ with itself (after twisting), similarly to [BS13, Section 4] or [Sch09], and thus obtain new maps

$$
\phi^{2} \in \operatorname{Hom}_{R_{A^{1 / p^{2 e}}}}\left(\left(L^{\left(p^{e}+1\right)}\right)^{1 / p^{2 e}}, R_{A^{1 / p^{2 e}}}\right)
$$

and, more generally,

$$
\phi^{n} \in \operatorname{Hom}_{R_{A^{1 / p^{n e}}}}\left(\left(L^{\frac{p^{n e}-1}{p^{e}-1}}\right)^{1 / p^{n e}}, R_{A^{1 / p^{n e}}}\right) .
$$

We explain this construction.

Begin by tensoring $\phi$ by $L$ over $R$, and then taking $1 / p^{e}$ th roots; we obtain

$$
\left(L^{1+p^{e}}\right)^{1 / p^{2 e}}=\left(L^{1 / p^{e}} \otimes_{R} L\right)^{1 / p^{e}} \rightarrow\left(\left(R \otimes_{A} A^{1 / p^{e}}\right) \otimes_{R} L\right)^{1 / p^{e}}=L^{1 / p^{e}} \otimes_{A^{1 / p^{e}}} A^{1 / p^{2 e}} .
$$

On the other hand, we can also tensor $\phi$ by $A^{1 / p^{2 e}}$ over $A^{1 / p^{e}}$ to obtain

$$
L^{1 / p^{e}} \otimes_{A^{1 / p^{e}}} A^{1 / p^{2 e}} \rightarrow R_{A^{1 / p^{2 e}}} .
$$

By composing (2.3) and (2.4), we obtain the desired map $\phi^{2}$. We now define $\phi^{n}$ inductively as follows. Given

$$
\phi^{n-1}:\left(L^{\frac{p^{(n-1) e}-1}{p^{e}-1}}\right)^{1 / p^{(n-1) e}} \rightarrow R_{A^{1 / p}(n-1) e}
$$




\section{$F$-SINGULARITIES IN FAMILIES}

tensor with $L$ over $R$ and then take $p^{e}$ th roots, which yields

$$
\begin{aligned}
\left(L^{\frac{p^{n e}-1}{p^{e}-1}}\right)^{1 / p^{n e}} & =\left(\left(L^{\frac{p^{(n-1) e}-1}{p^{e}-1}+p^{(n-1) e}}\right)^{1 / p^{(n-1) e}}\right)^{1 / p^{e}} \\
& =\left(L \otimes_{R}\left(L^{\frac{p^{(n-1) e}-1}{p^{e}-1}}\right)^{1 / p^{(n-1) e}}\right)^{1 / p^{e}} \\
& \rightarrow\left(L \otimes_{R} R_{A^{1 / p^{(n-1) e}}}\right)^{1 / p^{e}}=L^{1 / p^{e}} \otimes_{A^{1 / p^{e}}} A^{1 / p^{n e}}
\end{aligned}
$$

We then apply $\phi$ to the first term in the final tensor product to obtain

$$
\left(L^{\frac{p^{n e}-1}{p^{e}-1}}\right)^{1 / p^{n e}} \rightarrow L^{1 / p^{e}} \otimes_{A^{1 / p^{e}}} A^{1 / p^{n e}} \stackrel{\phi \otimes \cdots}{\longrightarrow}\left(R \otimes_{A} A^{1 / p^{e}}\right) \otimes_{A^{1 / p^{e}}} A^{1 / p^{n e}} \cong R_{A^{1 / p^{n e}}},
$$

which we take as the official definition of $\phi^{n}$. On the other hand, for every $0<m<n$, one can look at the following composition of $\phi^{m}$ and $\phi^{n-m}$ :

$$
\begin{aligned}
\gamma:\left(L^{\frac{p^{n e}-1}{p^{e}-1}}\right)^{1 / p^{n e}} & \cong\left(L^{\frac{p^{m e}-1}{p^{e}-1}} \otimes_{R} L^{\frac{p^{n e}-p^{m e}}{p^{e}-1}}\right)^{1 / p^{n e}} \cong\left(\left(L^{\frac{p^{m e}-1}{p^{e}-1}}\right)^{1 / p^{m e}} \otimes_{R} L^{\frac{p^{(n-m) e}-1}{p^{e}-1}}\right)^{1 / p^{(n-m) e}} \\
& \stackrel{\left(\phi^{m} \otimes \mathrm{id}_{L} \cdots\right)^{1 / p^{(n-m) e}}}{\longrightarrow}\left(\left(R \otimes_{A} A^{\frac{1}{p^{m e}}}\right) \otimes_{R} L^{\frac{p^{(n-m) e}-1}{p^{e}-1}}\right)^{1 / p^{(n-m) e}} \\
& \cong\left(L^{\frac{p^{(n-m) e}-1}{p^{e}-1}}\right)^{1 / p^{(n-m) e}} \otimes_{A^{1 / p^{(n-m) e}}} A^{1 / p^{n e}} \\
& \stackrel{\phi^{n-m} \otimes_{A^{\cdots} \cdots} \cdots}{\longrightarrow}\left(R \otimes_{A} A^{1 / p^{(n-m) e}}\right) \otimes_{A^{1 / p^{(n-m) e}}} A^{1 / p^{n e}} \cong R \otimes_{A} A^{1 / p^{n e}}
\end{aligned}
$$

In particular, taking $m=1$ gives an a priori different map which we could also define as $\phi^{n}$. We now explain why this map is actually equal to the official $\phi^{n}$.

LEMMA 2.13. With notation as above, $\gamma=\phi^{n}$.

Proof. The statement is local, and so we may suppose $L=R$. With the (obscuring) powers of $L$ removed, $\phi^{n}$ is described as the following composition:

$$
\begin{aligned}
R^{1 / p^{n e}} & \stackrel{\phi^{1 / p^{(n-1) e}}}{\longrightarrow} R^{1 / p^{(n-1) e}} \otimes_{A^{1 / p^{(n-1) e}}} A^{1 / p^{n e}} \\
& \stackrel{\phi^{1 / p^{(n-2) e}} \otimes \cdots}{\longrightarrow}\left(R^{1 / p^{(n-2) e}} \otimes_{A^{1 / p^{(n-2) e}}} A^{1 / p^{(n-1) e}}\right) \otimes_{A^{1 / p^{(n-1) e}}} A^{1 / p^{n e}} \\
& \stackrel{\phi^{1 / p^{(n-3) e}} \otimes \cdots}{\longrightarrow} \cdots \cdots \cdots \\
\vdots & \stackrel{\phi^{1 / p^{e}} \otimes \cdots}{\longrightarrow} R^{1 / p^{e}} \otimes_{A^{1 / p^{e}}} \cdots \otimes_{A^{1 / p^{(n-1) e}}} A^{1 / p^{n e}} \\
& \stackrel{\phi \otimes \cdots}{\longrightarrow} R \otimes_{A} \cdots \otimes_{A^{1 / p^{(n-1) e}}} A^{1 / p^{n e}} .
\end{aligned}
$$

The first $m$ entries in the composition make up $\left(\phi^{m} \otimes \cdots\right)^{1 / p^{(n-m) e}}$ in $(2.5)$, and the last $n-m$ entries clearly yield $\phi^{n-m} \otimes_{A^{1 / p}(n-m) e} A^{1 / p^{n}}$, as desired. The result is then obvious.

LEMma 2.14. With notation as in Definition 2.8, assume additionally that $\phi$ is relatively divisorial; then $\phi^{n}$ is relatively divisorial and $\Delta_{\phi}=\Delta_{\phi^{n}}$ for every integer $n \geqslant 1$.

Proof. For showing any of the two statements, we may remove the non-relatively Gorenstein locus. That is, by possibly further restricting $R$, we may assume that $R$ is relatively Gorenstein 


\section{Z. Patakfalvi, K. Schwede and W. Zhang}

over $V$ and that $L$ is trivial (since our map $f: X \rightarrow V$ is geometrically G1 and S2). Hence, by Remark 2.7,

$$
\operatorname{Hom}_{R \otimes_{A} A^{1 / p^{i e}}}\left(R^{1 / p^{i e}}, R \otimes_{A} A^{1 / p^{i e}}\right) \cong R^{1 / p^{i e}} \quad \text { for all } i .
$$

From [Kun86, Appendix F], [Sch09, Lemma 3.9], or composition of Grothendieck trace, we have

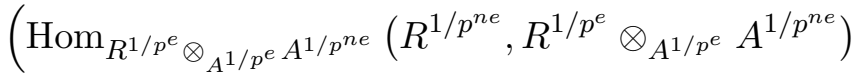

$$
\begin{aligned}
& \left.\otimes_{R^{1 / p^{e}} \otimes_{A^{1 / p}}} A^{1 / p^{n e}} \operatorname{Hom}_{A_{A^{1 / p^{n e}}}}\left(R^{1 / p^{e}} \otimes_{A^{1 / p^{e}}} A^{1 / p^{n e}}, R_{A^{1 / p^{n e}}}\right)\right) \\
& \cong \operatorname{Hom}_{R_{A^{1 / p}} \text { ne }}\left(R^{1 / p^{n e}}, R_{A^{1 / p^{n e}}}\right)
\end{aligned}
$$

via the homomorphism induced by composition. Let $\theta_{e}$ be the $R^{1 / p^{e}}$-module generator of

$$
\operatorname{Hom}_{R \otimes_{A} A^{1 / p^{e}}}\left(R^{1 / p^{e}}, R \otimes_{A} A^{1 / p^{e}}\right)
$$

for each $e$, and let $\theta_{e}^{n}$ be defined as $\varphi^{n}$, with $\varphi$ replaced by $\theta$. Then by using (2.6) iteratively, $\theta_{e}^{n}=\theta_{n e}$ up to multiplication by a unit.

Further, let $r \in R$ be such that $\varphi\left(\_\right)=\theta_{e}\left(r^{1 / p^{e}}{ }_{-}\right)$. Then it is easy to verify that

$$
\varphi^{n}\left(\_\right)=\theta_{i e}\left(\left(r^{\frac{p^{n e}-1}{p^{e}-1}}\right)^{1 / p^{n e}} \cdot-\right) \text {. }
$$

Then we see that if $\phi$ is generating at a point $P \in X$, or equivalently $r$ is a unit at $P$, then so is $\phi^{n}$. This shows that $\phi^{n}$ is relatively divisorial. Furthermore, (2.7) shows that $\Delta_{\phi^{i}}=\Delta_{\phi}$.

\subsection{Base change of $\phi$}

Suppose that we are given a morphism of schemes $g: T \rightarrow V$ such that $T$ is also excellent and integral and has a dualizing complex. For example, we could set $T$ to be a closed point of $V$ and let $g$ be the inclusion. Alternately, we could let $g$ be a regular alteration over some closed subscheme of $V$. We list the following maps:

$$
\begin{array}{rlll}
p_{1}: & X \times_{V} T & \rightarrow X & \text { (the projection) }, \\
\left(p_{1}\right)^{1 / p^{i}}: & X^{i} \times_{V^{i}} T^{i} & \rightarrow X^{i} & \text { (the projection for any } i), \\
q_{i}: & X \times_{V} T^{i} & \rightarrow X \times_{V} V^{i} & \text { (base change) }, \\
p_{1}=q_{0}: & X \times_{V} T & \rightarrow X . &
\end{array}
$$

These are pictured below:

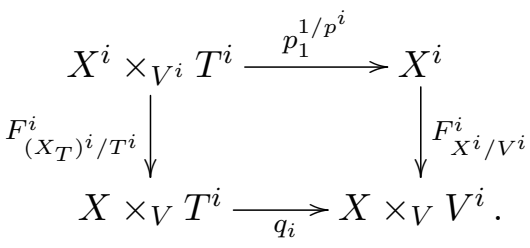

Notice that given $\phi: L^{1 / p^{e}} \rightarrow R_{A^{1 / p^{e}}}=\mathscr{O}_{X_{V} e}$, we can form $\left(q_{e}\right)^{*} \phi$, which we denote by

$$
\phi_{T}: L_{T}^{1 / p^{e}} \cong q_{e}^{*} L^{1 / p^{e}} \rightarrow q_{e}^{*} \mathscr{O}_{X_{V^{e}}}=\mathscr{O}_{X_{T}} .
$$

We explain the isomorphism $L_{T}^{1 / p^{e}} \cong q_{e}^{*} L^{1 / p^{e}}$ briefly. Working locally, let $V=\operatorname{Spec} A, T=$ $\operatorname{Spec} B$, and $X=\operatorname{Spec} R$. Then the map $\phi_{T}$ is identified with the map

$$
L^{1 / p^{e}} \otimes_{A_{A^{1 / p^{e}}}} R_{B^{1 / p^{e}}} \cong L^{1 / p^{e}} \otimes_{A^{1 / p^{e}}} B^{1 / p^{e}} \rightarrow\left(\left(R \otimes_{A} A^{1 / p^{e}}\right) \otimes_{A^{1 / p^{e}}} B^{1 / p^{e}}\right) \cong R \otimes_{A} B^{1 / p^{e}} .
$$

The isomorphism in the definition of $\phi_{T}$ is now immediate. 


\section{$F$-SINGULARITIES IN FAMILIES}

The next lemma shows that base change of $\phi$ commutes with the self-composition defined in Section 2.1.

Lemma 2.15. Suppose that $g: T \rightarrow V$ is as above. Then $\left(\phi^{n}\right)_{T}=\left(\phi_{T}\right)^{n}$.

Proof. It is sufficient to prove this in the affine case, assuming that our isomorphism is sufficiently canonical (which will be clear). We notice that $\left(\phi_{T}\right)^{2}$ is the composition

$$
\begin{aligned}
\left(\left(L^{p^{e}+1}\right)^{1 / p^{e}} \otimes_{A^{1 / p^{e}}} B^{1 / p^{e}}\right)^{1 / p^{e}} & \stackrel{\left(\phi_{T} \otimes_{R_{B^{1 / p}}} L_{T}\right)^{1 / p^{e}}}{\longrightarrow} L^{1 / p^{e}} \otimes_{A^{1 / p^{e}}} B^{1 / p^{e}} \otimes_{B^{1 / p^{e}}} B^{1 / p^{2 e}} \\
& \stackrel{\phi_{T} \otimes_{B^{1 / p^{e}}} B^{1 / p^{2 e}}}{\longrightarrow}\left(\left(R \otimes_{A} A^{1 / p^{e}}\right) \otimes_{A^{1 / p^{e}}} B^{1 / p^{e}}\right) \otimes_{B^{1 / p^{e}}} B^{1 / p^{2 e}} .
\end{aligned}
$$

Now, unraveling the definitions, we obtain

$$
\begin{aligned}
\left(\phi_{T}\right)^{2} & =\left(\left(\phi_{T} \otimes_{R_{B^{1 / p}} e} L_{T}\right)^{1 / p^{e}}\right) \circ\left(\phi_{T} \otimes_{B^{1 / p^{e}}} B^{1 / p^{2 e}}\right) \\
& \left.=\left(\left(\phi \otimes_{A^{1 / p^{e}}} B^{1 / p^{e}}\right) \otimes_{R_{B^{1 / p^{e}}}}\left(L \otimes_{A^{1 / p^{e}}} B^{1 / p^{e}}\right)\right)^{1 / p^{e}}\right) \circ\left(\phi \otimes_{A^{1 / p^{e}}} B^{1 / p^{e}} \otimes_{B^{1 / p^{e}}} B^{1 / p^{2 e}}\right) \\
& =\left(\left(\phi \otimes_{A_{A^{1 / p^{e}}}} L \otimes_{A^{1 / p^{e}}} B^{1 / p^{e}}\right)^{1 / p^{e}}\right) \circ\left(\phi \otimes_{A^{1 / p^{e}}} B^{1 / p^{2 e}}\right) \\
& =\left(\left(\phi \otimes_{A_{A^{1 / p^{e}}}} L\right)^{1 / p^{e}} \otimes_{A^{1 / p^{2 e}}} B^{1 / p^{2 e}}\right) \circ\left(\left(\phi \otimes_{A^{1 / p^{e}}} A^{1 / p^{2 e}}\right) \otimes_{A^{1 / p^{2 e}}} B^{1 / p^{2 e}}\right) \\
& =\left(\left(\phi \otimes_{R_{A^{1 / p^{e}}}} L\right)^{1 / p^{e}} \circ\left(\phi \otimes_{A^{1 / p^{e}}} A^{1 / p^{2 e}}\right)\right) \otimes_{A^{1 / p^{2 e}}} B^{1 / p^{2 e}} \\
& =\left(\phi^{2}\right)_{T},
\end{aligned}
$$

as desired. The general $n$th self-composition is similar.

Our next goal is to describe how divisors, corresponding to maps $\varphi$, fare under base change. We thank Brian Conrad for pointing us in the right direction [Con00, Theorem 3.61].

Lemma 2.16. Suppose that $f: X \rightarrow V$ is a finite-type, Cohen-Macaulay morphism over an excellent scheme $V$ of characteristic $p>0$ and that $g: T \rightarrow V$ is as above. Then the Grothendieck trace map

$$
\left(F_{X^{e} / V^{e}}\right)_{*} \omega_{X^{e} / V^{e}} \cong \mathscr{H} \operatorname{om}_{\mathscr{O}_{X_{V}}}\left(\left(F_{X^{e} / V^{e}}\right)_{*} \mathscr{O}_{X^{e}}, \omega_{X_{V^{e}} / V^{e}}\right) \rightarrow \omega_{X_{V^{e}} / V^{e}}
$$

is compatible with base change.

Proof. We clearly have a commutative diagram:

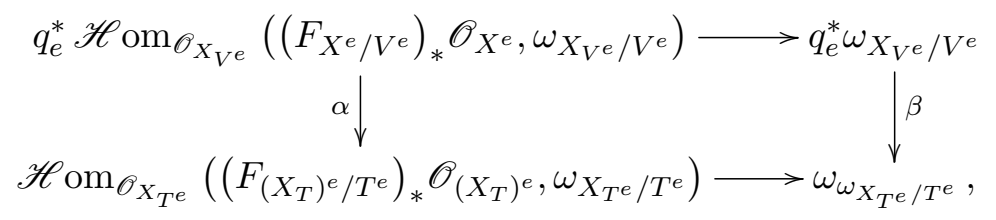

and by [Con00, Theorem 3.6.1], the map $\beta$ is an isomorphism. It is sufficient to verify that $\alpha$ is an isomorphism as well. We work locally on some affine chart on $X$ and hence assume that $X_{V} \subseteq \mathbb{A}_{V}^{N}=: P_{V}$ embeds as a closed subscheme since $f$ is of finite type. The map $\alpha$ can then be identified with

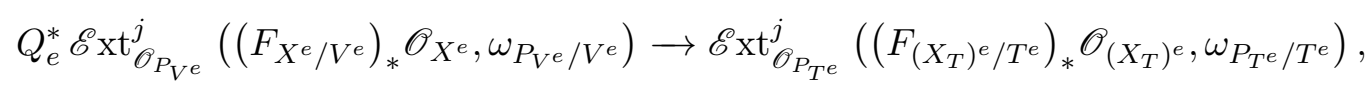

where $Q_{e}: P_{T^{e}} \rightarrow P_{V^{e}}$ is the induced map and $j=N-\operatorname{dim}(X / V)$ (we leave off the pushforward for the inclusion $i: X_{V} \rightarrow P_{V}$ above). This in turn can be identified with

$$
Q_{e}^{*} \mathscr{E} \mathrm{xt}_{\mathscr{O}_{\left(P_{V}\right)^{e}}}^{j}\left(\mathscr{O}_{X^{e}}, \omega_{\left(P_{V}\right)^{e} / V^{e}}\right) \rightarrow \mathscr{E}^{\mathrm{xt}_{\mathscr{O}_{\left(P_{T}\right)^{e}}}^{j}}\left(\mathscr{O}_{\left(X_{T}\right)^{e}}, \omega_{\left(P_{T}\right)^{e} / T^{e}}\right)
$$




\section{Z. Patakfalvi, K. Schwede And W. Zhang}

This last map is exactly the bottom row of [Con00, Diagram (3.6.1) in Theorem 3.6.1], which is an isomorphism, and hence the proof is complete.

Lemma 2.16 above allows us to show that if the divisor $D_{\phi}$ or $\Delta_{\phi}$ is trivial, then it stays trivial after base change.

Lemma 2.17 (Divisors which are zero stay zero). Suppose that $f: X \rightarrow V$ is a $G 1$ and S2 morphism and that $\phi: L^{1 / p^{e}} \rightarrow R_{A^{1 / p^{e}}}$ is as above. Additionally, suppose that $g: T \rightarrow V$ is any base change. Finally, suppose that the natural map

$$
\phi \cdot R^{1 / p^{e}} \rightarrow \mathscr{H} \operatorname{om}_{R_{A^{1 / p^{e}}}}\left(L^{1 / p^{e}}, R_{A^{1 / p^{e}}}\right) \cong\left(F_{X^{e} / V^{e}}^{e}\right)_{*}\left(L^{-1} \otimes \omega_{X^{e} / V^{e}}^{1-p^{e}}\right)^{* *}
$$

is an isomorphism of $R^{1 / p^{e}}$-modules (here, we define $\phi \cdot R^{1 / p^{e}}$ to be the $R^{1 / p^{e}}$-submodule of the set $\mathscr{H} \operatorname{om}_{R_{A^{1 / p}}}\left(L^{1 / p^{e}}, R_{A^{1 / p^{e}}}\right)$ generated by $\left.\phi\right)$. Then

$$
\phi_{T} \cdot\left(R_{T}\right)^{1 / p^{e}} \rightarrow \mathscr{H} \operatorname{om}_{R_{T^{1 / p^{e}}}}\left(L_{T}^{1 / p^{e}}, R_{T^{1 / p^{e}}}\right) \cong\left(F_{\left(X_{T}\right)^{e} / T^{e}}^{e}\right)_{*}\left(L_{T}^{-1} \otimes \omega_{\left(X_{T}\right)^{e} / T^{e}}^{1-p^{e}}\right)^{* *}
$$

is also an isomorphism.

In particular, if $\phi$ is relatively divisorial (see Definition 2.9), then the following holds: if $D_{\phi}$ is zero, then so is $D_{\phi_{T}}$.

Proof. The statement about divisors is trivial since it is easy to see that a divisor being zero corresponds to the map above being an isomorphism. Thus, we merely need to prove the assertion. However, since $f$ and all the sheaves involved are relatively S2, it suffices to prove the statement off a set of relative codimension 2 by Corollary A.8. Therefore, by removing a set of codimension 2 , we can assume that $f$ is a Gorenstein morphism. Thus, working locally if needed, $\phi$ can be identified (up to multiplication by a unit in $R^{1 / p^{e}}$ ) with the Grothendieck trace $\left(F_{X^{e}}^{e} / V^{e}\right)_{*} \omega_{X^{e}} / V^{e} \rightarrow$ $\omega_{X_{V} / V^{e}}$. Hence, by Lemma 2.16, so can $\phi_{T}$. The proof is complete.

We now move on to a discussion of base change with respect to divisors. First, we observe that by Lemma 2.17, if $\phi$ is relatively divisorial, then so is $\phi_{T}$ for every base change $g: T \rightarrow V$.

DEFinition 2.18. Suppose that for some relatively divisorial $\phi$, we have $\Delta_{\phi}$ as above and that $g: T \rightarrow V$ is a base change. Then, we write $\Delta_{\phi} \rrbracket_{X_{T}}$ (respectively, $D_{\phi} \rrbracket_{X_{T}}$ ) to denote the divisor $\Delta_{\phi_{T}}$ (respectively, $D_{\phi_{T}}$ ).

Lemma 2.19 (Pulling back $\Delta_{\phi}$ ). Suppose that $f: X \rightarrow V$ is $G 1$ and $S 2$ and that $\phi$ is relatively divisorial. Then for any $g: T \rightarrow V$, we have $\Delta_{\phi} \rrbracket X_{T}=\left(p_{1}^{1 / p^{e}}\right)^{*} \Delta_{\phi}$ (recall the morphism $p_{1}^{1 / p^{e}}: X^{e} \times_{V^{i}} T^{i} \rightarrow X_{i}$ from $\left.(2.8)\right)$.

Here, even though $\Delta_{\phi}$ is not necessarily $\mathbb{Q}$-Cartier, $\left(p_{1}^{1 / p^{e}}\right)^{*} \Delta_{\phi}$ can be defined after removing a set of relative codimension 2 outside of which it is $\mathbb{Q}$-Cartier (since $D_{\phi}$ is Cartier on such a set).

Proof. The statement is local on $X$ and can be checked after removing a relative codimension 2 set, and so we may assume that $D_{\phi}$ is a Cartier divisor. Thus, we assume that $L$ is trivial on the affine scheme $X=\operatorname{Spec} R$ and that $f$ is a Gorenstein morphism. Then, shrinking the neighborhood further if necessary, the map $\phi: R^{1 / p^{e}} \rightarrow R_{A^{1 / p^{e}}}$ can be identified with $s^{1 / p^{e}} \cdot \operatorname{Tr}$, where $\operatorname{Tr}:\left(F_{X^{e} / V^{e}}^{e}\right) * \omega_{X^{e} / V^{e}} \rightarrow \omega_{X_{V}{ }^{e} / V^{e}}$ is the Grothendieck trace. The divisor $D_{\phi}$ is then easily seen to be the $\operatorname{divisor}_{\operatorname{div}_{X}}(s)$. On the other hand, it is clear by Lemma 2.16 that $\phi_{T}$ is then the trace on $X_{T}$ multiplied by $s$ as well. In particular, it equals $\left(p_{1}^{1 / p^{e}}\right)^{*} D_{\phi}$, as desired. 


\section{$F$-SINGULARITIES IN FAMILIES}

Corollary 2.20. Suppose that $g: z \rightarrow V$ is the inclusion of a point. Suppose that $\phi$ is relatively divisorial and corresponds to $\Delta_{\phi}$. Then $\left.\Delta_{\phi}\right|_{X_{z}}=\Delta_{\phi_{z}}$.

Proof. The divisor $\Delta_{\phi_{z}}$ is determined in codimension 1, where $\left.\Delta_{\phi}\right|_{X_{z}}$ and $\Delta_{\phi_{z}}$ agree.

\subsection{Passing to $V^{\infty}$ and other perfect points}

First, suppose that $V$ is the spectrum of a perfect field $A$. Then, the map $L^{1 / p^{e}} \rightarrow R_{A^{1 / p^{e}}}$ is also a map $L^{1 / p^{e}} \rightarrow R$ since $R_{A^{1 / p^{e}}} \cong R$. In such a case, we often also typically write the map as $\psi$ to help distinguish how we are thinking about it. We note that maps such as $\psi: L^{1 / p^{e}} \rightarrow R$ can be composed with themselves as in [BS13, Section 4.1] and, furthermore, this composition $\psi^{n}$ coincides with $\phi^{n}$. For the rest of the subsection, we discuss base change to perfect points. We first consider the perfection of the generic point of $V$.

Set $V^{\infty}$ to denote the not-necessarily-Noetherian scheme $\operatorname{Spec} \mathscr{O}_{V}^{1 / p^{\infty}}$, and set $\eta_{\infty}$ to be the generic point of $V^{\infty}$ with perfect fraction field $k\left(V^{\infty}\right)$ (note that this is a perfect point). We obtain a map $f_{k\left(V^{\infty}\right)}: X_{k\left(V^{\infty}\right)}=X \times_{V} \operatorname{Spec} k\left(V^{\infty}\right) \rightarrow \operatorname{Spec} k\left(V^{\infty}\right)$; now, $X_{k\left(V^{\infty}\right)}$ is a scheme of finite type over a perfect field.

As in Section 2.2, the map $\phi$ then induces a map

$$
\phi_{\infty}: L^{1 / p^{e}} \otimes_{k\left(V^{e}\right)} k\left(V^{\infty}\right) \rightarrow R_{k\left(V^{\infty}\right)}:=R \otimes_{A} k\left(A^{1 / p^{\infty}}\right),
$$

which can be identified with

since $\left(\left(F_{X^{e} / V^{e}}^{e}\right)_{*} \mathscr{O}_{X^{e}}\right) \otimes_{V^{e}} k\left(V^{\infty}\right) \cong F_{*}^{e} \mathscr{O}_{X_{k\left(V^{\infty}\right)}}$.

$$
\psi_{\infty}:\left(L_{k\left(V^{\infty}\right)}\right)^{1 / p^{e}} \rightarrow R_{k\left(V^{\infty}\right)}
$$

More generally, if $s \in V$ is any perfect point, we can induce

$$
\phi_{s}: L_{s}^{1 / p^{e}}:=L^{1 / p^{e}} \otimes_{A^{1 / p^{e}}} k(s)^{1 / p^{e}} \rightarrow R \otimes_{A} k(s)^{1 / p^{e}} .
$$

Since $k(s)=k(s)^{1 / p^{e}}$ is perfect, we can identify this with a $p^{-e}$-linear map

$$
\psi_{s}: L_{s}^{1 / p^{e}} \rightarrow R_{s}
$$

As above, we see that the composition of $\psi_{s}$ in the sense of [BS13, Section 4.1] coincides with the composition $\phi$ as in Section 2.1. Finally, we study this process with respect to divisors.

LEMma 2.21. With notation as above, $\left.\Delta_{\phi}\right|_{X_{s}}=\Delta_{\psi_{s}}$, where $\Delta_{\phi}$ is as in Definition 2.10 and $\Delta_{\psi_{s}}$ is as in [BS13, Section 4].

Proof. By Lemma 2.19, we simply must show that $\Delta_{\phi_{s}}$ coincides with $\Delta_{\psi_{s}}$. Working locally and removing a set of relative codimension 2 , we may assume that $\Phi \in \operatorname{Hom}_{R_{s^{1 / p}}}\left(\left(R_{s}\right)^{1 / p^{e}}, R_{s^{1 / p^{e}}}\right)$ generates the group Hom as an $\left(R_{s}\right)^{1 / p^{e}}$-module and that $\phi\left(\_\right)=\Phi\left(z^{s^{1 / p^{e}}}\right.$. $)$. Thus, $\Delta_{\phi}=$ $\left(1 /\left(p^{e}-1\right)\right) \operatorname{div}_{X}(z)$.

We then identify $R_{s^{1 / p}}$ with $R_{s}$ and hence $\phi$ with $\psi$. Likewise, we can identify $\Phi$ with $\Psi$, which now generates $\operatorname{Hom}_{R_{s}}\left(\left(R_{s}\right)^{1 / p^{e}}, R_{s}\right)$ as an $\left(R_{s}\right)^{1 / p^{e}}$-module. Hence $\psi\left(\_\right)=\Psi\left(z^{1 / p^{e}}\right.$. $\left.\_\right)$ and so $\Delta_{\psi}=\left(1 /\left(p^{e}-1\right)\right) \operatorname{div}_{X}(z)$, as desired.

\section{Relative non-F-pure ideals}

With notation as above, by the Hartshorne-Speiser-Lyubeznik-Gabber theorem [Gab04, Lemma 13.1], cf. [HS77, Lyu97, Bli13], we know that the chain

$$
R_{k\left(V^{\infty}\right)} \supseteq \psi_{\infty}\left(\left(L_{k\left(V^{\infty}\right)}\right)^{1 / p^{e}}\right) \supseteq \psi_{\infty}^{2}\left(\left(L_{k\left(V^{\infty}\right)}^{p^{e}+1}\right)^{1 / p^{2 e}}\right) \supseteq \cdots \supseteq \psi_{\infty}^{n}\left(\left(L_{k\left(V^{\infty}\right)}^{\frac{p^{n e}-1}{p^{e}}}\right)^{1 / p^{n e}}\right) \supseteq \cdots
$$




\section{Z. Patakfalvi, K. Schwede And W. Zhang}

eventually stabilizes. Say that it stabilizes at $n \geqslant n_{0}$. For the rest of this section, we fix this integer $n_{0}$ and make the following definition.

DeFinition 3.1. With notation as above, we define the integer $n_{0}$ to be the uniform integer for $\sigma$ over the generic point of $V$; in general, it will be denoted by $n_{\sigma(\phi), k(V)}$. We notice that for any point $\eta \in V$, we can base change Spec $k(\eta) \rightarrow V$ and form a corresponding integer $n_{\sigma\left(\phi_{\eta}\right), k(\eta)}$.

On the other hand, without the passing to $k\left(V^{\infty}\right)$, we have the images

$$
\begin{array}{ll}
\mathfrak{a}_{1}:=\phi^{1}\left(L^{1 / p^{e}}\right) & \subseteq R_{A^{1 / p^{e}}}, \\
\mathfrak{a}_{2}:=\phi^{2}\left(\left(L^{\left(p^{e}+1\right)}\right)^{1 / p^{2 e}}\right) \subseteq R_{A^{1 / p^{2 e}}}, \\
\vdots \\
\mathfrak{a}_{n}:=\phi^{n}\left(\left(L^{\frac{p^{n e}-1}{p^{e}-1}}\right)^{1 / p^{n e}}\right) \subseteq R_{A^{1 / p^{n e}}}, \\
\vdots
\end{array}
$$

These are ideals of different rings. However, we do have the following relation for any $i>j$ :

$$
\operatorname{im}\left(\mathfrak{a}_{j} \otimes_{A^{1 / p^{j}}} A^{1 / p^{i}} \rightarrow R_{A^{1 / p^{i}}}\right) \supseteq \mathfrak{a}_{i} .
$$

This is straightforward, so we leave it to the reader to check (note that the image of (2.4) contains the image of $\phi^{2}$ ). Additionally, observe that if $A$ were regular, $A^{1 / p^{e}}$ would be flat over $A$ by [Kun69], and so we could identify the tensor product $\mathfrak{a}_{j} \otimes_{A^{1 / p^{j}}} A^{1 / p^{i}}$ with its image in $R_{A^{1 / p^{i}}}$. Therefore, for any integer $n \geqslant i$, we set

$$
\mathfrak{a}_{i, n}=\operatorname{im}\left(\mathfrak{a}_{i} \otimes_{A^{1 / p^{i}}} A^{1 / p^{n}} \rightarrow R_{A^{1 / p^{n}}}\right)
$$

and consider the chain of ideals

$$
R_{A^{1 / p^{n e}}} \supseteq \mathfrak{a}_{1, n} \supseteq \mathfrak{a}_{2, n} \supseteq \cdots \supseteq \mathfrak{a}_{n-1, n} \supseteq \mathfrak{a}_{n, n} .
$$

DEFinition 3.2 ( $n$th relative non- $F$-pure ideal). For every integer $n \geqslant n_{0}=n_{\sigma(\phi), k(V)}$, we define the $n$th limiting relative non-F-pure ideal to be $\mathfrak{a}_{n, n}=\mathfrak{a}_{n}$. It is denoted by $\sigma_{n}(X / V, \phi)$.

We now obtain a relative version of the Hartshorne-Speiser-Lyubeznik-Gabber theorem.

Proposition 3.3. Fix the notation as above. Then there exists a non-empty open subset $U \subseteq V$ of the base scheme $V$ satisfying the following for every integer $m \geqslant n \geqslant n_{0}$ :

$$
\left.\sigma_{m}(X / V, \phi)\right|_{f^{-1}(U)}=\left.\operatorname{im}\left(\sigma_{n}(X / V, \phi) \otimes_{A^{1 / p^{n e}}} A^{1 / p^{m e}} \rightarrow R_{A^{1 / p^{m e}}}\right)\right|_{f^{-1}(U)} .
$$

Proof. For any $m \geqslant n$, we consider the containment $\mathfrak{a}_{n, m} \supseteq \mathfrak{a}_{m, m}$. Fix $k(V)$ to be the residue field of the generic point $\eta \in V$, and consider the induced containment

$$
\mathfrak{a}_{n, m} \otimes_{A} k(V)=\mathfrak{a}_{n, m} \otimes_{A^{1 / p^{m}}} k(V)^{1 / p^{m}} \supseteq \mathfrak{a}_{m, m} \otimes_{A^{1 / p^{m}}} k(V)^{1 / p^{m}}=\mathfrak{a}_{m, m} \otimes_{A} k(V),
$$

since inverting an element is the same as inverting its $p$ th power. We notice two identifications

$$
\begin{gathered}
\mathfrak{a}_{n, m} \otimes_{A^{1 / p^{m}}} k(V)^{1 / p^{\infty}}=\mathfrak{a}_{n, m} \otimes_{A^{1 / p^{m}}} k\left(V^{\infty}\right)=\psi_{\infty}^{n}\left(\left(L_{k\left(V^{\infty}\right)}^{\frac{p^{n e}-1}{p^{e}-1}}\right)^{1 / p^{n e}}\right), \\
\mathfrak{a}_{m, m} \otimes_{A^{1 / p^{m}}} k(V)^{1 / p^{\infty}}=\mathfrak{a}_{m, m} \otimes_{A^{1 / p^{m}}} k\left(V^{\infty}\right)=\psi_{\infty}^{m}\left(\left(L_{k\left(V^{\infty}\right)}^{\frac{p^{m e}-1}{p^{e}-1}}\right)^{1 / p^{m e}}\right) .
\end{gathered}
$$




\section{$F$-SINGULARITIES IN FAMILIES}

Thus,

$$
\mathfrak{a}_{n, m} \otimes_{A} k(V)^{1 / p^{\infty}}=\mathfrak{a}_{m, m} \otimes_{A} k(V)^{1 / p^{\infty}}
$$

since $m>n \geqslant n_{0}$. It immediately follows that

$$
\mathfrak{a}_{n, m} \otimes_{A^{1 / p^{m}}} k(V)^{1 / p^{m}}=\mathfrak{a}_{m, m} \otimes_{A^{1 / p^{m}}} k(V)^{1 / p^{m}}
$$

since $k(V)^{1 / p^{m}} \subseteq k(V)^{1 / p^{\infty}}$ is a faithfully flat extension. But now by generic freeness [Eis95, Theorem 14.4], for a fixed $m,(3.1)$ follows for some open set $U_{n, m}$ (in the case that $V$ is affine, which we can certainly reduce to, we can invert a single element of $A$ to form $U_{n, m}$ ).

We now vary $m$. Choose a $U=U_{n, n+1}$ that works for $m=n+1$ and consider the diagram

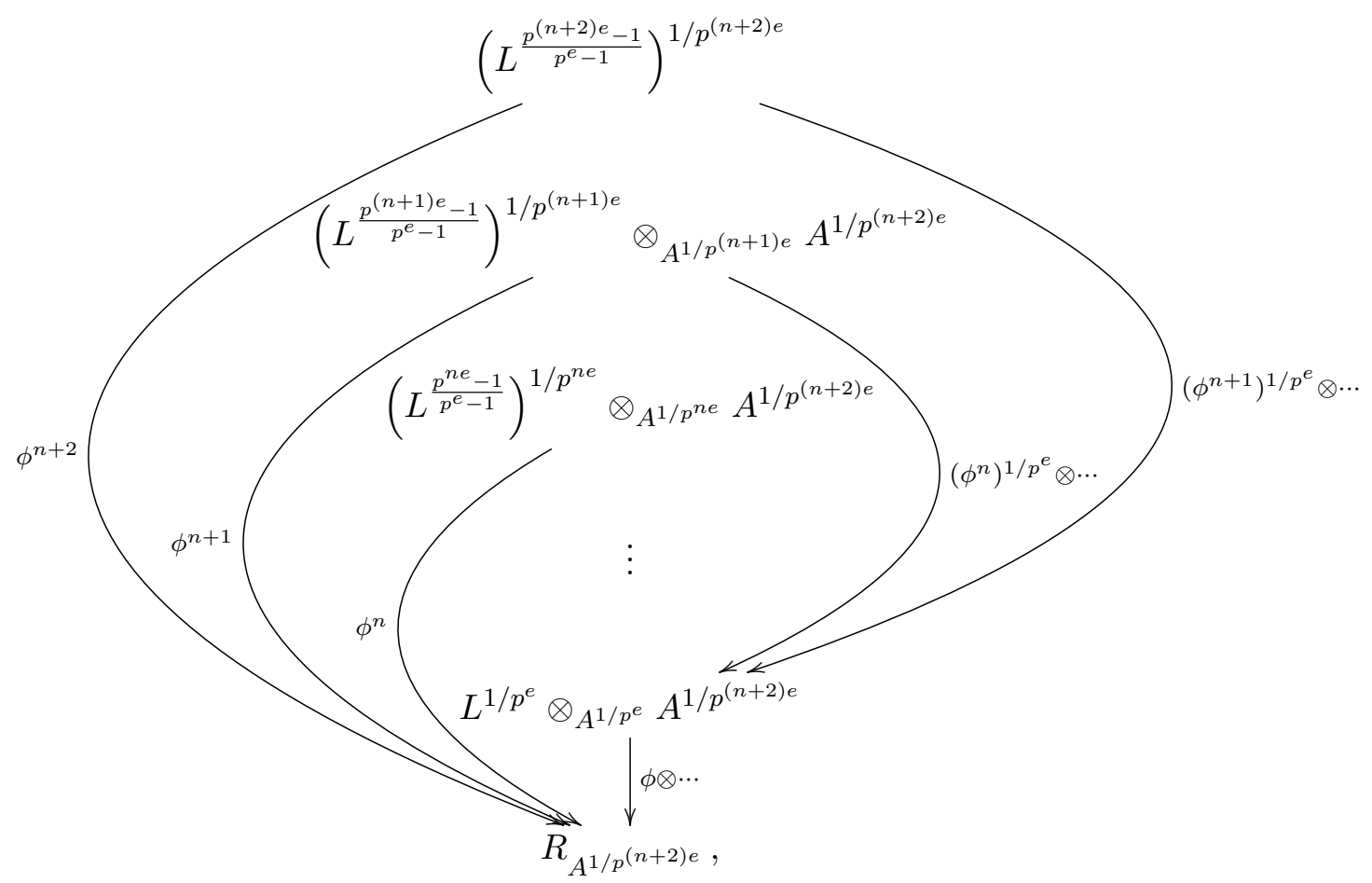

where the maps labeled $\left(\phi^{n}\right)^{1 / p^{e}} \otimes \cdots$ and $\left(\phi^{n+1}\right)^{1 / p^{e}} \otimes \cdots$ are induced as in $(2.3)$. We know that over $U$, the maps $\phi^{n+1}$ and $\phi^{n}$ have the same image. Therefore, so do $\left(\phi^{n}\right)^{1 / p^{e}} \otimes \cdots$ and $\left(\phi^{n+1}\right)^{1 / p^{e}} \otimes \cdots$, again over $U$ (since tensor is right exact). But then, composing with $(\phi \otimes \cdots)$ one more time, we know that $\phi^{n+1}=(\phi \otimes \cdots) \circ\left(\left(\phi^{n}\right)^{1 / p^{e}} \otimes \cdots\right)$ and $\phi^{n+2}=(\phi \otimes \cdots) \circ\left(\left(\phi^{n+1}\right)^{1 / p^{e}} \otimes \cdots\right)$ also have the same image over $U$. Thus, they also share the image with $\phi^{n}$ over $U$. Hence, if $\left.\mathfrak{a}_{n, n+1}\right|_{U}=\left.\mathfrak{a}_{n+1, n+1}\right|_{U}$, then $\left.\mathfrak{a}_{n, n+2}\right|_{U}=\left.\mathfrak{a}_{n+2, n+2}\right|_{U}$. We iterate this to obtain that $\left.\mathfrak{a}_{n, m}\right|_{U}=$ $\left.\mathfrak{a}_{m, m}\right|_{U}$, which is exactly the statement of the proposition.

We give three examples of these $\sigma_{n}$ and $U_{n}$. In the first example, we show that it is possible that the images $\sigma_{n}(X / V, \phi)$ never stabilize in the sense of Proposition 3.3 on all of $V$ but only over an open set. We do the same in the second example, but with respect to a more interesting choice of $X$ and $\phi$. Finally, we give an example where stabilization occurs at $n=2$ (instead of at $n=1)$.

EXAMPLE 3.4. Fix $k$ to be an algebraically closed field of characteristic $p>2$, set $A=k[t]$, and set $R=k[x, t]$ with the obvious map $X \rightarrow V$. Let $\phi: R^{1 / p}=k\left[x^{1 / p}, t^{1 / p}\right] \rightarrow R_{A^{1 / p}}=k\left[x, t^{1 / p}\right]$ be the composition of the local generator $\beta \in \operatorname{Hom}_{R_{A^{1 / p}}}\left(R^{1 / p}, R_{A^{1 / p}}\right)$ with pre-multiplication by 


\section{Z. Patakfalvi, K. Schwede And W. Zhang}

$t^{1 / p}$ (note that since $\phi$ is $k\left[t^{1 / p}\right]$-linear, this is also post-multiplication by $t^{1 / p}$, and it corresponds to the divisor $\left.\Delta_{\phi}=(1 /(p-1))\{t=0\}\right)$. It easily follows that the image of $\phi$ is $\left\langle t^{1 / p}\right\rangle \subseteq k\left[x, t^{1 / p}\right]$. By tensoring with $\otimes_{k\left[t^{1 / p}\right]} k\left[t^{1 / p^{2}}\right]$, we obtain $\mathfrak{a}_{1,2}=\left\langle t^{1 / p}\right\rangle=t^{1 / p} \cdot k\left[x, t^{1 / p^{2}}\right]$. More generally, we see that $\mathfrak{a}_{1, b}=t^{1 / p} \cdot k\left[x, t^{1 / p^{n}}\right]$. Now, we compute $\mathfrak{a}_{2,2}$ as the image of

$$
k\left[x^{1 / p^{2}}, t^{1 / p^{2}}\right] \stackrel{\phi^{1 / p}}{\longrightarrow} k\left[x^{1 / p}, t^{1 / p^{2}}\right] \stackrel{\phi \otimes \cdots}{\longrightarrow} k\left[x, t^{1 / p^{2}}\right] .
$$

The image of $\phi^{1 / p}$ is $t^{1 / p^{2}}$ and since the map $\phi \otimes \cdots$ is $k\left[t^{1 / p^{2}}\right]$-linear, we see that the composition has image $\left\langle t^{1 / p} \cdot t^{1 / p^{2}}\right\rangle=\left\langle t^{(1+p) / p^{2}}\right\rangle=\mathfrak{a}_{2,2}$. In general, we see that

$$
\mathfrak{a}_{n}=\mathfrak{a}_{n, n}=\left\langle t^{\left(1+p+\cdots+p^{n-1}\right) / p^{n}}\right\rangle .
$$

In particular, while we may take $U_{i}=\mathbb{A}^{1} \backslash\{0\}=\operatorname{Spec} A \backslash\langle t\rangle$, we see that $\mathfrak{a}_{n}=\sigma_{n}(X / V, \phi)$ never stabilizes over all of $V$.

EXAmPle 3.5. Let $f$ be the morphism $X:=\operatorname{Spec}\left(k[x, y, t] /\left(y^{2}+x^{3}+t\right)\right) \rightarrow V:=\operatorname{Spec}(k[t])$. Consider the standard trace map $\phi:\left(F_{X^{1} / V^{1}}\right)_{*} \omega_{X^{1} / V^{1}} \rightarrow \mathscr{O}_{X_{V^{1}}}$, for which $\Delta_{\phi}=0$. This map can be identified with the descent of the following map to the quotient

$$
k[x, y, t] \stackrel{\cdot\left(y^{2}+x^{3}+t\right)^{p-1}}{\longrightarrow} k[x, y, t] \stackrel{\operatorname{Tr}}{\longrightarrow} k[x, y, t],
$$

where $\operatorname{Tr}$ is a $k$-linear map such that

$$
\operatorname{Tr}\left(x^{i} y^{j} t^{l}\right)= \begin{cases}x^{\frac{i+1-p}{p}} y^{\frac{j+1-p}{p}} t^{l} & \text { if } p \mid i+1 \text { and } p \mid j+1, \\ 0 & \text { otherwise. }\end{cases}
$$

Then $\phi^{n}$ is given by

$$
k[x, y, t] \stackrel{\cdot\left(y^{2}+x^{3}+t\right)^{p^{n}-1}}{\longrightarrow} k[x, y, t] \stackrel{\operatorname{Tr}_{n}}{\longrightarrow} k[x, y, t],
$$

where $\operatorname{Tr}_{\mathrm{n}}$ is a $k$-linear map such that

$$
\operatorname{Tr}_{n}\left(x^{i} y^{j} t^{l}\right)= \begin{cases}x^{\frac{i+1-p^{n}}{p^{n}}} y^{\frac{j+1-p^{n}}{p^{n}}} t^{l} & \text { if } p^{n} \mid i+1 \text { and } p^{n} \mid j+1, \\ 0 & \text { otherwise. }\end{cases}
$$

Assume that $p$ is a prime such that $p \equiv 1(\bmod 6)$, so that $2,3 \mid p^{n}-1$ for all $n>0$. Let us now try to compute the following number:

$$
d:=\min \left\{c \mid t^{c} \in \operatorname{im} \phi^{n}\right\} .
$$

To have $t^{c} \in \operatorname{im} \phi^{n}$, it is necessary to have a polynomial in the ideal generated by $\left(y^{2}+x^{3}+t\right)^{p^{n}-1}$, of which one of the non-zero monomials is $x^{p^{n}-1} y^{p^{n}-1} t^{c}$. Therefore, $\left(y^{2}+x^{3}+t\right)^{p^{n}-1}$ itself has to have a non-zero monomial which divides $x^{p^{n}-1} y^{p^{n}-1} t^{c}$. That is, there have to be integers $0 \leqslant a \leqslant\left(p^{n}-1\right) / 2$ and $0 \leqslant b \leqslant\left(p^{n}-1\right) / 3$, such that $c=p^{n}-1-a-b$. So, we see that

$$
d \geqslant p^{n}-1-\frac{p^{n}-1}{2}-\frac{p^{n}-1}{3}=\frac{p^{n}-1}{6} .
$$

On the other hand, we claim that $\operatorname{Tr}_{n}\left(y^{2}+x^{3}+t\right)^{p^{n}-1}=t^{\left(p^{n}-1\right) / 6}$, which will show that $d=$ $\left(p^{n}-1\right) / 6$ and also that $\sigma_{n}(X / V, \phi)$ does not stabilize. First, note that $x^{\left(p^{n}-1\right) / 2} y^{\left(p^{n}-1\right) / 3} t^{\left(p^{n}-1\right) / 6}$ is the only monomial in the expansion of $\left(y^{2}+x^{3}+t\right)^{p^{n}-1}$ with non-zero image via $\operatorname{Tr}_{n}$. Indeed, the expansion can contain only monomials of the form $y^{2 a} x^{3 b} t^{p^{n}-1-a-b}$, where $a, b \geqslant 0$ are integers. Hence, higher powers $\left(2 p^{n}-1,3 p^{n}-1\right.$, etc.) of $x$ and $y$ that do not go to zero by $\operatorname{Tr}_{n}$ cannot be 


\section{$F$-SINGULARITIES IN FAMILIES}

obtained. Hence, as we stated, $x^{\left(p^{n}-1\right) / 2} y^{\left(p^{n}-1\right) / 3} t^{\left(p^{n}-1\right) / 6}$ is the only interesting monomial that can show up in the expansion. Last, we have to verify that this monomial has non-zero coefficient in the expansion. That is,

$$
\frac{\left(p^{n}-1\right) !}{\left(\frac{1}{2}\left(p^{n}-1\right)\right) !\left(\frac{1}{3}\left(p^{n}-1\right)\right) !\left(\frac{1}{6}\left(p^{n}-1\right)\right) !} \neq 0 .
$$

Equivalently, we have to show that the power of $p$ in the prime factorization of the numerator is the same as in the denominator. For an arbitrary number $m$, this number for $m$ ! is

$$
\sum_{i=1}^{\infty}\left\lfloor\frac{m}{p^{i}}\right\rfloor \text {. }
$$

Now, assume that $m=\left(p^{n}-1\right) / r$, where $r \mid p-1$. Then the number of times $p$ divides $m$ ! is

$$
\begin{aligned}
\sum_{i=1}^{\infty}\left\lfloor\frac{m}{p^{i}}\right\rfloor & =\sum_{i=1}^{n-1}\left\lfloor\frac{p^{n}-1}{r p^{i}}\right\rfloor=\sum_{i=1}^{n-1}\left\lfloor\sum_{j=0}^{n-1} p^{j} \frac{p-1}{r p^{i}}\right\rfloor=\sum_{i=1}^{n-1}\left\lfloor\sum_{j=0}^{n-1} p^{j-i} \frac{p-1}{r}\right\rfloor \\
& =\sum_{i=1}^{n-1} \sum_{j=i}^{n-1} p^{j-i} \frac{p-1}{r}=\sum_{i=1}^{n-1} \frac{p^{n-i}-1}{p-1} \frac{p-1}{r}=\sum_{i=1}^{n-1} \frac{p^{n-i}-1}{r}=\sum_{i=1}^{n-1} \frac{p^{i}-1}{r} \\
& =\sum_{i=0}^{n-1} \frac{p^{i}-1}{r}=\frac{p^{n}-1}{r} .
\end{aligned}
$$

Therefore, $p$ divides $p^{n}-1$ times both $\left(p^{n}-1\right)$ ! and $\left(\frac{1}{2}\left(p^{n}-1\right)\right) !\left(\frac{1}{3}\left(p^{n}-1\right)\right) !\left(\frac{1}{6}\left(p^{n}-1\right)\right)$ !, as claimed earlier. Summarizing, $t^{\left(p^{n}-1\right) / 6}$ is the smallest power of $t$ that is in $\sigma_{n}(X / V, \phi)$. Hence, there is no stabilization over all of $V$.

Based on this example, one might ask the following.

QUESTION 3.6. Is there a relation between the asymptotics of the $\sigma_{n}$ over the non-stable locus and the singularities of those fibers (for example, the F-pure threshold)?

We now give an example that stabilizes at the second step (over the entire base).

EXAmple 3.7 (cf. [MY09, Example 4.7]). Fix $k$ to be an algebraically closed field of characteristic $p>2$, set $A=k[t]$, and set $R=k[x, t]$ with the obvious map $X \rightarrow V$. Let $\phi: R^{1 / p}=k\left[x^{1 / p}, t^{1 / p}\right] \rightarrow R_{A^{1 / p}}=k\left[x, t^{1 / p}\right]$ be the composition of the local generator $\beta \in$ $\operatorname{Hom}_{R_{A^{1 / p}}}\left(R^{1 / p}, R_{A^{1 / p}}\right)$ with pre-multiplication by $\left(x^{p^{2}}+t\right)^{1 / p}=f^{1 / p}$ (which corresponds to $\Delta_{\phi}=(1 /(p-1))\left\{x^{p^{2}}=t\right\}$ restricting to $\left(p^{2} /(p-1)\right)\left\{x=\lambda^{1 / p^{2}}\right\}$ on the fiber over $\left.t=\lambda\right)$. Note that in $R_{A^{1 / p}}$ or $R^{1 / p}$, the map $f^{1 / p}$ can be written as $x^{p}+t^{1 / p}$. In particular, $f^{1 / p}$ is already an element of $R_{A^{1 / p}}$. Therefore, since $\phi$ is $R_{A^{1 / p}}$-linear and $\beta$ is clearly surjective, the image of $\phi$ is just $\left\langle f^{1 / p}\right\rangle=\sigma_{1}(X / V, \phi)=\left\langle x^{p}+t^{1 / p}\right\rangle$.

On the other hand, we now compose $\phi$ with itself as described above. In this case, $\phi^{2}: R^{1 / p^{2}} \rightarrow$ $R_{A^{1 / p^{2}}}$ is induced by taking the generator $\beta^{2}: R^{1 / p^{2}} \rightarrow R_{A^{1 / p^{2}}}$ and pre-multiplying by $f^{(p+1) / p^{2}}$. Now, $f^{(p+1) / p^{2}}=\left(x^{1}+t^{1 / p^{2}}\right)^{p+1}$. This again is already an element of $R_{A^{1 / p^{2}}}$, and so by the same argument as above, we see that

$$
\sigma_{2}(X / V, \phi)=\left\langle x+t^{1 / p^{2}}\right\rangle^{p+1}=\sigma_{1}(X / V, \phi) \cdot\left\langle x+t^{1 / p^{2}}\right\rangle=\left\langle f^{(1+p) / p^{2}}\right\rangle .
$$




\section{Z. Patakfalvi, K. Schwede And W. Zhang}

Next, we form $\phi^{3}$. In this case, it is obtained by taking a generator $R^{1 / p^{3}} \rightarrow R_{A^{1 / p^{3}}}$ and pre-multiplying by $f^{\left(1+p+p^{2}\right) / p^{3}}$. However, this time $f^{\left(1+p+p^{2}\right) / p^{3}}$ is not contained in $R_{A^{1 / p^{3}}}$, and so we cannot argue as above. However, $f$ still has a $p^{2}$ rd root in $R_{A^{1 / p^{3}}}$, and so

$$
\phi^{3}\left(\left\langle f^{\frac{\left.1+p+p^{2}\right)}{p^{3}}}\right\rangle\right)=\phi^{3}\left(f^{p(1+p)}\left\langle f^{1 / p^{3}}\right\rangle\right)=f^{\frac{1+p}{p^{2}}} \phi^{3}\left(\left\langle f^{1 / p^{3}}\right\rangle\right) .
$$

So we must only compute $\phi^{3}\left(\left\langle f^{1 / p^{3}}\right\rangle\right)$. Now, we may take $\phi^{3}: k\left[x^{1 / p^{3}}, t^{1 / p^{3}}\right] \rightarrow k\left[x, t^{1 / p^{3}}\right]$ as the map which sends $x^{\left(p^{3}-1\right) / p^{3}}$ to 1 and other monomials in $x$ to 0 (those monomials form a basis for $R^{1 / p^{3}}$ over $\left.R_{A^{1 / p^{3}}}\right)$. It is thus obvious that $\phi^{3}\left(\left\langle f^{1 / p^{3}}\right\rangle\right)=k\left[x, t^{1 / p^{3}}\right]=R_{A^{1 / p^{3}}}$. In particular,

$$
\sigma_{3}(X / V, \phi)=\left\langle f^{\frac{1+p}{p^{2}}}\right\rangle=\sigma_{2}(X / V, \phi) \otimes_{A^{1 / p^{2}}} A^{1 / p^{3}} .
$$

We now obtain a surprising base change statement.

Proposition 3.8. Suppose that $T \rightarrow V$ is a map from an excellent integral scheme with a dualizing complex; then, using the notation of Section 2.2,

$$
\operatorname{im}\left(\left(q_{n e}\right)^{*} \sigma_{n}(X / V, \phi) \rightarrow \mathscr{O}_{X_{T} n e}\right)=\sigma_{n}(X / V, \phi) \cdot \mathscr{O}_{X_{T}{ }^{n e}}=\sigma_{n}\left(X_{T} / T, \phi_{T}\right) .
$$

Furthermore, if $U$ satisfies condition (3.1) from Proposition 3.3, then $W=g^{-1}(U) \subseteq T$ satisfies the same condition for $\sigma_{n}\left(X_{T} / T, \phi_{T}\right)$.

Proof. Indeed, images of maps are compatible with arbitrary base change by the right exactness of tensor. Thus, the first statement follows immediately. The second statement follows from the first since if the two images $\mathfrak{a}_{n, n}$ and $\mathfrak{a}_{n-1, n}$ are equal, they are also equal after base change.

Theorem 3.9 (Base change for $\sigma_{n}$ ). There exists an integer $N \geqslant 0$ such that for all points $s \in V$, we have $N \geqslant n_{\sigma\left(\phi_{s}\right), k(s)}$. In other words, we have both that $\sigma_{n}(X / V, \phi) \cdot \mathscr{O}_{X_{s} n e}=\sigma_{n}\left(X_{s} / s, \phi_{s}\right)$ (which always holds) and also that for all $m \geqslant n \geqslant N$,

$$
\sigma_{n}(X / V, \phi) \otimes_{V^{n}} k(s)^{1 / p^{m}}=\sigma_{m}\left(X_{s} / s, \phi_{s}\right) .
$$

Proof. Of course, the statement already holds on $U$ with respect to some integer $N_{0}$. Set $V_{1}^{\prime}:=$ $V \backslash U$ to be the complement with reduced scheme structure, and let

$$
i_{1}: V_{1}=\left(V_{1}^{\prime}\right)_{\mathrm{reg}} \hookrightarrow V_{1}^{\prime}
$$

be the regular locus. We notice that $V_{1}$ has dimension strictly less than $\operatorname{dim} V$. We consider the base change $X_{V_{1}} \rightarrow V_{1}$. Each fiber of $X_{V_{1}} \rightarrow V_{1}$ is isomorphic to a fiber of $X \rightarrow V$. Choose an open set $U_{1} \subseteq V_{1}$ for which the statement holds for some integer $N_{1}$.

Now, fix $V_{2}^{\prime}=V_{1}^{\prime} \backslash U_{1}$ and $i_{2}: V_{2}=\left(V_{2}^{\prime}\right)_{\text {reg }} \hookrightarrow V_{2}^{\prime}$ to be the regular locus and repeat. This process terminates by Noetherian induction.

Setting $N=\max \left\{N_{0}, N_{1}, N_{2}, \ldots\right\}$ completes the proof.

Now, suppose that $s \in V$ is a perfect point; see Definition 2.2. It follows that $X_{s} / s$ is a variety over a perfect field and so since $s^{e} \cong s$, we have $X_{s^{e}} \cong X_{s}$. Thus, we can identify $\phi_{s}: L_{s}^{1 / p^{e}} \rightarrow R_{s^{e}}$ with a $p^{-e}$-linear map $\psi_{s}$ as in Section 2.3. Thus, under these identifications, $\bigcap_{n \geqslant 0} \sigma_{n}\left(X_{s} / s, \phi\right)=$ $\sigma\left(X_{s}, \psi_{s}\right)$. However, if we have the stabilization $\sigma_{n}\left(X_{s} / s, \phi\right) \cong \sigma_{n}\left(X_{s} / s, \phi\right) \otimes_{k(s)^{1 / p^{n e}}} k(s)^{1 / p^{m e}} \cong$ $\sigma_{m}\left(X_{s} / s, \phi\right)$ for every $m \geqslant n$, the equality $\sigma_{n}\left(X_{s} / s, \phi\right)=\sigma\left(X_{s}, \psi\right)$ holds. Combining this with our previous work, we obtain the following corollary. 


\section{$F$-SINGULARITIES IN FAMILIES}

Corollary 3.10. With notation as above, there exists an integer $N \geqslant 0$ such that

$$
\sigma_{n}(X / V, \phi) \cdot \mathscr{O}_{X_{s}^{n e}}=\sigma\left(X_{s}, \psi_{s}\right)
$$

for all perfect points $s \in V$ and $n \geqslant N$.

\subsection{Iterated non- $F$-pure ideals versus the absolute non- $F$-pure ideal}

We now compare the iterated non- $F$-pure ideal with the absolute non- $F$-pure ideal. First however, we make the following assumption.

Convention 3.11. In this subsection, Section 3.1, we always assume that our base $V$ is an $F$-pure, quasi-Gorenstein (that is, $\omega_{V}$ is a line bundle), $F$-finite, integral scheme.

Let $\Psi_{V}^{e}: F_{*}^{e} \mathscr{O}_{V}\left(\left(1-p^{e}\right) K_{V}\right) \rightarrow \mathscr{O}_{V}$ be a map generating $\mathscr{H} \mathrm{om}_{V}\left(F_{*}^{e} \mathscr{O}_{V}\left(\left(1-p^{e}\right) K_{V}\right), \mathscr{O}_{V}\right)$ as an $F_{*}^{e} \mathscr{O}_{V}$-module. By taking $p^{n e}$ th roots, applying $f^{-1}$, and then writing the $A$-module $M:=$ $f^{-1}\left(\mathscr{O}_{V}\left(\left(1-p^{e}\right) K_{V}\right)\right)$, we obtain

$$
\left(\Phi_{A}^{e}\right)^{1 / p^{n e}}: M^{1 / p^{(n+1) e}}=f^{-1} F_{*}^{(n+1) e}\left(\mathscr{O}_{V}\left(\left(1-p^{e}\right) K_{V}\right)\right) \rightarrow f^{-1} F_{*}^{n e} \mathscr{O}_{V}=A^{1 / p^{n e}}
$$

We tensor with $L^{1 / p^{e}}$ over $A^{1 / p^{e}}$ and so obtain

$$
\Phi^{\prime}: L^{1 / p^{e}} \otimes_{A^{1 / p^{e}}} M^{1 / p^{(n+1) e}} \rightarrow L^{1 / p^{e}} \otimes_{A^{1 / p^{e}}} A^{1 / p^{n e}} .
$$

Set $N=L \otimes_{A} M^{1 / p^{n e}}$. We observe that $N$ is a line bundle on $X_{V^{n e}}$. In fact, if $q_{1}: X_{V^{n e}}=$ $X \times_{V} V^{n e} \rightarrow X$ is the first projection and $q_{2}: X_{V^{n e}} \rightarrow V^{n e}$ is the second projection, then

$$
N=q_{1}^{*} L \otimes_{\mathscr{O}_{X_{V}} \text { e }} q_{2}^{*} \mathscr{O}_{V^{n e}}\left(\left(1-p^{e}\right) K_{V^{n e}}\right)
$$

and so it follows for any integer $l>0$ that

$$
N^{l} \cong q_{1}^{*} L^{l} \otimes_{\mathscr{O}_{X_{V} e}} q_{2}^{*} \mathscr{O}_{V^{n e}}\left(l\left(1-p^{e}\right) K_{V^{n e}}\right)=L^{l} \otimes_{A}\left(M^{l}\right)^{1 / p^{n e}} .
$$

Finally, we compose (3.3) with $\phi^{\prime}:=\phi \otimes_{A^{1 / p^{e}}} A^{1 / p^{n e}}$ to construct

$$
\begin{aligned}
\gamma: N^{1 / p^{e}} & =L^{1 / p^{e}} \otimes_{A^{1 / p^{e}}} M^{1 / p^{(n+1) e}} \stackrel{\Phi^{\prime}}{\rightarrow} L^{1 / p^{e}} \otimes_{A^{1 / p^{e}}} A^{1 / p^{n e}} \\
& \stackrel{\phi^{\prime}}{\rightarrow} R_{A^{1 / p^{e}}} \otimes_{A^{1 / p^{e}}} A^{1 / p^{n e}}=R \otimes_{A} A^{1 / p^{n e}}=R_{A^{1 / p^{n e}}} .
\end{aligned}
$$

This is a map from an invertible sheaf on $X_{V^{(n+1) e}}^{e}=\left(X_{V^{n e}}\right)^{e}$ to the structure sheaf on $X_{V^{n e}}$. In particular, it is a map such as one studied in [BS13, Section 4].

Let us point out an alternate way to construct $\gamma$. Indeed, take

$$
\begin{aligned}
\phi^{\prime \prime}:=\phi \otimes_{A^{1 / p^{e}}} M^{1 / p^{(n+1) e}}: L^{1 / p^{e}} \otimes_{A^{1 / p^{e}}} M^{1 / p^{(n+1) e}} & \rightarrow R_{A^{1 / p^{e}}} \otimes_{A^{1 / p^{e}}} M^{1 / p^{(n+1) e}} \\
& \cong R \otimes_{A} M^{1 / p^{(n+1) e}} .
\end{aligned}
$$

We can then compose this with $R_{A^{1 / p^{e}}} \otimes_{A^{1 / p^{e}}}\left(\Phi_{A}^{e}\right)^{1 / p^{n e}}=\left(\Phi_{A}^{e}\right)^{\prime}$ to obtain

$$
\begin{aligned}
N^{1 / p^{e}}=L^{1 / p^{e}} \otimes_{A^{1 / p^{e}}} M^{1 / p^{(n+1) e}} & \stackrel{\phi^{\prime \prime}}{\longrightarrow} R_{A^{1 / p^{e}}} \otimes_{A^{1 / p^{e}}} M^{1 / p^{(n+1) e}} \\
& \stackrel{\left(\Phi_{A}^{e}\right)^{\prime}}{\longrightarrow} R_{A^{1 / p^{e}}} \otimes_{A^{1 / p^{e}}} A^{1 / p^{n e}}=R_{A^{1 / p^{n e}}} .
\end{aligned}
$$

This composition is easily seen to coincide with $\gamma$.

We can then compose $\gamma$ with itself $m$ times as in [BS13, Section 4] or [Sch09]. We recall this construction for the benefit of the reader. To construct $\gamma^{2}$, we tensor the map

$$
\gamma: N^{1 / p^{e}} \rightarrow R \otimes_{A} A^{1 / p^{n e}}=R_{A^{1 / p^{n e}}}
$$




\section{Z. Patakfalvi, K. Schwede And W. Zhang}

with the line bundle $N$ over $R_{A^{1 / p^{n e}}}$, take $p^{e}$ th roots, and then compose it with $\gamma$ to obtain

$$
\gamma^{2}:\left(N^{1+p^{e}}\right)^{1 / p^{2 e}} \rightarrow N^{1 / p^{e}} \stackrel{\gamma}{\rightarrow} R \otimes_{A} A^{1 / p^{n e}} .
$$

Recursively, we can construct

$$
\begin{aligned}
\gamma^{m}:\left(N^{\frac{p^{m e}-1}{p^{e}-1}}\right)^{1 / p^{m e}} & =\left(L^{\frac{p^{m e}-1}{p^{e}-1}}\right)^{1 / p^{m e}} \otimes_{A^{1 / p^{m e}}}\left(M^{\frac{p^{m e}-1}{p^{e}-1}}\right)^{1 / p^{(n+m) e}} \\
& \rightarrow R_{A^{1 / p^{n e}}}
\end{aligned}
$$

We are now in a position to relate the absolute $\sigma\left(X_{V^{1 / p^{n e}}}, \gamma\right)$ with the relative $\sigma_{n}(X / V, \phi)$.

First, we observe that for each $m \leqslant n$, the map $\Phi_{A}^{m}$ induces maps

$$
\begin{aligned}
\mu_{m}:\left(N^{\frac{p^{m e}-1}{p^{e}-1}}\right)^{1 / p^{m e}} & =\left(L^{\frac{p^{m e}-1}{p^{e}-1}}\right)^{1 / p^{m e}} \otimes_{A^{1 / p^{m e}}}\left(M^{\frac{p^{m e}-1}{p^{e}-1}}\right)^{1 / p^{(n+m) e}} \\
& \stackrel{\cdots \otimes\left(\Phi_{A}^{m}\right)^{1 / p^{n e}}}{\longrightarrow}\left(L^{\frac{p^{m e}-1}{p^{e}-1}}\right)^{1 / p^{m e}} \otimes_{A^{1 / p^{m e}}} A^{1 / p^{n e}} .
\end{aligned}
$$

Furthermore, these maps are surjective since $\Phi_{A}^{m}$ is.

LEMma 3.12. Assuming Convention 3.11, we have the factorization of $\gamma^{n}$ and $\gamma^{n-1}$ indicated by the diagram below:

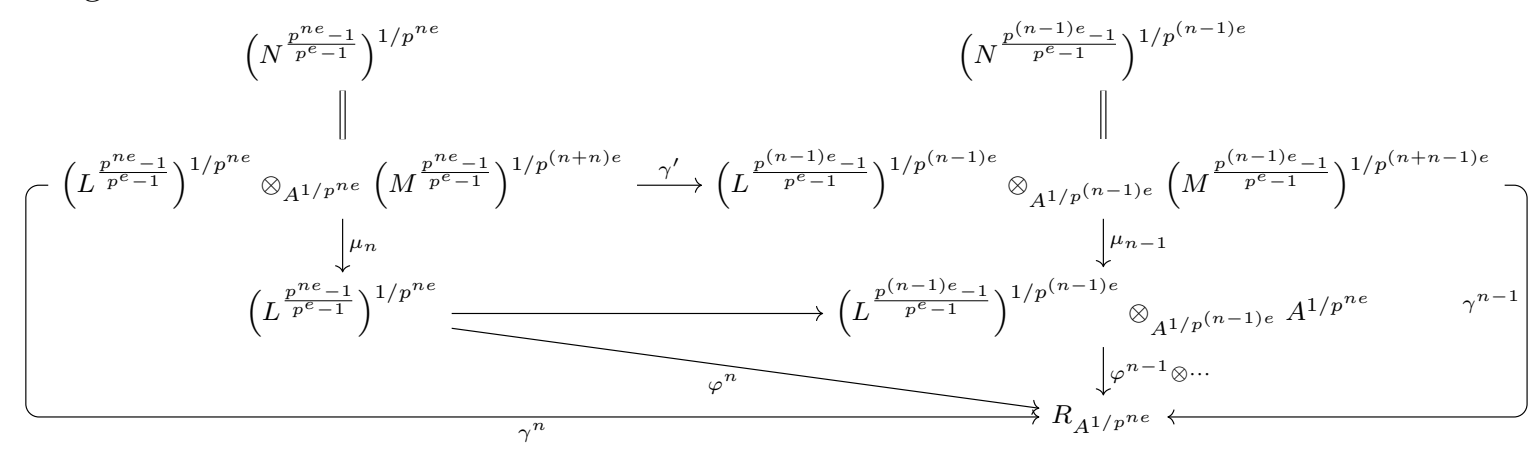

where the arrow $\gamma^{\prime}$ is induced by $\gamma$ and $\phi^{n-1} \otimes \cdots$ is simply $\phi^{n-1} \otimes_{A^{1 / p}(n-1) e} A^{1 / p^{n e}}$. Furthermore, since $\mu_{n-1}$ is surjective, we have

$$
\operatorname{im}\left(\gamma^{n-1}\right)=\mathfrak{a}_{n-1, n}=\operatorname{im}\left(\phi^{n-1} \otimes_{A^{1 / p}(n-1) e} A^{1 / p^{n e}}\right) .
$$

Likewise, since $\mu_{n}$ is surjective, we have

$$
\operatorname{im}\left(\gamma^{n}\right)=\mathfrak{a}_{n, n}=\mathfrak{a}_{n}=\operatorname{im}\left(\phi^{n}\right) .
$$

Proof. The two equalities are immediate from the surjectivity of $\mu_{n-1}$ and $\mu_{n}$ and the commutativity of our diagram. The surjectivity of $\mu_{n-1}$ and $\mu_{n}$ is clear since $M^{1 / p^{(n+1) e}} \rightarrow A^{1 / p^{n e}}$ is surjective and tensor is right exact. It remains to prove the commutativity of our diagram. It suffices to prove the commutativity of the square

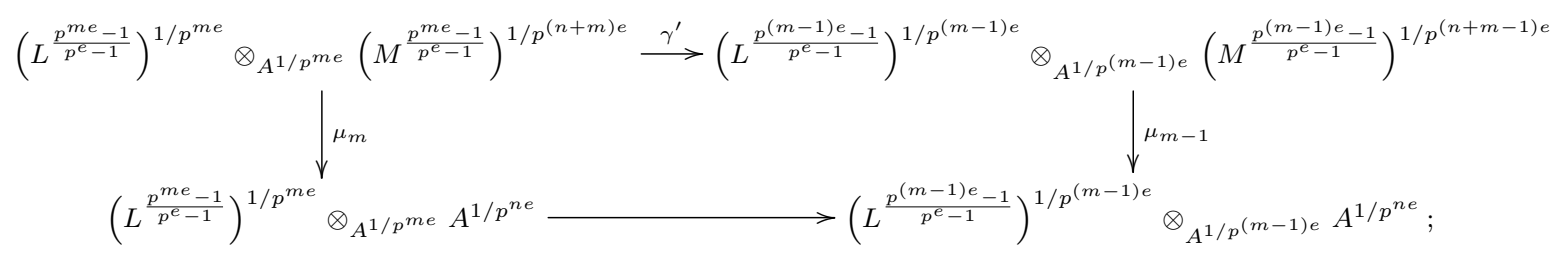

we will prove it by induction on $m$. It is clear that if the above square is commutative for $m$, then, by tensoring it with $L \otimes_{R}(-) \otimes_{A^{1 / p^{n e}}} M^{1 / p^{(n+1) e}}$ and taking $p^{e}$ th roots, we will have the 


\section{$F$-SINGULARITIES IN FAMILIES}

commutativity for $m+1$. Hence, it remains to prove the commutativity when $m=2$. To this end, we will denote the map $M^{1 / p^{(n+1) e}} \rightarrow A^{1 / p^{n e}}$ by $\alpha$. Then $\alpha$ induces a map

$$
\begin{aligned}
\alpha^{\prime}=(\mathrm{id} \otimes \alpha)^{1 / p^{e}}:\left(M^{\frac{p^{2 e}-1}{p^{e}-1}}\right)^{1 / p^{(n+2) e}} & =\left(M^{1 / p^{n e}} \otimes_{A^{1 / p^{n e}}} M^{1 / p^{(n+1) e}}\right)^{1 / p^{e}} \\
& \rightarrow M^{1 / p^{n e}} \otimes_{A^{1 / p^{n e}}} A^{1 / p^{n e}}=M^{1 / p^{(n+1) e}} .
\end{aligned}
$$

It is clear that $\alpha \circ \alpha^{\prime}=\alpha^{2}$. Likewise, the map $\varphi: L^{1 / p^{e}} \rightarrow R \otimes_{A} A^{1 / p^{e}}$ induces

$$
\varphi^{\prime}=(\operatorname{id} \otimes \varphi)^{1 / p^{e}}:\left(L^{\frac{p^{2 e}-1}{p^{e}-1}}\right)^{1 / p^{2 e}}=\left(L^{1 / p^{e}} \otimes_{R^{1 / p^{e}}} L^{1 / p^{2 e}}\right)^{1 / p^{e}} \rightarrow L^{1 / p^{e}} \otimes_{A^{1 / p^{e}}} A^{1 / p^{2 e}} .
$$

We will analyze the four maps involved in the square. The left vertical map

$$
\mu_{2}:\left(L^{\frac{p^{2 e}-1}{p^{e}-1}}\right)^{1 / p^{2 e}} \otimes_{A^{1 / p^{2 e}}}\left(M^{\frac{p^{2 e}-1}{p^{e}-1}}\right)^{1 / p^{(n+2) e}} \rightarrow\left(L^{\frac{p^{2 e}-1}{p^{e}-1}}\right)^{1 / p^{2 e}}
$$

is given by id $\otimes\left(\alpha \circ \alpha^{\prime}\right)$; similarly, the right vertical map $\mu_{1}$ is id $\otimes \alpha$. The top horizontal map is given by

$$
\begin{aligned}
\left(L^{\frac{p^{2 e}-1}{p^{e}-1}}\right)^{1 / p^{2 e}} \otimes_{A^{1 / p^{2 e}}}\left(M^{\frac{p^{2 e}-1}{p^{e}-1}}\right)^{1 / p^{(n+2) e}} & \stackrel{\varphi^{\prime} \otimes \alpha^{\prime}}{\longrightarrow} L^{1 / p^{e}} \otimes_{A^{1 / p^{e}}} A^{1 / p^{2 e}} \otimes_{A^{1 / p^{2 e}}} M^{1 / p^{(n+1) e}} \\
& \stackrel{\sim}{\rightarrow} L^{1 / p^{e}} \otimes_{A^{1 / p^{e}}} M^{1 / p^{(n+1) e}} .
\end{aligned}
$$

The bottom horizontal map is given by

$$
\begin{aligned}
\left(L^{\frac{p^{2 e}-1}{p^{e}-1}}\right)^{1 / p^{2 e}} \otimes_{A^{1 / p^{2 e}}} A^{1 / p^{n e}} & \stackrel{\varphi^{\prime} \otimes \mathrm{id}}{\longrightarrow} L^{1 / p^{e}} \otimes_{A^{1 / p^{e}}} A^{1 / p^{2 e}} \otimes_{A^{1 / p^{2 e}}} A^{1 / p^{n e}} \\
& \stackrel{\sim}{\rightarrow} L^{1 / p^{e}} \otimes_{A^{1 / p^{e}}} A^{1 / p^{n e}} .
\end{aligned}
$$

Now, given an arbitrary $x \otimes z \in\left(L^{\frac{p^{2 e}-1}{p^{e}-1}}\right)^{1 / p^{2 e}} \otimes_{A^{1 / p^{2 e}}}\left(M^{\frac{p^{2 e}-1}{p^{e}-1}}\right)^{1 / p^{(n+2) e}}$, write $\varphi^{\prime}(x)=$ $\sum_{i} x_{i} \otimes y_{i}$ with $x_{i} \in L^{1 / p^{e}}$ and $y_{i} \in A^{1 / p^{2 e}}$. Then, on one hand, if we follow the top horizontal map and then the right vertical map, we have

$$
x \otimes z \mapsto\left(\sum_{i} x_{i} \otimes y_{i}\right) \otimes \alpha^{\prime}(z) \mapsto \sum_{i} x_{i} \otimes y_{i} \alpha^{\prime}(z) \mapsto \sum_{i} x_{i} \alpha\left(y_{i} \alpha^{\prime}(z)\right)=\sum_{i} x_{i} \otimes y_{i} \alpha\left(\alpha^{\prime}(z)\right),
$$

where the last equality holds since $\alpha$ is $A^{1 / p^{n e}}$-linear and hence $A^{1 / p^{2 e}}$-linear.

On the other hand, if we follow the other path (that is, the left vertical map first and then the bottom horizontal map), we have

$$
x \otimes z \mapsto x \otimes \alpha^{\prime}(z) \mapsto x \otimes \alpha\left(\alpha^{\prime}(z)\right) \mapsto\left(\sum_{i} x_{i} \otimes y_{i}\right) \otimes \alpha\left(\alpha^{\prime}(z)\right) \mapsto \sum_{i} x_{i} \otimes y_{i} \alpha\left(\alpha^{\prime}(z)\right)
$$

This proves that the square is indeed commutative when $m=2$ and concludes the proof of our lemma.

Theorem 3.13. With notation as in Convention 3.11 and below it, choose $n>n_{0}=n_{\sigma(\phi), k(V)}$. Then, there exists a dense open set $U \subseteq V \cong V^{e}$ with $W=f^{-1}(U) \subseteq X$ such that

$$
\left.\sigma\left(X_{V^{1 / p^{n e}}}, \gamma\right)\right|_{W}=\left.\sigma_{n}(X / V, \phi)\right|_{W} .
$$

Furthermore, shrinking $U$ further if necessary, we can require for all perfect points $u \in U$ that

$$
\sigma\left(X_{V^{1 / p^{n e}}}, \gamma\right) \cdot \mathscr{O}_{X_{u}}=\sigma_{n}\left(X_{u} / u, \phi_{u}\right)=\sigma\left(X_{u}, \psi_{u}\right),
$$

where $\psi_{u}$ is $\phi_{u}$ viewed as an absolute $p^{-e}$-linear map. 


\section{Z. Patakfalvi, K. Schwede And W. Zhang}

Proof. The second statement is immediate from the first by Proposition 3.8, so we need only prove the first statement. By the diagram in Lemma 3.12 and applying Proposition 3.3, we see that

$$
\left.\operatorname{im}\left(\gamma^{n}\right)\right|_{W}=\left.\mathfrak{a}_{n, n}\right|_{W}=\left.\mathfrak{a}_{n-1, n}\right|_{W}=\left.\operatorname{im}\left(\gamma^{n-1}\right)\right|_{W}
$$

for all $n>n_{0}$. Hence, $\left.\operatorname{im}\left(\gamma^{n}\right)\right|_{W}=\left.\sigma\left(X_{V^{1 / p^{n e}}}, \gamma\right)\right|_{W}$, and the result follows.

Convention 3.14. Assuming everything in Convention 3.11, we additionally assume that $\phi$ is relatively divisorial.

Before we proceed, we prove a lemma about interpreting maps as divisors in the relative versus absolute setting.

Lemma 3.15. With notation as in Convention 3.14, let $\Delta_{\phi}$ be the divisor corresponding to $\phi$ as in Definition 2.8. Then $\Delta_{\gamma}$, the divisor on $X_{V^{n e}}$ corresponding to $\gamma$ as in [BS13, Section 4], is equal to $h^{*} \Delta$, where $h: X_{V^{n e}}=X \times_{V} V^{n e} \rightarrow X$ is the projection.

Proof. Working off a set of relative codimension 2, and then working locally on $X$ and $V$, we can assume that $L$ and $M$ are trivial, and further assume that $\phi\left(\_\right)=\Phi\left(z^{1 / p^{e}} \cdot{ }_{-}\right)$, where $\Phi$ generates $\mathscr{H} \mathrm{om}_{R_{A^{1 / p^{e}}}}\left(R^{1 / p^{e}}, R_{A^{1 / p^{e}}}\right)$ as an $R^{1 / p^{e}}$-module. Then $\Delta_{\phi}$ is the divisor corresponding to $\left(1 /\left(p^{e}-1\right)\right) \operatorname{div}_{X}(z)$. On the other hand, since the map $\Gamma$ corresponding to $\Phi$ is a composition of generating maps, $\Gamma$ generates $\mathscr{H} \mathrm{om}_{R_{A^{1 / p^{n e}}}}\left(R_{A^{1 / p^{(n+1) e}}}^{1 / p^{e}}, R_{A^{1 / p^{n e}}}\right)$; see for example [Kun86, Appendix F], and so corresponds to the zero divisor. Furthermore, we see that $\gamma\left(\_\right)=\Gamma\left(\bar{z}^{1 / p^{e}}\right.$._ $)$ where $\bar{z}$ is the element corresponding to $z \otimes 1 \in R_{A^{1 / p^{n e}}}$. Hence, $\Delta_{\gamma}=\left(1 /\left(p^{e}-1\right)\right) \operatorname{div}_{X_{V^{n e}}}(\bar{z})$ and the claim follows.

We now need a lemma which says roughly that in an $F$-pure, $F$-finite ring $A$ with maximal ideal $\mathfrak{m}$, at least locally, one can always choose an $\alpha: A^{1 / p^{e}} \rightarrow A$ such that the closed point $V(\mathfrak{m})$ is an $F$-pure center of $\Delta_{\alpha}$. To this end, we introduce the following sets:

$$
\begin{aligned}
M_{e, A} & :=\left\{\phi \in \operatorname{Hom}_{A}\left(A^{1 / p^{e}}, A\right) \mid \phi\left(\mathfrak{m}^{1 / p^{e}}\right) \subseteq \mathfrak{m}\right\} \\
N_{e, A} & :=\left\{\phi \in \operatorname{Hom}_{A}\left(A^{1 / p^{e}}, A\right) \mid \phi\left(A^{1 / p^{e}}\right) \subseteq \mathfrak{m}\right\} .
\end{aligned}
$$

It is straightforward to check that both $M_{e, A}$ and $N_{e, A}$ are $A^{1 / p^{e}}$-submodules of $\operatorname{Hom}_{A}\left(A^{1 / p^{e}}, A\right)$ and that $N_{e, A} \subseteq M_{e, A}$. We now observe the following result.

Lemma 3.16. For any $F$-finite, reduced, $G 1$ and $S 2 \operatorname{ring} A$ with maximal ideal $\mathfrak{m}$, the formation of $M_{e, A}$ and $N_{e, A}$ commutes with localization and completion; that is, if $W$ is a multiplicative system and ^ denotes completion along $\mathfrak{m}$, then

$$
\begin{gathered}
W^{-1} M_{e, A} \cong\left(W^{-1} M\right)_{e, W^{-1} A} \quad \text { and } \quad W^{-1} N_{e, A} \cong\left(W^{-1} N\right)_{e, W^{-1} A} \text { and } \\
M_{e, A} \otimes_{A} \hat{A} \cong M_{e, \hat{A}} \quad \text { and } \quad N_{e, A} \otimes_{A} \hat{A} \cong N_{e, \hat{A}} .
\end{gathered}
$$

The following proof was suggested to us by Manuel Blickle and Kevin Tucker. We believe that there are more general proofs that work without the G1 and S2 hypothesis (but this proof is short).

Proof. We have a natural injective map $\operatorname{Hom}_{A}\left(\mathfrak{m}^{1 / p^{e}}, \mathfrak{m}\right) \hookrightarrow \operatorname{Hom}_{A}\left(\mathfrak{m}^{1 / p^{e}}, A\right)$, but $\operatorname{Hom}_{A}\left(\mathfrak{m}^{1 / p^{e}}, A\right)$ $\cong \operatorname{Hom}_{A}\left(A^{1 / p^{e}}, A\right)$ since both modules are $\mathrm{S} 2$ (since a reflexive module is $\mathrm{S} 2$ in a $\mathrm{G} 1$ and $\mathrm{S} 2$ ring [Har94]). Thus, we have $\operatorname{Hom}_{A}\left(\mathfrak{m}^{1 / p^{e}}, \mathfrak{m}\right) \hookrightarrow \operatorname{Hom}_{A}\left(A^{1 / p^{e}}, A\right)$, the image of which is $M_{e, A}$. 


\section{$F$-SINGULARITIES IN FAMILIES}

Therefore, $M_{e, A}$ clearly commutes with localization and completion since the formation of both Hom sets commutes with localization and completion. Similarly, the formation of $N_{e, A}$ commutes with localization and completion.

Lemma 3.17. Suppose that $A$ is an $F$-pure, $F$-finite, $G 1$ and $S 2$ ring with maximal ideal $\mathfrak{m}$. Then there exists an $A$-linear map $\alpha_{A}: A^{1 / p^{e}} \rightarrow A$ such that $\alpha_{A}$ is surjective and $\alpha_{A}\left(\mathfrak{m}^{1 / p^{e}}\right) \subseteq \mathfrak{m}$.

Proof. We first assume that $A$ is a local ring. In this case, it suffices to show that $N_{e, A} \neq M_{e, A}$. By Lemma 3.16, we may assume that $A$ is complete since $\hat{A}$ is faithfully flat over $A$. By the Cohen structure theorem, there is a ring of formal power series $S=k \llbracket x_{1}, \ldots, x_{n} \rrbracket$ over a coefficient field $k$ of $A$ with surjection $S \rightarrow A$. Write $A=S / I$. Recall that $\operatorname{Hom}_{S}\left(S^{1 / p^{e}}, S\right.$ ) is generated (as an $S^{1 / p^{e}}$-module) by $g: S^{1 / p^{e}} \rightarrow S$, which sends $\left(x_{1} \cdots x_{n}\right)^{\left(p^{e}-1\right) / p^{e}}$ to 1 and other basis monomials (including 1) to zero. By Fedder's lemma [Fed83, Lemma 1.6], each element of $\operatorname{Hom}_{A}\left(A^{1 / p^{e}}, A\right)$ has the form $f\left(u^{1 / p^{e}}-\right)$ with $u \in\left(I^{\left[p^{e}\right]}: I\right)$. Since $A$ is $F$-pure, $\left(I^{\left[p^{e}\right]}: I\right) \not \subset \mathfrak{n}^{\left[p^{e}\right]}$, where $\mathfrak{n}=\left(x_{1}, \ldots, x_{n}\right)$. Let $u$ be an element of $\left(I^{\left[p^{e}\right]}: I\right) \backslash \mathfrak{n}^{\left[p^{e}\right]}$. Then $u$ must have a monomial term $c x_{1}^{a_{1}} \cdots x_{n}^{a_{n}}$ with $c \in k$ and $a_{i}<p^{e}$ for each $i$. Choose such a monomial appearing in $u$ with the least degree and still denote it by $c x_{1}^{a_{1}} \cdots x_{n}^{a_{n}}$. Then it is clear that $\left(x_{1}^{p^{e}-1-a_{1}} \cdots x_{n}^{p^{e}-1-a_{n}}\right) u-$ $c\left(x_{1} \cdots x_{n}\right)^{p^{e}-1} \in \mathfrak{n}^{\left[p^{e}\right]}$. Now, define $\alpha: A^{1 / p^{e}} \rightarrow A$ by $\left.\alpha\left(\_\right)=g\left(\left(x_{1}^{p^{e}-1-a_{1}} \cdots x_{n}^{p^{e}-1-a_{n}} u\right)^{1 / p^{e}}\right)\right)$. Then $\alpha$ is in $M_{e, A} \backslash N_{e, A}$. This completes the local case by Lemma 3.16.

For the non-local case, we have the map $M_{e, A} \rightarrow A$ which is evaluation at 1 . Since the formation of $M_{e, A}$ commutes with localization, this map is surjective if and only if it is surjective locally. The above work proves the result after localizing at $\mathfrak{m}$. However, clearly $\left(M_{e, A}\right)_{\mathfrak{n}}=$ $\left(\operatorname{Hom}_{A}\left(A^{1 / p^{e}}, A\right)\right)_{\mathfrak{n}}$ for prime ideals not equal to $\mathfrak{m}$. The result follows.

For the moment, we work sufficiently locally so that $X=\operatorname{Spec} R$ and $V=\operatorname{Spec} A$ are affine and that $L$ and $M$ are isomorphic to $R$ and $A$, respectively. Continue to assume that $A$ is $F$ pure and quasi-Gorenstein, and fix a point $s \in \operatorname{Spec} A$. Shrinking $V$ if necessary, choose a map $\alpha_{s} \in \operatorname{Hom}_{A}\left(A^{1 / p^{e}}, A\right)$ which is surjective and which satisfies $\alpha\left(\mathfrak{m}_{s}^{1 / p^{e}}\right) \subseteq \mathfrak{m}_{s}$ (whose existence is guaranteed by Lemma 3.17). We write $\alpha_{s}\left(\_\right)=\Phi_{A}^{e}\left(z^{1 / p^{e}}\right.$._ $)$ for some $z \in A$. Note we have an induced map $\overline{\alpha_{s}}:\left(A / \mathfrak{m}_{s}\right)^{1 / p^{e}} \rightarrow A / \mathfrak{m}_{s}$.

We thus induce the following map: $\beta:\left(R_{A^{1 / p^{n e}}}\right)^{1 / p^{e}} \rightarrow R_{A^{1 / p^{n e}}}$, defined by the rule $\beta\left(\_\right)=$ $\gamma\left(z^{1 / p^{(n+1) e}} \cdot \ldots\right)$. Certainly, using the notation of $(3.5)$,

$$
\begin{aligned}
\beta\left(\mathfrak{m}_{s}^{1 / p^{(n+1) e}} \cdot R_{A^{1 / p^{(n+1) e}}}^{1 / p^{e}}\right) & \gamma\left(z^{1 / p^{(n+1) e}} \mathfrak{m}_{s}^{1 / p^{(n+1) e}} \cdot R_{A^{1 / p^{(n+1) e}}}^{1 / p^{e}}\right) \\
& =\left(\phi^{\prime} \circ\left(\left(\Phi_{A}^{e}\right)^{1 / p^{n e}} \otimes_{A^{1 / p^{n e}}} R^{1 / p^{e}}\right)\right)\left(z^{1 / p^{(n+1) e}} \mathfrak{m}_{s}^{1 / p^{(n+1) e}} \cdot R_{A^{1 / p^{(n+1) e}}}^{1 / p^{e}}\right) \\
& =\left(\phi^{\prime} \circ\left(\left(\alpha_{s}\right)^{1 / p^{n e}} \otimes_{A^{1 / p^{n e}}} R^{1 / p^{e}}\right)\right)\left(\mathfrak{m}_{s}^{1 / p^{(n+1) e}} \cdot R_{A^{1 / p^{(n+1) e}}}^{1 / p^{e}}\right) \\
& \subseteq \phi^{\prime}\left(\mathfrak{m}_{s}^{1 / p^{n e}} \cdot R_{A^{1 / p^{n e}}}^{1 / p^{e}}\right) \\
& \subseteq \mathfrak{m}_{s}^{1 / p^{n e}} R_{A^{1 / p^{n e}}}
\end{aligned}
$$

And, hence, we induce a map

$$
\bar{\beta}: R_{\left(A / \mathfrak{m}_{s}\right)^{1 / p}(n+1) e}^{1 / p^{e}} \rightarrow R_{\left(A / \mathfrak{m}_{s}\right)^{1 / p^{n e}}} .
$$

LEMma 3.18. With notation as above, $\Delta_{\bar{\beta}}=\left.\Delta_{\gamma}\right|_{X_{s} \text { e }}$, where the divisors are induced as in [BS13, Section 4]. 


\section{Z. Patakfalvi, K. Schwede And W. Zhang}

Proof. Since $\phi$ is relatively divisorial, by working in relative codimension 1 , it suffices to show the result in the case that $\Delta_{\gamma}$ (and so $\Delta_{\phi}$ ) is trivial (since locally, $\phi$ or $\psi$ is a generator pre-multiplied by the element which determines $\Delta_{\phi}$ or $\Delta_{\psi}$ ). But now consider the composition constructing $\beta$,

$$
R_{A^{1 / p^{(n+1) e}}}^{1 / p^{e}} \stackrel{\cdot z^{1 / p^{(n+1) e}}}{\longrightarrow} R_{A^{1 / p^{(n+1) e}}}^{1 / p^{e}} \stackrel{\Phi^{\prime}}{\rightarrow} R_{A^{1 / p^{n e}}}^{1 / p^{e}} \stackrel{\phi^{\prime}}{\rightarrow} R_{A^{1 / p^{n e}}} .
$$

The composition of the first two maps sends $\mathfrak{m}^{1 / p^{(n+1) e}} \cdot R_{A^{1 / p^{(n+1) e}}}^{1 / p^{e}}$ to $\mathfrak{m}^{1 / p^{n e}} \cdot R_{A^{1 / p^{n e}}}^{1 / p^{e}}$, and modding out by these ideals induces a generating map for

$$
\operatorname{Hom}_{R_{(A / \mathfrak{m})^{1 / p^{n e}}}}\left(R_{(A / \mathfrak{m})^{1 / p^{(n+1) e}}}^{1 / p^{e}}, R_{(A / \mathfrak{m})^{1 / p^{n e}}}^{1 / p^{e}}\right)
$$

via the fact that $\alpha_{s}$ is surjective and so the induced map $(A / \mathfrak{m})^{1 / p^{(n+1) e}} \rightarrow(A / \mathfrak{m})^{1 / p^{n e}}$ is a generating map as well. On the other hand, if $\phi$ is a generating map, so is $\phi^{\prime} \otimes_{A^{1 / p^{n e}}}(A / \mathfrak{m})^{1 / p^{n e}}$ by Lemma 2.17. Since the composition of two generating maps is a generating map, we are done.

We now explain how the absolute $\sigma$ behaves when restricting to fibers. First, we need two lemmas.

Lemma 3.19. With notation as above and $v \in V$ a closed point,

$$
\sigma\left(X_{V^{n e}}, \Delta_{\gamma}\right) \cdot \mathscr{O}_{X_{v} n e}=\sigma\left(X_{V^{n e}}, \gamma\right) \cdot \mathscr{O}_{X_{v^{n e}}} \supseteq \sigma\left(X_{v^{n e}}, \bar{\beta}\right)=\sigma\left(X_{v^{n e}}, \Delta_{\bar{\beta}}\right)=\sigma\left(X_{v^{n e}},\left.\Delta_{\gamma}\right|_{X_{v^{n e}}}\right) .
$$

Proof. Obviously, $\sigma\left(X_{V^{n e}}, \gamma\right) \supseteq \sigma\left(X_{V^{n e}}, \beta\right)$. On the other hand, it easily follows that $\sigma\left(X_{V^{n e}}, \beta\right)$ restricts to $\sigma\left(X_{v^{n e}}, \bar{\beta}\right)$ by $F$-adjunction; see for instance [FST11].

The next lemma is a generalization of the fact that for an ideal in a regular ring, $I \subseteq\left(I^{[1 / p]}\right)^{[p]}$ using the ${ }^{[1 / p]}$ notation from [BMS08].

Lemma 3.20. Let $B$ be an $F$-finite regular ring such that $B^{1 / p}$ is a free $B$-module and that $\operatorname{Hom}_{B}\left(B^{1 / p}, B\right) \cong B^{1 / p}$ (for example, this happens if $B$ is local). Further, suppose that $Q$ is a $B^{1 / p^{a}}$-submodule of $B^{1 / p^{a}} \otimes_{B} P$ for some $B$-module $P$, so $Q \subseteq B^{1 / p^{a}} \otimes_{B} P$. Then $Q \subseteq$ $B^{1 / p^{a}} \otimes_{B}\left(\left(\theta \otimes \operatorname{id}_{P}\right)(Q)\right)$, where $\theta \in \operatorname{Hom}_{B}\left(B^{1 / p^{a}}, B\right)$ is the generator.

Proof. The strategy is similar to [BMS08, Proposition 2.5]. By [Kun69, Theorem 2.1], any element of $Q$ can be written as a finite sum $\sum \lambda_{i} \otimes m_{i}$, where $\left\{\lambda_{i}\right\}$ is a basis for $B^{1 / p^{a}}$ over $B$ and $m_{i} \in P$. Now, since $\theta$ is a local generating map of $\operatorname{Hom}_{B}\left(B^{1 / p^{a}}, B\right)$, the projection maps onto the $\lambda_{i}$ are multiples of $\theta$. In other words, for each $\lambda_{i}$, there exists a $u_{i} \in B^{1 / p^{a}}$ such that both $\theta\left(u_{i} \lambda_{i}\right)=1 \in R$ and $\theta\left(u_{i} \lambda_{j}\right)=0 \in R$ for $j \neq i$.

We observe that each $m_{j}$ is then in $\left(\theta \otimes \mathrm{id}_{P}\right)(Q)$ since $\theta\left(u_{j} \sum \lambda_{i} \otimes m_{i}\right)=m_{j}$. It then follows that $\sum \lambda_{i} \otimes m_{i} \in B^{1 / p^{a}} \otimes_{B}\left(\left(\theta \otimes \operatorname{id}_{P}\right)(Q)\right)$.

Lemma 3.21. Suppose that $A=k$ is an $F$-finite field and $K \supseteq k$ is a field extension of $k$ such that $K$ is perfect. Choose a flat map $X=\operatorname{Spec} R \rightarrow V=\operatorname{Spec} A=\operatorname{Spec} k$ of finite type with $X \rightarrow V$ possessing geometrically reduced, G1 and $S 2$ fibers. Additionally, suppose that $\gamma: R^{1 / p^{e}} \rightarrow R$ is any $R$-linear map which is a local generator of $\operatorname{Hom}_{R}\left(R^{1 / p^{e}}, R\right)$ at the generic points of the codimension 1 components of the non-smooth locus of $X$. Then, setting $\Delta_{\gamma}$ as in [BS13, Section 4],

$$
\sigma\left(X, \Delta_{\gamma}\right) \otimes_{k} K \supseteq \sigma\left(X_{K},\left(\Delta_{\gamma}\right) \times_{k} K\right)
$$




\section{$F$-SINGULARITIES IN FAMILIES}

Proof. First, choose $n>0$ such that $\sigma\left(X, \Delta_{\gamma}\right)=\gamma^{n}\left(R^{1 / p^{n e}}\right)$. Since $\operatorname{Hom}_{k}\left(k^{1 / p^{n e}}, k\right)$ is a free $k^{1 / p^{n e}}$-module of rank 1 , by [Kun86, Appendix F] we can factor $\gamma^{n}$ as

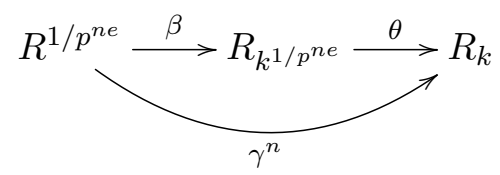

with $\theta$ a local generator of $\mathscr{H} \mathrm{om}_{R_{k}}\left(R_{k^{1 / p^{n e}}}, R_{k}\right)$ and such that $\Delta_{\beta}$ coincides with $\Delta_{\gamma}$. To see this last point, note that if $\gamma$ is a local generator of $\operatorname{Hom}_{R}\left(R^{1 / p^{e}}, R\right)$, then so is $\gamma^{n}$ for $\operatorname{Hom}_{R}\left(R^{1 / p^{n e}}, R\right)$ and hence $\beta$ is also a local generator of $\operatorname{Hom}_{A_{A^{1 / p}} \text { ne }}\left(R^{1 / p^{n e}}, R_{A^{1 / p^{n e}}}\right)$. More generally then, if $\gamma^{n}$ is a local generator pre-multiplied by some $g^{1 / p^{n e}} \in R^{1 / p^{n e}}$ (working in codimension 1 ), then $\beta$ is also a generator times $g^{1 / p^{n e}}$ (possibly times a unit depending on our choice of $\theta$ ). This proves that $\Delta_{\beta}=\Delta_{\gamma}$, as asserted.

We now observe that $\gamma^{n}\left(R^{1 / p^{n e}}\right) \otimes_{k} k^{1 / p^{n e}}=\theta\left(\beta\left(R^{1 / p^{n e}}\right) \otimes_{k} k^{1 / p^{n e}}\right) \supseteq \beta\left(R^{1 / p^{n e}}\right)$ by Lemma 3.20 (here $B=k$ and $P=\beta\left(R^{1 / p^{n e}}\right)$ ). After embedding $k^{1 / p^{n e}} \subseteq K$, we tensor $\beta$ by $K$ to obtain a map

$$
\beta_{K}: R^{1 / p^{n e}} \otimes_{k^{1 / p^{n e}}} K \rightarrow R_{k^{1 / p^{n e}}} \otimes_{k^{1 / p^{n e}}} K .
$$

We immediately see that $\Delta_{\beta_{K}}=\left(\Delta_{\gamma}\right) \times_{k} K$ (again by an argument about local generators). Then, by Lemma 3.20,

$$
\sigma(X, \gamma) \otimes_{k} K \cong \gamma^{n}\left(R^{1 / p^{n e}}\right) \otimes_{k} k^{1 / p^{n e}} \otimes_{k^{1 / p^{n e}}} K \supseteq \beta\left(R^{1 / p^{n e}}\right) \otimes_{k^{1 / p^{n e}}} K \supseteq \sigma\left(X, \beta_{K}\right) .
$$

This completes the proof.

Theorem 3.22. With notation as in Convention 3.14, there exists an $N>0$ such that for all $n>N$, we have $\sigma\left(X_{V^{1 / p^{n e}}}, \Delta_{\gamma}\right) \cdot \mathscr{O}_{X_{s} n e}=\sigma\left(X_{s^{n e}},\left.\left(\Delta_{\gamma}\right)\right|_{X_{s} n e}\right)$ for all perfect points $s \in V$.

Proof. The statement is local, so we may assume that $X$ and $V$ are affine and that $L$ and $M$ are trivial just as above in Lemma 3.18. We know that

$$
\sigma\left(X_{V^{1 / p^{n e}}}, \gamma^{n}\right) \subseteq \operatorname{im}\left(\gamma^{n}\right)=\sigma_{n}(X / V, \phi)
$$

by Lemma 3.12. On the other hand, by Theorem 3.9, we have $\sigma_{n}(X / V, \phi) \cdot \mathscr{O}_{X_{s}}=\sigma\left(X_{s}, \phi_{s}\right)$. Therefore, it is sufficient to show that $\sigma\left(X_{V^{1 / p^{n e}}}, \gamma\right) \cdot \mathscr{O}_{X_{s} n e} \supseteq \sigma\left(X_{s^{n e}}, \psi_{s^{n e}}\right)$.

For this we may assume that $V$ is the spectrum of an $F$-finite, $F$-pure local ring and that Spec $K=s \mapsto V$ has image the closed point Spec $k=v \in V$. We first see that $\sigma\left(X_{\left.V^{n e}, \Delta_{\gamma}\right)}\right.$. $\mathscr{O}_{X_{v} \text { ne }} \supseteq \sigma\left(X_{v^{n e}}, \Delta_{\bar{\beta}}\right)=\sigma\left(X_{v^{n e}},\left.\Delta_{\gamma}\right|_{X_{v} \text { ne }}\right)$ by Lemma 3.19 (and using its notation). On the other hand, by Lemma 3.21,

$$
\sigma\left(X_{v^{n e}},\left.\Delta_{\gamma}\right|_{X_{v}^{n e}}\right) \cdot \mathscr{O}_{X_{s} n e}=\sigma\left(X_{v^{n e}}, \Delta_{\bar{\beta}}\right) \cdot \mathscr{O}_{X_{s} n e} \supseteq \sigma\left(X_{s^{n e}}, \Delta_{\bar{\beta}} \times_{v^{n e}} s^{n e}\right)=\sigma\left(X_{s^{n e}},\left.\Delta_{\gamma}\right|_{X_{s} n e}\right) .
$$

This completes the proof.

Using the same method as for Lemma 3.19, we also obtain the following result, which may also be of independent interest.

Proposition 3.23. Assume that $f: X \rightarrow V$ is a flat, finite-type, reduced, G1 and S2 morphism and that $V$ is regular. Additionally, assume that $\gamma: R^{1 / p^{e}} \rightarrow R$ is an $R$-linear map which is a local generator at the generic points of the codimension 1 components of the non-smooth locus of $f$ for each fiber of $f$. Then, for any $a>0$, we have

$$
\sigma\left(X, \Delta_{\gamma}\right) \otimes_{A} A^{1 / p^{a}} \supseteq \sigma\left(X_{V^{a}}, \Delta_{\gamma} \times_{V} V^{a}\right) .
$$




\section{Z. Patakfalvi, K. Schwede And W. Zhang}

Proof. The statement is local over the base and so we may assume that $K_{V} \sim 0$ and that $A^{1 / p^{e}}$ is a free $A$-module since $V$ is regular [Kun69]. Note, then, that $K_{X / V} \cong K_{X}$. Choose $n e \geqslant a>0$ such that $\sigma\left(X, \Delta_{\gamma}\right)=\gamma^{n}\left(R^{1 / p^{n e}}\right)$. Since $\operatorname{Hom}_{A}\left(A^{1 / p^{a}}, A\right)$ is a free $A^{1 / p^{n e}}$-module of rank 1 , by [Kun86, Appendix F] we can factor $\gamma^{n}$ as

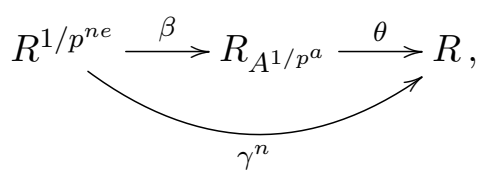

where $\theta$ is a local generator of $\mathscr{H} \mathrm{om}_{R}\left(R_{A^{1 / p^{a}}}, R\right)$. Note that $\beta \in \operatorname{Hom}_{R_{A^{1 / p^{a}}}}\left(R^{1 / p^{n e}}, R_{A^{1 / p^{a}}}\right)$; the latter is identified with

$$
\begin{aligned}
& \mathscr{H} \operatorname{om}_{R_{A^{1 / p^{a}}}}\left(R^{1 / p^{n e}} \otimes_{R_{A^{1 / p^{a}}}}\left(A^{1 / p^{a}} \otimes_{A} \omega_{R / A}\right),\left(A^{1 / p^{a}} \otimes_{A} \omega_{R / A}\right)\right) \\
& \quad \cong \mathscr{H} \operatorname{om}_{R_{A^{1 / p^{a}}}}\left(\left(\mathscr{O}_{X}\left(p^{n e} K_{X / V}\right)\right)^{1 / p^{n e}}, \omega_{R_{A^{1 / p^{a}}} / A^{1 / p^{a}}}\right) \\
& \quad \cong \mathscr{H} \operatorname{om}_{R_{A^{1 / p^{a}}}}\left(\left(\mathscr{O}_{X}\left(p^{n e} K_{X}\right)\right)^{1 / p^{n e}}, \omega_{R_{A^{1 / p^{a}}}}\right) \\
& \quad \cong \mathscr{H} \operatorname{om}_{R^{1 / p^{n e}}}\left(\left(\mathscr{O}_{X}\left(p^{n e} K_{X}\right)\right)^{1 / p^{n e}}, \omega_{R^{1 / p^{n e}}}\right) \\
& \cong\left(\mathscr{O}_{X}\left(\left(1-p^{n e}\right) K_{X}\right)\right)^{1 / p^{n e}} .
\end{aligned}
$$

Note that since the base is regular, all these sheaves are automatically reflexive and so there is no need to double dualize as we did before in Definition 2.8. By dividing by $\left(p^{n e}-1\right)$, we see that $\beta$ coincides with a divisor $\Delta_{\beta}$ such that $\left(1-p^{n e}\right)\left(K_{X}+\Delta_{\beta}\right) \sim 0$. Furthermore, it is easy to see that $\Delta_{\beta}$ coincides with $\Delta_{\gamma}$ just as in Lemma 3.19 since $\theta$ is a local generator.

By composing $\beta$ with a local generating (and surjective) map

$$
R^{1 / p^{n e}} \otimes_{A^{1 / p^{n e}}} A^{1 / p^{n e+a}} \stackrel{\cdots \otimes\left(\Phi_{A}^{a}\right)^{1 / p^{n e}}}{\longrightarrow} R^{1 / p^{n e}},
$$

we then obtain a map

$$
\gamma^{\prime}:\left(R_{A^{1 / p^{a}}}\right)^{1 / p^{n e}} \cong R^{1 / p^{n e}} \otimes_{A^{1 / p^{n e}}} A^{1 / p^{n e+a}} \rightarrow R_{A^{1 / p^{a}}}
$$

satisfying the condition $\Delta_{\gamma^{\prime}}=\Delta_{\gamma} \times_{V} V^{a}$. We also notice that $\gamma^{\prime}$ has the same image as $\beta$.

Now, we observe that $\gamma^{n}\left(R^{1 / p^{n e}}\right) \otimes_{A} A^{1 / p^{a}}=\theta\left(\beta\left(R^{1 / p^{n e}}\right)\right) \otimes_{A} A^{1 / p^{a}} \supseteq \beta\left(R^{1 / p^{n e}}\right)$ by Lemma 3.20, setting $B=A$ and $P=\beta\left(R^{1 / p^{n e}}\right)$. The remainder of the proof is easy since

$$
\begin{aligned}
\sigma\left(X, \Delta_{\gamma}\right) \otimes_{A} A^{1 / p^{a}} & =\gamma^{n}\left(R^{1 / p^{n e}}\right) \otimes_{A} A^{1 / p^{a}} \\
& \supseteq \beta\left(R^{1 / p^{n e}}\right) \\
& =\gamma^{\prime}\left(\left(R_{A^{1 / p^{a}}}\right)^{1 / p^{n e}}\right) \\
& \supseteq \sigma\left(X_{V^{a}}, \Delta_{\gamma^{\prime}}\right) \\
& =\sigma\left(X_{V^{a}}, \Delta_{\gamma} \times_{V} V^{a}\right) .
\end{aligned}
$$

\subsection{Application to sharply $F$-pure singularities and HSL numbers in families}

We observe that Theorem 3.10 has an immediate application. Recall that if $\gamma: L^{1 / p^{e}} \rightarrow R$ is an $R$-linear map, then the HSL number is the first integer $n$ such that $\operatorname{im}\left(\gamma^{n}\right)=\operatorname{im}\left(\gamma^{n+1}\right)=\sigma(R, \gamma)$; cf. [Sha07].

Corollary 3.24 (Uniform behavior of HSL numbers). Given a flat family $f: X \rightarrow V$ over an excellent integral scheme $V$ with a dualizing complex of characteristic $p>0$ and $\phi: L^{1 / p^{e}} \longrightarrow$ 


\section{$F$-SINGULARITIES IN FAMILIES}

$\mathscr{O}_{X_{V}}$ as before, there exists an integer $N>0$ that gives an upper bound on the HSL number of $\left(X_{s}, \phi_{s}: L_{s}^{1 / p^{e}} \rightarrow \mathscr{O}_{X_{s}}\right)$ for every perfect point $s \in V$.

Proof. The statement immediately follows from Theorem 3.9 since, clearly, $\sigma_{m}\left(X_{s} / s, \phi_{s}\right)$ agrees with $\sigma\left(X_{s}, \gamma_{s}=\phi_{s}\right)$ for $m \gg n$.

Now, we study the deformation of sharp F-purity, a question which has been studied before in [SZ09, Has10]. We believe that the hypothesis that $f$ is proper is necessary in Theorem 3.28.

Definition 3.25. Recall that a pair $\left(X, \psi: L^{1 / p^{e}} \rightarrow \mathscr{O}_{X}=R\right)$ is called sharply F-pure if $\sigma(X, \psi)=\mathscr{O}_{X}$. Given $\left(X / S, \phi: L^{1 / p^{e}} \rightarrow R_{A^{1 / p^{e}}}\right)$, we define $(X / S, \phi)$ to be relatively sharply $F$-pure if $\sigma_{n}(X / S, \phi)=R_{A^{1 / p^{n e}}}$ for some $n>0$; cf. [Has10].

LEMmA 3.26. If $\sigma_{n}(X / S, \phi)=R_{A^{1 / p^{n e}}}$ for some $n>0$, then the same holds for all $n>0$.

Proof. Suppose $\sigma_{n}(X / S, \phi)=R_{A^{1 / p^{n e}}}$ for some $n>0$. Choose $m<n$ to start. Then we know $\mathfrak{a}_{m, n} \supseteq \mathfrak{a}_{n, n}=\sigma_{n}(X / S, \phi)$ and so $\mathfrak{a}_{m, n}=R_{A^{1 / p^{n e}}}$. But $\mathfrak{a}_{m, n}=\operatorname{im}\left(\mathfrak{a}_{m} \otimes_{A^{1 / p^{m}}} A^{1 / p^{n}} \rightarrow R_{A^{1 / p^{n}}}\right)$, which is just the extension of $\sigma_{m}(X / S, \phi) \subseteq R_{A^{1 / p^{m e}}}$ to $R_{A^{1 / p^{n e}}}$. Additionally, $R_{A^{1 / p^{m e}}} \subseteq R_{A^{1 / p^{n e}}}$ is an integral extension and hence we must have $\sigma_{m}(X / S, \phi)=R_{A^{1 / p} \text { me }}$ as well.

We finish the proof by showing $\sigma_{2 n}(X / S, \phi)=R_{A^{1 / p^{2 n e}}}$. This follows quickly since $\sigma_{2 n}(X / S, \phi)$ factors as a composition of two surjective maps (as tensor is right exact) as in (2.3) and (2.3).

Remark 3.27. If $S$ is a point, then $(X / S, \phi)$ being relatively sharply $F$-pure is equivalent to $(X / S, \phi)$ being geometrically sharply $F$-pure (in other words, that $\left(X_{t}, \gamma_{t}\right)$ is sharply $F$-pure for every geometric point $t \rightarrow S$, where $\gamma_{t}$ is induced from $\phi_{t}$ as in Section 3.1). To see this, certainly observe that if $(X / S, \phi)$ is relatively sharply $F$-pure, then so is any base change $\left(X_{t} / t, \phi_{t}\right)$. But then, $\left(X_{t}, \gamma\right)$ is sharply $F$-pure by Theorem 3.13. Conversely, if $\left(X_{t}, \gamma_{t}\right)$ is sharply $F$-pure, then certainly $\left(X_{t} / t, \phi_{t}\right)$ is relatively sharply $F$-pure by Lemma 3.12 . But then so is $(X / S, \phi)$ near that $t$ by Nakayama's lemma.

ThEOREM 3.28 (Openness of sharp F-purity). With notation as before, assume that $f: X \rightarrow V$ is proper. Assume that $s \in V$ is a point and that $\left(X_{s} / s, \phi_{s}\right)$ is relatively sharply $F$-pure (in other words, geometrically $F$-pure). Then there exists a dense open set $U \subseteq V$ containing $s$ such that $\left(X_{u} / u, \phi_{u}\right)$ is relatively sharply $F$-pure for all $u \in U$ (in particular, $\left(X_{u}, \phi_{u}\right)$ is sharply $F$-pure for all perfect points $u \in U)$.

Proof. Choose $n \gg 0$ such that $\sigma_{n}\left(X_{s} / s, \phi_{s}\right)=R_{\mathscr{O}_{s}^{1 / p^{n e}}}$ since $\left(X_{s} / s, \phi_{s}\right)$ is sharply F-pure. By Theorem 3.9, we know that $\sigma_{n}(X / V, \phi)=R_{A^{1 / p^{n e}}}$ in a neighborhood $W \subseteq X$ of $X_{s}$. Let $Z=X \backslash W \subseteq X$ be the complement of that neighborhood. Since $f$ is proper, $f(Z)$ is closed and also does not contain $s$. Set $U=V \backslash f(Z)$. Then $\sigma_{n}\left(X_{U} / U, \phi_{U}\right)=R_{\mathscr{O}_{U}^{1 / p^{n e}}}$. It follows from Theorem 3.9 that all the fibers $\left(X_{u} / u, \phi_{u}\right)$ are relatively sharply $F$-pure for perfect $u \in U$, as desired.

We now state the above theorem in the context of divisors.

Corollary 3.29 (Openness of sharp F-purity for divisor pairs). Suppose that $f: X \rightarrow V$ is a proper, $G 1$ and $S 2$ morphism as before and now also suppose that $\Delta$ is a $\mathbb{Q}$-divisor satisfying conditions (a)-(d) from Remark 2.11. Additionally, suppose that $s \in V$ is a perfect point and that $\left(X_{s},\left.\Delta\right|_{X_{s}}\right)$ is sharply $F$-pure (or, more generally, if $s$ is not perfect, that $\left(X_{s^{e}},\left.\Delta\right|_{X_{s} e}\right)$ is sharply $F$-pure for some or all $e>0)$. Then, there exists an open set $U \subseteq V$ containing $s$ such that for all $u \in U$, the pair $\left(X_{u},\left.\Delta\right|_{X_{u}}\right)$ is sharply $F$-pure (respectively, the pair $\left(X_{u^{e}},\left.\Delta\right|_{X_{u^{e}}}\right)$ is sharply $F$-pure for, respectively, some or all $e>0$ and all $u \in U)$. 


\section{Z. Patakfalvi, K. Schwede And W. Zhang}

Proof. Associate to $\Delta$ a relatively divisorial $\phi: L^{1 / p^{e}} \rightarrow R_{A^{1 / p^{e}}}$ as in Remark 2.11. The result then follows immediately from Theorem 3.28 and Corollary 2.20.

\section{Relative test ideals}

In this section, we define a notion of a relative test ideal. We construct it using the following method. We first find some ideal $I$ which when restricted to every geometric fiber is contained in the test ideal of that fiber. We then sum up the images of the $I^{1 / p^{n e}}$ under $\phi^{n}$ (the same method is used to construct the test ideal in the absolute case). This sounds simple enough, but it actually leads to a somewhat disappointing construction. The problem is that the ideal we pick is in no sense canonical and that we do not see a way to make it both canonical and stable under base change. In the case that $\phi$ corresponds to a trivial divisor, we can make our choices canonical by setting $I$ to be the Jacobian ideal (this is done later when we discuss relative $F$-rationality in Section 5), but for more general $\phi$ we do not know of an analogous choice.

We fix the notation of the previous sections (in particular, we fix $\phi: L^{1 / p^{e}} \rightarrow R_{A^{1 / p^{e}}}$ ). We begin by choosing an arbitrary ideal $I \subseteq \mathscr{O}_{X}$. After base change to $k\left(V^{\infty}\right)$ as before, setting $I_{\infty}=I \cdot R_{\infty}$, we can form the following sum:

$$
\tau\left(R_{k\left(V^{\infty}\right)}, \psi_{\infty} I_{\infty}\right):=\sum_{i=0}^{\infty} \psi_{\infty}^{i}\left(\left(I_{\infty} \cdot L_{k\left(V^{\infty}\right)}^{\frac{p^{i e}-1}{p^{e}-1}}\right)^{1 / p^{i e}}\right) .
$$

If it happens that $I_{\infty}$ is contained in the test ideal of $\left(R_{k\left(V^{\infty}\right)}, \phi_{\infty}\right)$ and non-zero on any component of $X_{\infty}$, then we see that the absolute test ideal $\tau\left(R_{k\left(V^{\infty}\right)}, \psi_{\infty}\left(I_{\infty}\right)\right)$ is equal to $\tau\left(R_{k\left(V^{\infty}\right)}, \psi_{\infty}\right)$, for example by [ST12, Section 7].

We can also describe this as follows, as first essentially observed in [Kat08]. Fix ideals

$$
\begin{aligned}
\mathfrak{b}_{0}^{\infty} & :=I_{\infty}=\psi_{\infty}^{0}\left(I_{\infty}\right) \\
\mathfrak{b}_{1}^{\infty} & :=\mathfrak{b}_{0}+\psi_{\infty}\left(\left(\mathfrak{b}_{0}^{\infty} \cdot L_{k\left(V^{\infty}\right)}\right)^{1 / p^{e}}\right)=I_{\infty}+\psi_{\infty}\left(\left(I_{\infty} \cdot L_{k\left(V^{\infty}\right)}\right)^{1 / p^{e}}\right) \\
\mathfrak{b}_{2}^{\infty} & :=\mathfrak{b}_{1}^{\infty}+\psi_{\infty}\left(\left(\mathfrak{b}_{1}^{\infty} \cdot L_{k\left(V^{\infty}\right)}\right)^{1 / p^{e}}\right) \\
& =\mathfrak{b}_{1}^{\infty}+\psi_{\infty}\left(\left(I_{\infty}+\psi_{\infty}\left(\left(I_{\infty} \cdot L_{k\left(V^{\infty}\right)}\right)^{1 / p^{e}}\right) \cdot L_{k\left(V^{\infty}\right)}\right)^{1 / p^{e}}\right) \\
& =\sum_{i=0}^{2} \psi_{\infty}^{i}\left(\left(I_{\infty} \cdot L_{k\left(V^{\infty}\right)}^{\frac{p^{i e}-1}{p^{e}}}\right)^{1 / p^{i e}}\right) \\
\vdots & \mathfrak{b}_{n}^{\infty}:=\mathfrak{b}_{n-1}^{\infty}+\psi_{\infty}\left(\left(\mathfrak{b}_{n-1}^{\infty} \cdot L_{k\left(V^{\infty}\right)}\right)^{1 / p^{e}}\right)=\sum_{i=0}^{n} \psi_{\infty}^{i}\left(\left(I_{\infty} \cdot L_{k\left(V^{\infty}\right)}^{p^{i e}-1}\right)^{1 / p^{i e}}\right) .
\end{aligned}
$$

We notice that this ascending chain stabilizes, say at $t$. Also note that the first time $\mathfrak{b}_{t}^{\infty}=\mathfrak{b}_{t+1}^{\infty}$, we then have $\mathfrak{b}_{t}^{\infty}=\mathfrak{b}_{t+1}^{\infty}=\mathfrak{b}_{t+2}^{\infty}=\cdots$. We fix this integer $t$.

DEFinition 4.1. With notation as above, we define the integer $t$ to be the uniform integer for $\tau$ and $I$ over the generic point of $V$, and, in general, it will be denoted by $n_{\tau(\phi I), k(V)}$. We notice that for any point $\eta \in V$, by base change we can replace $V$ by Spec $k(\eta)$ and form a corresponding integer $n_{\tau\left(\phi_{\eta} I_{\eta}\right), k(\eta)}$. 


\section{$F$-SINGULARITIES IN FAMILIES}

On the other hand, without the passing to $k\left(V^{\infty}\right)$, we have the images

$$
\begin{aligned}
& \mathfrak{b}_{1}:=\operatorname{im}\left(I \otimes_{A} A^{1 / p^{e}} \rightarrow R_{A^{1 / p^{e}}}\right)+\phi\left((I \cdot L)^{1 / p^{e}}\right) \subseteq R_{A^{1 / p^{e}}}, \\
& \mathfrak{b}_{2}:=\sum_{i=0}^{2} \operatorname{im}\left(\phi^{i}\left(\left(I \cdot L^{\frac{p^{i e}-1}{p^{e}-1}}\right)^{1 / p^{i e}}\right) \otimes_{A^{1 / p^{i e}}} A^{1 / p^{2 e}} \rightarrow R_{A^{1 / p^{2 e}}}\right) \subseteq R_{A^{1 / p^{2 e}}}, \\
& \vdots \\
& \mathfrak{b}_{n}:=\sum_{i=0}^{n} \operatorname{im}\left(\phi^{i}\left(\left(I \cdot L^{\frac{p^{i e}-1}{p^{e}-1}}\right)^{1 / p^{i e}}\right) \otimes_{A^{1 / p^{i e}}} A^{1 / p^{n e}} \rightarrow R_{A^{1 / p^{n e}}}\right) \subseteq R_{A^{1 / p^{n e}}},
\end{aligned}
$$

Notice that $\operatorname{im}\left(\mathfrak{b}_{1} \otimes_{A^{1 / p^{e}}} A^{1 / p^{2 e}} \rightarrow R_{A^{1 / p^{2 e}}}\right) \subseteq \mathfrak{b}_{2}$ and, more generally, that $\operatorname{im}\left(\mathfrak{b}_{i} \otimes_{A^{1 / p^{i e}}}\right.$ $\left.A^{1 / p^{j e}} \rightarrow R_{A^{1 / p^{n e}}}\right) \subseteq \mathfrak{b}_{j}$ for $j>i$. Also observe that this is the opposite containment compared to what we had in Section 3.

By the same argument as in Section 3, we know that there exists an open set $U \subseteq V$ with $W=f^{-1}(U)$ such that

$$
\left.\operatorname{im}\left(\mathfrak{b}_{t} \otimes_{A^{1 / p^{t e}}} A^{1 / p^{(t+1) e}} \rightarrow R_{A^{1 / p}(t+1) e}\right)\right|_{W}=\left.\mathfrak{b}_{t+1}\right|_{W}
$$

As before in Proposition 3.3, we claim that the following holds.

LEMMA 4.2. With notation as above, $\left.\operatorname{im}\left(\mathfrak{b}_{n} \otimes_{A^{1 / p^{n e}}} A^{1 / p^{(n+1) e}} \rightarrow R_{A^{1 / p^{(n+1) e}}}\right)\right|_{W}=\left.\mathfrak{b}_{n+1}\right|_{W}$ for all $n \geqslant t$.

Proof. Replacing $V$ by $U$ and $X$ by $W$, we may assume

$$
\mathfrak{b}_{t+1}=\operatorname{im}\left(\mathfrak{b}_{t} \otimes_{A^{1 / p^{t e}}} A^{1 / p^{(t+1) e}} \rightarrow R_{A^{1 / p^{(t+1) e}}}\right) .
$$

By induction on $n$, it suffices to show that if $\mathfrak{b}_{n+1}=\operatorname{im}\left(\mathfrak{b}_{n} \otimes_{A^{1 / p^{n e}}} A^{1 / p^{(n+1) e}} \rightarrow R_{A^{1 / p}(n+1) e}\right)$, then $\mathfrak{b}_{n+2}=\operatorname{im}\left(\mathfrak{b}_{n+1} \otimes_{A^{1 / p^{(n+1) e}}} A^{1 / p^{(n+2) e}} \rightarrow R_{A^{1 / p}}(n+2) e\right)$. Note that $\mathfrak{b}_{n+1}=\operatorname{im}\left(\mathfrak{b}_{n} \otimes_{A^{1 / p^{n e}}}\right.$ $\left.A^{1 / p^{(n+1) e}} \rightarrow R_{A^{1 / p^{(n+1) e}}}\right)$ if and only if

$$
\begin{aligned}
\operatorname{im}\left(\phi^{n+1}\left(\left(I \cdot L^{\frac{p^{(n+1) e}-1}{p^{e}-1}}\right)^{1 / p^{(n+1) e}}\right) \rightarrow R_{A^{1 / p^{(n+1) e}}}\right) \\
\subseteq \sum_{i=0}^{n} \operatorname{im}\left(\phi^{i}\left(\left(I \cdot L^{\frac{p^{i e}-1}{p^{e}-1}}\right)^{1 / p^{i e}}\right) \otimes_{A^{1 / p^{i e}}} A^{1 / p^{(n+1) e}} \rightarrow R_{A^{1 / p^{(n+1) e}}}\right) .
\end{aligned}
$$

Tensoring with $L$ over $R$, taking $p^{e}$ th roots, and applying $\phi$ yields

$$
\begin{aligned}
\operatorname{im}\left(\phi^{n+2}\left(\left(I \cdot L^{\frac{p^{(n+2) e}-1}{p^{e}-1}}\right)^{1 / p^{(n+2) e}}\right) \rightarrow R_{A^{1 / p^{(n+2) e}}}\right) \\
\subseteq \sum_{i=1}^{n+1} \operatorname{im}\left(\phi^{i}\left(\left(I \cdot L^{\frac{p^{i e}-1}{p^{e}-1}}\right)^{1 / p^{i e}}\right) \otimes_{A^{1 / p^{i e}}} A^{1 / p^{(n+2) e}} \rightarrow R_{A^{1 / p^{(n+2) e}}}\right) .
\end{aligned}
$$

Hence,

This finishes the proof.

$$
\mathfrak{b}_{n+2}=\operatorname{im}\left(\mathfrak{b}_{n+1} \otimes_{A^{1 / p^{(n+1) e}}} A^{1 / p^{(n+2) e}} \rightarrow R_{A^{1 / p^{(n+2) e}}}\right) .
$$

As before, we define relative test ideals as follows. 


\section{Z. Patakfalvi, K. Schwede And W. Zhang}

Definition 4.3. The $n$th limiting relative test ideal with respect to $I$ (of the pair $(X / V, \phi)$ ) is defined to be $\mathfrak{b}_{n} \subseteq R_{A^{1 / p^{n e}}}$ and is denoted by $\tau_{n}(X / V, \phi I)$.

Before proceeding to various properties of relative test ideals, we give one example of relative test ideals.

EXAMPLE 4.4 (Example 3.7, revisited). Fix $k$ to be an algebraically closed field of characteristic $p>2$, set $A=k[t]$, and set $R=k[x, t]$ with the obvious map $X=\operatorname{Spec}(R) \rightarrow V=\operatorname{Spec}(A)$. Let $\phi: R^{1 / p}=k\left[x^{1 / p}, t^{1 / p}\right] \rightarrow R_{A^{1 / p}}=k\left[x, t^{1 / p}\right]$ be the composition of the local generator $\beta \in \operatorname{Hom}_{R_{A^{1 / p}}}\left(R^{1 / p}, R_{A^{1 / p}}\right)$ with pre-multiplication by $\left(x^{p^{2}}+t\right)^{1 / p}=f^{1 / p}$. Set $I=\left\langle x^{p}+t\right\rangle$. Then

$$
\begin{aligned}
\mathfrak{b}_{1} & =\operatorname{im}\left(I \otimes_{A} A^{1 / p} \rightarrow R_{A^{1 / p}}\right)+\phi\left(I^{1 / p}\right)=\left\langle x^{p}+t\right\rangle+\left\langle\left(x^{p}+t^{1 / p}\right)\left(x+t^{1 / p}\right)\right\rangle \\
& =\left\langle x^{p}+t,\left(x^{p}+t^{1 / p}\right)\left(x+t^{1 / p}\right)\right\rangle .
\end{aligned}
$$

To calculate $\mathfrak{b}_{2}$, it suffices to calculate

$$
\operatorname{im}\left(\phi^{2}\left(I^{1 / p^{2}}\right)\right)=\operatorname{im}\left(\beta^{2}\left(\left(x^{p^{2}}+t\right)^{\frac{1+p}{p^{2}}} I^{1 / p^{2}}\right)\right) .
$$

Since $\left(x^{p^{2}}+t\right)^{(1+p) / p^{2}} \in R_{A^{1 / p^{2}}}$, we can see that $\operatorname{im}\left(\phi^{2}\left(I^{1 / p^{2}}\right)\right) \subseteq\left\langle\left(x^{p^{2}}+t\right)^{(1+p) / p^{2}}\right\rangle$. On the other hand, it is straightforward to check that

$$
\beta^{2}\left(\left(x^{p^{2}}+t\right)^{\frac{1+p}{p^{2}}} x^{\frac{p^{2}-p-1}{p^{2}}}\left(x^{p}+t\right)^{1 / p^{2}}\right)=\left(x^{p^{2}}+t\right)^{\frac{1+p}{p^{2}}}=\left(x+t^{1 / p^{2}}\right)\left(x^{p}+t^{1 / p}\right) .
$$

Therefore,

$$
\mathfrak{b}_{2}=\left\langle x^{p}+t,\left(x^{p}+t^{1 / p}\right)\left(x+t^{1 / p}\right),\left(x+t^{1 / p^{2}}\right)\left(x^{p}+t^{1 / p}\right)\right\rangle .
$$

For each $i \geqslant 2$, to calculate $\mathfrak{b}_{i+1}$, it suffices to calculate $\operatorname{im}\left(\beta^{i+1}\left(\left(x^{p^{2}}+t\right)^{\frac{1+p+\cdots+p^{i}}{p^{i+1}}} I^{1 / p^{i+1}}\right)\right)$. It is straightforward to check that it is contained in $\left\langle\left(x^{p}+t^{1 / p}\right)\left(x+t^{1 / p^{2}}\right)\right\rangle$ and hence is contained in $\mathfrak{b}_{2}$. Therefore, $\mathfrak{b}_{i+1}=\mathfrak{b}_{2}$ as well. So we have

$$
\mathfrak{b}_{n}= \begin{cases}\left\langle x^{p}+t,\left(x^{p}+t^{1 / p}\right)\left(x+t^{1 / p}\right)\right\rangle & \text { when } n=1 \\ \mathfrak{b}_{2}=\left\langle x^{p}+t,\left(x^{p}+t^{1 / p}\right)\left(x+t^{1 / p}\right),\left(x+t^{1 / p^{2}}\right)\left(x^{p}+t^{1 / p}\right)\right\rangle & \text { when } n \geqslant 2 .\end{cases}
$$

We now prove a base change statement for relative test ideals. Compare with Proposition 3.8. As before, we fix $q_{i}: X \times_{V} T^{i} \rightarrow X \times_{V} V^{i}$ to be the natural map.

Theorem 4.5 (Relative test ideals and base change). Fix $g: T \rightarrow V$ to be any morphism with $T$ excellent, integral, and admitting a dualizing complex. Then

$$
\operatorname{im}\left(\left(q_{n e}\right)^{*} \tau_{n}(X / V, \phi I) \hookrightarrow \mathscr{O}_{X_{T^{n e}}}\right):=\tau_{n}(X / V, \phi I) \cdot \mathscr{O}_{X_{T^{n e}}}=\tau_{n}\left(X_{T} / T, \phi_{T} I_{T}\right) .
$$

Furthermore, if $U=U_{n}$ satisfies the condition of Lemma 4.2, then $W=g^{-1}(U) \subseteq T$ satisfies the same condition for $\tau_{n}\left(X_{T} / T, \phi_{T} I_{T}\right)$.

Proof. We write $B=\mathscr{O}_{T}$ and work locally. By construction and right exactness of tensor,

$$
\begin{aligned}
\tau_{n}(X / V, \phi) \cdot \mathscr{O}_{X_{T^{n e}}} & =\mathfrak{b}_{n} \otimes_{A^{1 / p^{n e}}} B^{1 / p^{n e}} \\
& =\sum_{i=0}^{n}\left(\phi^{i}\left(\left(I \cdot L^{\frac{p^{i e}-1}{p^{e}-1}}\right)^{1 / p^{i e}}\right) \otimes_{A^{1 / p^{i e}}} B^{1 / p^{n e}}\right) \subseteq R_{B^{1 / p^{n e}}} .
\end{aligned}
$$




\section{$F$-SINGULARITIES IN FAMILIES}

But, by Lemma 2.15,

$$
\begin{aligned}
\sum_{i=0}^{n}\left(\phi^{i}\left(\left(I \cdot L^{\frac{p^{i e}-1}{p^{e}-1}}\right)^{1 / p^{i e}}\right) \otimes_{A^{1 / p^{i e}}} B^{1 / p^{n e}}\right) & =\sum_{i=0}^{n}\left(\phi_{T}^{i}\left(\left(I_{T} \cdot L_{T}^{\frac{p^{i e}-1}{p^{e}-1}}\right)^{1 / p^{i e}}\right) \otimes_{B^{1 / p^{i e}}} B^{1 / p^{n e}}\right) \\
& =\tau_{n}\left(X_{T} / T, \phi_{T} I_{T}\right)
\end{aligned}
$$

which completes the proof.

THEOREM 4.6 (Restriction of $\tau_{n}$ to fibers). With notation as above, there exists an integer $N \geqslant 0$ such that for all points $s \in V$, we have $N \geqslant n_{\tau\left(\phi_{s} I\right), k(s)}$. In other words, we have both that $\tau_{n}(X / V, \phi) \cdot \mathscr{O}_{X_{s} n e}=\tau_{n}\left(X_{s} / s, \phi_{s}\right)$ (which always holds) and that for all $m \geqslant n \geqslant N$,

$$
\tau_{n}(X / V, \phi I) \otimes_{V^{n}} k(s)^{1 / p^{m}}=\tau_{m}\left(X_{s} / s, \phi_{s} I_{s}\right) .
$$

Proof. The idea is the same as for Theorem 3.9 and we only sketch it here. We stratify $V$ as follows. The result holds on a dense open subset of $U_{0} \subseteq V$. Let $V_{1}^{\prime}$ be the complement, and let $V_{1}$ denote the regular locus of $V_{1}^{\prime}$. Base change to $V_{1}$, and then repeat. This procedure stops after finitely many iterations by Noetherian induction, and we choose an $N$ that works for them all.

We now construct $I \subseteq R$ whose restriction is contained in the test ideal of every geometric fiber.

Proposition 4.7 (Existence of relative test elements). With notation as above, suppose additionally that $f: X \rightarrow V$ is relatively $G 1$ and $S 2$. Then, there exists an ideal $I \subset \mathscr{O}_{X}$ such that for every point $s \in V$ and every perfect extension $K \supseteq k(s)$, we have $I_{K} \subseteq \tau\left(X_{K}, \psi_{K}\right)$, where again $\psi_{K}$ is simply $\phi_{K}$ interpreted as in [BS13]. Additionally, we can assume $I=\mathscr{O}_{X}$ at all points of $X$ such that both $X / V$ is smooth and $\phi$ locally generates $\mathscr{H}_{\operatorname{om}_{\mathscr{O}_{X}}}\left(L^{1 / p^{e}}, R_{A^{1 / p^{e}}}\right)$. Furthermore, if $V$ is strongly $F$-regular and quasi-Gorenstein, then $I$ can additionally be chosen such that $I_{V^{e}}$ is within the absolute test ideal of $\left(X_{V^{e}}, \gamma\right)$ (where $\gamma$ is as in Section 3.1).

We caution the reader that it is possible that $\psi_{s}$ (and thus $\psi_{K}$ ) could be the zero map, and hence $I_{K}$ the zero ideal.

Proof. First, let $Z_{1} \subseteq X$ denote the locus where $\phi R^{1 / p^{e}} \subseteq \mathscr{H} \operatorname{om}_{R_{A^{1 / p^{e}}}}\left(L^{1 / p^{e}}, R_{A^{1 / p^{e}}}\right)$ is not an isomorphism. Additionally, let $Z_{2}$ denote the locus where $X$ is not smooth over $V$. Set $Z=Z_{1} \cup Z_{2}$. Set $W=X \backslash Z$ (and note that we can also view this as a subset of $X_{V}$ for any $e$ ) and notice that on $W$, we know that $\phi$ can be identified with the trace map up to multiplication by a unit.

Since $\left.f\right|_{W}$ is smooth, we observe that $\phi$ (which is identified with the trace map) is surjective when restricted to $W$ by Corollary 2.5. Now, choose an exponent $m>0$ such that

$$
I_{Z}^{m} \cdot R_{A^{1 / p^{e}}} \subseteq \mathfrak{a}_{1,1}=\sigma_{1}(X / S, \phi) .
$$

Now, choose an integer $l>0$ such that $I_{Z}^{m l}$ is locally generated by cubes of elements of $I_{Z}^{m}$ (this only depends on the number of local generators of $I_{Z}$ ). Then the formation of $I_{Z}^{m l}$ is obviously compatible with base change (in that the extension of $I_{Z}^{m l}$ to the base change will also satisfy the same containment condition.). Thus, set $I=I_{Z}^{m l}$. The first result follows immediately from [Hoc07, Theorem on p. 90] or [Sch11, proof of Proposition 3.21].

For the second result, we notice that $\gamma$ locally generates $\mathscr{H} \mathrm{om}_{R_{A^{1 / p}}}\left(N^{1 / p^{e}}, R_{A^{1 / p^{e}}}\right)$ and that $I_{Z}^{m}$ is also in the image of $\gamma$. Therefore, $I^{m l}$ works by the above references. 


\section{Z. Patakfalvi, K. Schwede And W. Zhang}

Therefore we obtain the following result.

COROLlary 4.8. Using the notation of Theorem 4.6, assume further that I satisfies the condition of Proposition 4.7. If $s$ is a perfect point, then for all $n \geqslant N$ as in Theorem 3.9,

$$
\tau_{n}(X / V, \phi I) \otimes_{V^{n}} k(s)^{1 / p^{m}}=\tau\left(X_{s}, \psi_{s}\right),
$$

where $\psi_{s}$ is $\phi_{s}$ interpreted as a $p^{-e}$-linear map.

Proof. Simply observe that, in general, the ascending chain $\tau_{j}\left(X_{s} / s, \phi_{s} I_{s}\right) \subseteq \tau_{j+1}\left(X_{s} / s, \phi_{s} I_{s}\right) \subseteq$ … stabilizes to $\tau\left(X_{s}, \psi_{s}\right)$. Furthermore, by Theorem 4.6, this ascending chain stabilizes.

COROLlary 4.9. Using the notation of Theorem 4.6, assume further that I satisfies the condition of Proposition 4.7. If the perfect closure of the generic fiber of $f: X \rightarrow V$ is strongly $F$-regular, that is, $\tau\left(R_{k\left(V^{\infty}\right)}, \psi_{\infty} I_{\infty}\right)=R_{k\left(V^{\infty}\right)}$, then there exists an open subset $U \subseteq V$ such that $\left(X_{s}, \psi_{s}\right)$ is also strongly $F$-regular for each perfect point $s \in U$.

Proof. It follows immediately from the proof of Proposition 3.3 and Corollary 4.8.

\subsection{Relative test ideals versus absolute test ideals}

We make the following assumption.

Convention 4.10. For the rest of Section 4.1, we assume that $V$ is regular and $F$-finite.

It is natural to try to relate the relative test ideal $\tau_{n}(X / V, \phi I)$ to the absolute test ideal $\tau\left(X_{V^{n e}}, \gamma^{n}\right)$ as in Section 3.1. Indeed, if we construct $I$ as we did in the proof of Proposition 4.7, then it follows easily that $I \cdot R_{A^{1 / p^{n e}}} \subseteq \tau\left(X_{V^{n e}}, \gamma^{n}\right)$. Thus, fix such an $I$.

We now recall the following diagram from Lemma 3.12:

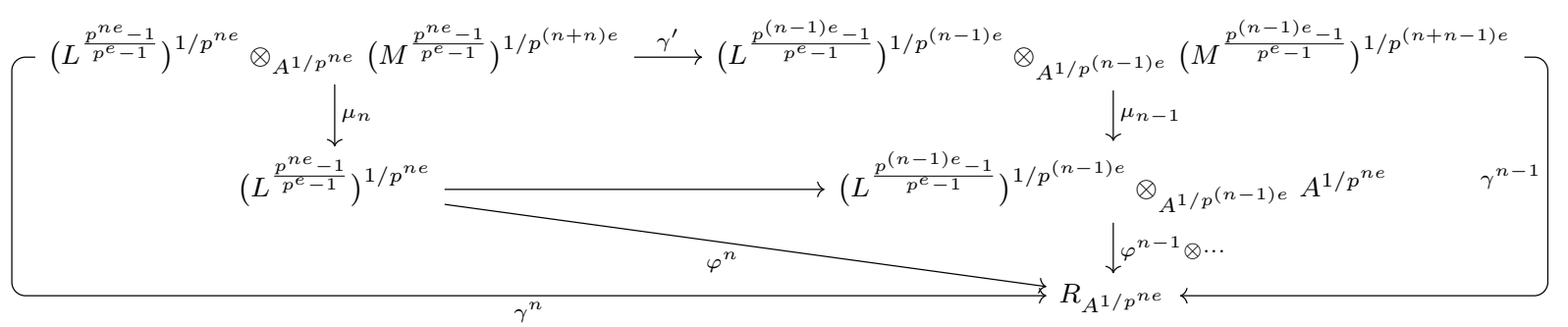

We let $I_{A^{1 / p^{k e}}}^{1 / p^{j e}}$ denote the extension of $I^{1 / p^{j e}}$ to $R_{A^{1 / p^{k e}}}^{1 / p^{j e}}$. Then notice that $\mu_{j}$ sends $I_{A^{1 / p}}^{1 / p^{j e}}$ (times the domain of $\mu_{j}$ ) back onto $I^{1 / p^{j e}}$ since each $\mu_{j}$ is surjective.

We then observe that the following holds.

LEMmA 4.11. With notation as above and assuming Convention 4.10, we have $\tau_{n}(X / V, \phi I) \subseteq$ $\tau\left(X_{V^{n e}}, \gamma\right)$.

Proof. We know that $\tau_{n}(X / V, \phi I)$ is the sum

$$
\sum_{j=0}^{n}\left(\phi^{j} \otimes_{A^{1 / p^{j e}}} A^{1 / p^{n e}}\right)\left(I^{1 / p^{j e}} \cdot\left(L^{\frac{p^{j e}-1}{p^{e}-1}}\right)^{1 / p^{j e}} \otimes_{A^{1 / p^{j e}}} A^{1 / p^{n e}}\right) .
$$

On the other hand, $\tau(X, \gamma)$ is the sum

$$
\sum_{j=0}^{\infty} \gamma^{j}\left(I_{A^{1 / p^{(j+n) e}}}^{1 / p^{j e}} \cdot\left(L^{\frac{p^{j e}-1}{p^{e}-1}}\right)^{1 / p^{j e}} \otimes_{A^{1 / p^{j e}}}\left(M^{\frac{p^{j e}-1}{p^{e}-1}}\right)^{1 / p^{(j+n) e}}\right) .
$$




\section{$F$-SINGULARITIES IN FAMILIES}

By our above observations about the surjectivity of the $\mu_{j}$ above, the sum of the 0th through $j$ th terms of (4.4) is equal to the sum (4.3). The result follows.

Additionally, at least over an open subset of the base, the relative test ideal and absolute test ideal actually agree; cf. Theorem 3.13.

Theorem 4.12. With notation as above and assuming Convention 4.10 , choose $n>t=$ $n_{\tau(\phi I), k(V)}$. Then there exists a dense open set $U \subseteq V \cong V^{e}$ with $W=f^{-1}(U) \subseteq X$ such that $\left.\tau\left(X_{V^{1 / p^{n e}}}, \gamma\right)\right|_{W}=\left.\tau_{n}(X / V, \phi I)\right|_{W}$. Furthermore, shrinking $U$ further if necessary and possibly increasing $n$, we can require for all perfect points $u \in U$ that

$$
\tau\left(X_{V^{1 / p^{n e}}}, \gamma\right) \cdot \mathscr{O}_{X_{u}}=\tau_{n}\left(X_{u} / u, \phi_{u} I_{u}\right)=\tau\left(X_{u}, \psi_{u}\right),
$$

where again $\psi_{u}$ is $\phi_{u}$ viewed as a $p^{-e}$-linear map.

Proof. First, we observe that by taking $U$ as in (4.2), we can assume $\mathfrak{b}_{n-1, n}=\mathfrak{b}_{n, n}$. But by the diagram above, we see that the $(n-1)$ st partial sum defining $\tau\left(X_{V^{1 / p^{n e}}}, \gamma\right)$ in $(4.4)$ is equal to $\mathfrak{b}_{n-1, n}$. Likewise, the $n$th partial sum is equal to $\mathfrak{b}_{n, n}$. However, once two adjacent partial sums defining $\tau\left(X_{V^{1 / p^{n e}}}, \gamma\right)$ coincide, the sum stabilizes for further powers by the computation we made when defining the $\mathfrak{b}_{n}^{\infty}$ in (4.1). The second statement follows from the first and from Theorem 4.5; cf. the argument of Corollary 4.8.

When dealing with relatively non- $F$-pure ideals $\sigma$, we actually obtained the above restriction theorem without shrinking $X$ to $U$ in Corollary 3.22. The difference is that for $\sigma$, we have the easy containment $\sigma\left(X_{V^{1 / p^{n e}}}, \gamma^{n}\right) \subseteq \sigma_{n}(X / V, \phi)$ by Lemma 3.12. For $\tau$, however, the easy containment is reversed. This leads us to the following question.

QUESTION 4.13. Is it true that $\tau\left(X_{V^{1 / p^{n e}}}, \gamma\right) \cdot \mathscr{O}_{X_{s} n e}=\tau\left(X_{s}, \psi_{s}\right)$ for all perfect points $s \in V$, at least when $n \gg 0$ ?

Rephrasing Theorem 4.12 for divisors, we obtain the following statement.

Corollary 4.14. Suppose that $f: X \rightarrow V$ is a flat finite map to an excellent regular scheme $V$. Additionally, suppose that $f$ is relatively $G 1$ and $S 2$ and $I$ is chosen as in Proposition $4.7+$. Choose $\Delta$ satisfying conditions (a)-(d) of Remark 2.11. Then, there exists an open dense set $U \subseteq V$ such that

$$
\tau\left(X_{V^{1 / p^{n e}}}, \Delta\right) \cdot \mathscr{O}_{X_{u^{1 / p^{n e}}}}=\tau\left(X_{u},\left.\Delta\right|_{X_{u}}\right)
$$

for all perfect points $u \in U$.

Proof. Using Lemmas 2.21 and 3.15, we see that $\Delta$ corresponds to some $\phi: L^{1 / p^{e}} \rightarrow R_{A^{1 / p^{e}}}$. Thus, we simply apply Corollary 2.20 and Theorem 4.12.

\subsection{Applications to strong $F$-regularity and divisor pairs}

Definition 4.15. Recall that a pair $\left(X, \psi: L^{1 / p^{e}} \rightarrow \mathscr{O}_{X}=R\right)$ is called strongly $F$-regular if $\tau(X, \psi)=\mathscr{O}_{X}$. Given $\left(X / S, \phi: L^{1 / p^{e}} \rightarrow R_{A^{1 / p^{e}}}\right)$ and some $I \subseteq R$ satisfying the condition of Proposition 4.7 , we say that $(X / S, \phi I)$ is relatively strongly $F$-regular if we have $\tau_{n}(X / S, \phi I)=$ $R_{A^{1 / p^{n e}}}$ for some $n>0$ (equivalently, all $n \gg 0$ since the $\tau_{n}$ ascend); cf. [Has10].

Remark 4.16. Suppose that $S$ is a point and $I$ is chosen as in Proposition 4.7 ; then $(X / S, \phi I)$ is relatively strongly $F$-regular if and only if it is geometrically strongly $F$-regular (that is, $\left(X_{t}, \gamma_{t}\right)$ is strongly $F$-regular for every point $t \rightarrow S$ ). The argument is the same as in Remark 3.27. 


\section{Z. Patakfalvi, K. Schwede And W. Zhang}

The same ideas imply that the strongly $F$-regular locus of a proper map is open.

Corollary 4.17 (Openness of the strongly F-regular locus). With notation as before, assume additionally that $f: X \rightarrow V$ is proper and that $I$ satisfies the condition of Proposition 4.7. Assume that $s \in V$ is a point and that $\left(X_{s} / s, \phi_{s} I_{s}\right)$ is relatively strongly $F$-regular (for example, if $s$ is a perfect point, this just means that it is strongly $F$-regular and independent of $I)$. Then, there exists a dense open set $U \subseteq V$ containing $s$ such that $\left(X_{u} / u, \phi_{u} I_{u}\right)$ is relatively strongly $F$-regular for all $u \in U$ (in particular, $\left(X_{u}, \phi_{u}\right)$ is strongly $F$-regular for all perfect points $u \in U$ ).

Proof. Choose $n \gg 0$ such that $\tau_{n}\left(X_{s} / s, \phi_{s} I_{s}\right)=R_{\mathscr{O}_{s}^{1 / p^{n e}}}$, since $\left(X_{s} / s, \phi_{s} I_{s}\right)$ is strongly $F$ regular. By Theorem 4.6, we know that $\tau_{n}(X / V, \phi I)=R_{A^{1 / p^{n e}}}$ in a neighborhood $W \subseteq X$ of $X_{s}$. Let $Z=X \backslash W \subseteq X$ be the complement of that neighborhood. Since $f$ is proper, $f(Z)$ is closed, and also does not contain $s$. Set $U=V \backslash f(Z)$. Then $\tau_{n}\left(X_{U} / U,\left.\phi_{U} I\right|_{U}\right)=R_{\mathscr{O}_{U}^{1 / p^{n e}}}$. It follows from Theorem 4.6 that all the fibers $\left(X_{u} / u, \phi_{u} I_{u}\right)$ are relatively strongly $F$-regular, as desired.

At least for proper maps, we also obtain that the definition of relative strongly $F$-regularity (Definition 4.15) is independent of the choice of $I$.

Lemma 4.18. Suppose that $f: X \rightarrow V$ is proper and that $(X / V, \phi I)$ is relatively strongly $F$ regular for some $I$ satisfying the condition of Proposition 4.7. Then for all $J \subseteq R$ such that $J_{s}$ is non-zero on every component of every fiber $X_{s}$, we have that $\tau_{n}(X / S, \phi J)=R_{A^{1 / p^{n e}}}$ for some $n>0$ (equivalently, all $n \gg 0$ ).

In particular, in Definition 4.15 , it would be equivalent to require $\tau_{n}(X / S, \phi J)=R_{A^{1 / p^{n e}}}$ for all such $J$ and some $n$.

Proof. Choose a closed point $s \in V$ and a perfect extension $K \supseteq k(s)$. Then $\tau_{m}\left(X_{K} / K, \phi_{K} I_{K}\right)=$ $R_{K^{1 / p^{n e}}}$ for some $m>0$ by Theorem 4.6. But, since $K$ is a perfect field extension, this implies that $\tau_{m}\left(X_{K}, \psi_{K} I_{K}\right)=R_{K^{1 / p} \text { me }}$ as well. On the other hand, since $\left(X_{K}, \psi_{K}\right)$ is strongly $F$-regular, we see that $\tau_{n}\left(X_{K} / K, \phi_{K} J_{K}\right)=R_{K^{1 / p^{n e}}}$ for some $n>0$. Hence, since $k(s) \subseteq K$ is faithfully flat,

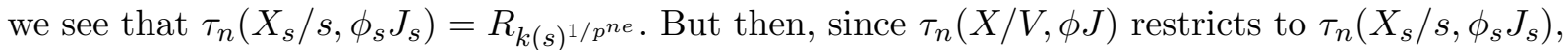
we observe that $\tau_{n}(X / V, \phi J)=R_{A^{1 / p} \text { ne }}$ at least in a neighborhood of $s$, using that $f$ is proper and the same argument we made in Corollary 4.17. But we can find such an $n$ for every $s \in V$, and so the lemma holds by the quasi-compactness of $V$.

We now state our result in the divisorial case.

Corollary 4.19. With notation as above, suppose that $f: X \rightarrow V$ is a proper map and that $\Delta$ is a $\mathbb{Q}$-divisor satisfying conditions (a)-(d) ${ }^{8}$ of Remark 2.11. Additionally, suppose that for some perfect point $s \in V$, the fiber $\left(X_{s},\left.\Delta\right|_{X_{s}}\right)$ is strongly $F$-regular. Then there exists a dense open set $U \subseteq V$ containing $s$ such that $\left(X_{u},\left.\Delta\right|_{X_{u}}\right)$ is strongly $F$-regular for all perfect $u \in U$.

Proof. Using Remark 2.11, we construct a relatively divisorial $\phi: L^{1 / p^{e}} \rightarrow R_{A^{1 / p^{e}}}$ corresponding to $\Delta$. Now, choose $I$ satisfying the condition of Proposition 4.7. It follows that $\left(X_{s}, \psi_{s}\right)$ is strongly $F$-regular and hence that $\left(X_{s} / s, \phi_{s} I_{s}\right)$ is relatively strongly $F$-regular since $s$ is a perfect point. Then Corollaries 4.17 and 2.20 complete the proof.

By a perturbation trick we can also handle the case that the index of $K_{X}+\Delta$ is divisible by $p>0$, at least over curves.

${ }^{8}$ For example, these conditions hold if $V$ is regular, $f: X \rightarrow V$ is geometrically normal, $K_{X}+\Delta$ is $\mathbb{Q}$-Cartier with index not divisible by $p$, and $\Delta$ does not contain any fiber of $f$ in its support. 


\section{$F$-SINGULARITIES IN FAMILIES}

Corollary 4.20. With notation as above, suppose that $f: X \rightarrow V$ is a projective map to a regular 1-dimensional base and that $\Delta$ is a $\mathbb{Q}$-divisor satisfying the following four conditions:

(a') We have $\Delta^{\prime}=\frac{1}{m} D$ for some Weil divisor $D$.

(b) The divisor $D$ is a Weil divisor on $X$ which is Cartier in codimension 1 and Cartier at every codimension 1 point of every fiber.

(c) The divisor $D$ is trivial along the codimension 1 components of the non-smooth locus of $X \rightarrow V$ and the codimension 1 components of the non-smooth locus of every fiber.

(d') We have $l / m \in \mathbb{Z}$, and $\left(\omega_{X / V}^{l} \otimes \mathscr{O}_{X}\left(l \Delta^{\prime}\right)\right)^{* *}$ is a line bundle.

Additionally, suppose that for some perfect point $s \in V$, the fiber $\left(X_{s},\left.\Delta\right|_{X_{s}}\right)$ is strongly $F$ regular. Then there exists a dense open set $U \subseteq V$ containing $s$ such that $\left(X_{u},\left.\Delta\right|_{X_{u}}\right)$ is strongly $F$-regular for all perfect $u \in U$.

Proof. We may certainly suppose that $V$ is affine. Since the fiber $X_{s}$ is normal, and hence geometrically normal, we may also assume that the nearby fibers satisfy the same condition (using that $f$ is proper). Thus, we may assume that all the fibers are geometrically normal and in fact that the map $f: X \rightarrow V$ is geometrically integral. Without loss of generality, by base change we can assume that $V$ is normal and hence $X$ is normal itself. Thus, we may assume that $X$ is normal.

Now, since the base is 1-dimensional, we claim that we can assume that $K_{X / V}$ does not contain any fiber. The only fiber we must worry about is $X_{s}$ (as the others can be handled by shrinking $V$ ). We argue as follows: First, note that $K_{X / V}$ can be viewed as an honest Weil divisor since we already assumed that $X$ is normal. On the other hand, each fiber is a Cartier divisor. Hence, if $K_{X / V}$ is non-trivial along the generic point of $X_{s}$, by twisting by the pullback of $s$, we can assume that $K_{X / V}$ does not contain $X_{s}$.

Write $l \Delta^{\prime}=c p^{e_{0}} \Delta^{\prime}$, where $p$ does not divide $c$. Let $E$ be an effective Cartier divisor on $X$ not containing any fiber such that $K_{X / V}+E$ is effective. We also observe that $K_{X / V}+E$ satisfies conditions (b) and (c) above. Let $\Gamma=\left(1 /\left(p^{e}-1\right)\right)\left(E+K_{X / V}+\Delta^{\prime}\right)$ for some $e \gg e_{0}$. Then consider $\Delta:=\Delta^{\prime}+\Gamma$. Note that

$$
\begin{aligned}
c\left(p^{e}-1\right)\left(K_{X / V}+\Delta\right) & =c\left(p^{e}-1\right)\left(K_{X / V}+\Delta^{\prime}+\Gamma\right) \\
& =c\left(p^{e}-1\right)\left(K_{X / V}+\Delta^{\prime}\right)+c\left(E+K_{X / V}+\Delta^{\prime}\right) \\
& =c p^{e}\left(K_{X / V}+\Delta^{\prime}\right)+c E,
\end{aligned}
$$

which is certainly Cartier. Hence $K_{X / V}+\Delta$ satisfies conditions (a)-(d) from Remark 2.11. On the other hand, since $e \gg 0$, we know that $\left(X_{s},\left.\Delta\right|_{X_{s}}\right)=\left(X_{s},\left.\Delta^{\prime}\right|_{X_{s}}+\left.\Gamma\right|_{X_{s}}\right)$ is still strongly $F$-regular, and so by Corollary 4.19 , there exists an open set $U$ such that $\left(X_{u},\left.\Delta\right|_{X_{u}}\right)$ is strongly $F$-regular for all $u \in U$. But $\Delta \geqslant \Delta^{\prime}$ and so the result follows for $\Delta^{\prime}$ as well.

Remark 4.21. In the case of a normal $X$ and in the non-relative case, we know that $\tau(X ; \Delta)=$ $\tau\left(\omega_{X}, K_{X}+\Delta\right)$. Furthermore, we then know that

$$
\operatorname{Tr}^{e}\left(F_{*}^{e} \tau\left(\omega_{X}, K_{X}+\Delta\right)\right)=\tau\left(\omega_{X}, \frac{1}{p^{e}}\left(K_{X}+\Delta\right)\right)=\tau\left(X, \frac{1}{p^{e}}\left(K_{X}+\Delta\right)-K_{X}\right) .
$$

Reversing this process gives us a nice means to compute $\tau(X, \Delta)$ when the index of $K_{X}+\Delta$ is divisible by $p$. It would be natural to try to prove a relative version of this, which may yield a suitable definition of relative test ideals for $K_{X}+\Delta$ of any index. We will not attempt this here. 


\section{Z. Patakfalvi, K. Schwede And W. Zhang}

\section{Relative test submodules, $F$-rationality, and $F$-injectivity}

Our goal in this (somewhat shorter) section is to explore relative test submodules and non- $F$ injective modules. Throughout this section, we assume that $f: X \rightarrow V$ is a Cohen-Macaulay morphism. This provides us with base change for relative canonical sheaves $\omega_{X / V}$ [Con00].

We first define relative non- $F$-injective modules $\sigma_{n}\left(X / V, \omega_{X / V}\right)$ and relative test submodules $\tau_{n}\left(X / V, \omega_{X / V}\right)$. Recall the trace map, Lemma 2.16:

$$
\Phi_{X / V, n}: \omega_{X / V}^{1 / p^{n}}=\omega_{X^{n} / V^{n}} \rightarrow \omega_{X_{V^{n} / V^{n}}} \cong \omega_{X / V} \otimes_{A} A^{1 / p^{n e}} .
$$

Here, the final isomorphism follows from [Con00, Theorem 3.6.1] since $f$ is a Cohen-Macaulay morphism. We will form $\sigma$ and $\tau$ relative to these maps, instead of relative to the map $\phi$ discussed previously. One key point to remember is that by Lemma 2.16, the map $\Phi_{X / V, n}$ is compatible with arbitrary base change (since in this section, $f$ is a Cohen-Macaulay morphism). We also observe that the composition of trace maps

$$
\omega_{X^{m} / V^{m}} \stackrel{\Phi_{X / V, m-n}^{1 / p^{m-n}}}{\longrightarrow} \omega_{X_{V}^{m} / V^{m}} \stackrel{\Phi_{X / V, n} \otimes_{A^{1 / p^{n}}} A^{1 / p^{m}}}{\longrightarrow} \omega_{X / V} \otimes_{A} A^{1 / p^{m}} \cong \omega_{X_{V^{m}} / V^{m}}
$$

can be identified with $\Phi_{X / V, m}$.

\subsection{The definition and basic properties of $\sigma_{n}\left(X / V, \omega_{X / V}\right)$}

For each integer $n>0$, define $\mathfrak{c}_{n}:=\operatorname{im}\left(\Phi_{X / V, n}\right) \subseteq \omega_{X_{V^{n} / V^{n}}} \cong \omega_{X / V} \otimes_{A} A^{1 / p^{n}}$. Furthermore, for each $m \geqslant n$, using the factorization in (5.1) it is easy to see that

$$
\mathfrak{c}_{m} \subseteq \operatorname{im}\left(\mathfrak{c}_{n} \otimes_{A^{1 / p^{n}}} A^{1 / p^{m}} \rightarrow \omega_{R_{A^{1 / p}}}\right),
$$

just as we observed in Section 3.

DeFinition 5.1. With notation as above, we define the $n$th relative non-F-injective submodule, denoted by $\sigma_{n}\left(X / V, \omega_{X / V}\right)$, to be $\mathfrak{c}_{n} \subseteq \omega_{X_{V^{n}} / V^{n}}$.

As before, we will prove a stabilization statement for $\mathfrak{c}_{n}$ and $\mathfrak{c}_{m}$, in particular that the containment (5.2) is an equality for all $m>n \gg 0$. By base changing with $k\left(V^{\infty}\right)$, the perfection of the residue field of the generic point of $V$, we can again find an integer $n_{0}>0$ and an open set $U \subseteq V$ such that

$$
\left.\mathfrak{c}_{n_{0}+1}\right|_{f^{-1} U}=\left.\operatorname{im}\left(\mathfrak{c}_{n_{0}} \otimes_{A^{1 / p^{n_{0}}}} A^{1 / p^{n_{0}+1}} \rightarrow \omega_{R}{ }_{A^{1 / p^{n_{0}+1}}}\right)\right|_{f^{-1} U}
$$

Again, observe that for $U$ inside the regular locus of $V$, we can identify the image above with $\left.\left(\mathfrak{c}_{n_{0}} \otimes_{A^{1 / p^{n}}{ }} A^{1 / p^{n_{0}+1}}\right)\right|_{U}$ by the flatness of $A^{1 / p^{m}}$ over $A^{1 / p^{n}}$. We then obtain the following result.

Proposition 5.2. Fix the notation as above. For every integer $n \geqslant n_{0}$, there exists a nonempty open subset $U_{n} \subseteq V$ of the base scheme $V$ satisfying the following condition. If one sets $X_{n}=f^{-1}\left(U_{n}\right)$, then for every $m \geqslant n$,

$$
\left.\sigma_{m}\left(X / V, \omega_{X / V}\right)\right|_{X_{n}}=\operatorname{im}\left(\sigma_{n}\left(X / V, \omega_{R / A}\right) \cdot \mathscr{O}_{X_{n}} \otimes_{A^{1 / p^{n}}} A^{1 / p^{m}} \rightarrow \omega_{R_{A^{1 / p^{m}}}}\right) .
$$

Furthermore, we may assume that $U_{n_{0}} \subseteq U_{n_{0}+1} \subseteq \cdots \subseteq U_{n} \subseteq U_{n+1} \subseteq \cdots$ form an ascending chain of open sets.

Proof. The proof is identical to that of Proposition 3.3 and so we omit it.

We now point out that relative non- $F$-injective modules behave well with respect to base change. Recall that if $g: T \rightarrow V$ is a map, then $q_{n}: X_{T^{n}} \rightarrow X_{V^{n}}$ is the induced map. 


\section{$F$-SINGULARITIES IN FAMILIES}

Proposition 5.3 (Base change for $\sigma_{n}\left(X / V, \omega_{X / V}\right)$ ). Suppose that $g: T \rightarrow V$ is a map from an excellent scheme with a dualizing complex; then using the notation of Section 2.2,

$$
\operatorname{im}\left(\left(q_{n}\right)^{*} \sigma_{n}\left(X / V, \omega_{X / V}\right) \rightarrow \omega_{X_{T^{n} / T^{n}}}\right)=\sigma_{n}\left(X_{T} / T, \omega_{X_{T} / T}\right) .
$$

Furthermore, if $U=U_{n}$ satisfies condition (5.3) from Proposition 5.2, then $W=g^{-1}(U) \subseteq T$ satisfies the same condition for $\sigma_{n}\left(X_{T} / T, \omega_{X_{T} / T}\right)$.

Proof. The first statement is an immediate consequence of base change relative to canonical sheaves and the trace map (Lemma 2.16). For the second, if $\Phi_{X / V, n} \otimes_{A^{1 / p^{n}}} A^{1 / p^{n+1}}$ and $\Phi_{X / V, n+1}$ have the same image in $\omega_{X_{V^{n}} / V^{n}}$, then it is easy to see that the base changed maps also have the same images.

Recall that for any $F$-finite scheme $Y$ with canonical module $\omega_{Y}$, the image $\sigma\left(\omega_{Y}\right)$ is equal to $\operatorname{im}\left(F_{*}^{e} \omega_{Y} \stackrel{\operatorname{Tr}^{e}}{\longrightarrow} \omega_{Y}\right)$ for any $e \gg 0$.

Corollary 5.4 (Restriction theorem for $\sigma_{n}\left(X / V, \omega_{X / V}\right)$ ). With notation as above, there exists an integer $N>0$ such that for every perfect point $s \in V$,

$$
\sigma\left(\omega_{X_{s}}\right)=\operatorname{im}\left(\sigma_{n}\left(X / V, \omega_{X / V}\right) \otimes_{A^{1 / p^{n e}}} k(s)^{1 / p^{n e}} \rightarrow \omega_{X_{s} / s}\right)
$$

for all $n>N$.

Proof. Taking $g: s \rightarrow V$ in Proposition 5.3, we obtain that the image in the statement of the proposition equals $\sigma_{n}\left(X_{s} / s, \omega_{X_{s} / s}\right)$. Furthermore, since $k(s)$ is perfect, we have containments $\cdots \supseteq \sigma_{n}\left(X_{s} / s, \omega_{X_{s} / s}\right) \supseteq \sigma_{n+1}\left(X_{s} / s, \omega_{X_{s} / s}\right) \supseteq \cdots$ with a descending intersection that coincides with $\sigma\left(\omega_{X_{s}}\right)$. Furthermore, by Proposition 5.3, over a dense open set $U \subseteq V$ and some $N>$ 0 , we have $\sigma_{n}\left(X_{s} / s, \omega_{X_{s} / s}\right)=\sigma_{n+1}\left(X_{s} / s, \omega_{X_{s} / s}\right)$ and hence $\sigma_{n}\left(X_{s} / s, \omega_{X_{s} / s}\right)=\sigma\left(\omega_{X_{s}}\right)$ by the construction of $\sigma\left(\omega_{X_{s}}\right)$ for all $n \geqslant N_{0}$. Let $V_{1}=V \backslash U$, base change with $V_{1}$, and obtain the result over a dense open subset $U_{1}$ of $V_{1}$ (for some $N_{1}$ ). By Noetherian induction, this process terminates.

\subsection{The definition and basic properties of $\tau_{n}\left(X / V, \omega_{X / V}\right)$}

The goal of this section is to develop the basics of a relative theory of test submodules. We fix the notation of the previous sections and additionally assume that $X$ is geometrically normal over $V$, which we now also assume is regular. We let $J=J_{X / V} \subseteq R$ be the Jacobian ideal sheaf of $X$ over $V$. We observe that the formation of $J_{X / V}$ commutes with base change in the following sense: for any $T \rightarrow V$, we have $J_{X / V} \cdot \mathscr{O}_{X_{T}}=J_{X_{T} / T}$. To see this, just note that $J_{X / V}$ can be defined as a Fitting ideal of $\Omega_{X / V}\left(\left[\right.\right.$ HS06, Discussion 4.4.7]) and that the formation of $\Omega_{X / V}$ ([Eis95, Proposition 16.4]) and Fitting ideals ([HS06, Discussion 4.4.7]) commutes with arbitrary base change. Note that $J$ is non-zero at any generic point of $X$ since $X$ is geometrically reduced. Furthermore, on every perfect fiber, the Jacobian ideal is contained in the (big) test ideal [Hoc07, Theorem on p. 213].

We have the images

$$
\begin{aligned}
& \mathfrak{d}_{1}:=\operatorname{im}\left(J \cdot \omega_{R_{A^{1 / p}} / A^{1 / p}} \rightarrow \omega_{R_{A^{1 / p}} / A^{1 / p}}\right)+\Phi_{X / V, 1}\left(\left(J \cdot \omega_{R}\right)^{1 / p}\right) \subseteq \omega_{R_{A^{1 / p}}}, \\
& \mathfrak{d}_{2}:=\sum_{i=0}^{2} \operatorname{im}\left(\left(\Phi_{X / V, i}\left(J \cdot \omega_{R / A}\right)^{1 / p^{i}}\right) \otimes_{A^{1 / p^{i}}} A^{1 / p^{2}} \rightarrow \omega_{R_{A^{1 / p^{2}}}}\right) \subseteq \omega_{R_{A^{1 / p^{2}}}},
\end{aligned}
$$




\section{Z. Patakfalvi, K. Schwede And W. Zhang}

$$
\mathfrak{d}_{n}:=\sum_{i=0}^{n} \operatorname{im}\left(\left(\Phi_{X / V, i}\left(J \cdot \omega_{R / A}\right)^{1 / p^{i}}\right) \otimes_{A^{1 / p^{i}}} A^{1 / p^{n}} \rightarrow \omega_{R_{A^{1 / p^{n}}}}\right) \subseteq \omega_{R_{A^{1 / p^{n}}}}
$$

Notice that $\mathfrak{d}_{1,2}:=\operatorname{im}\left(\mathfrak{d}_{1} \otimes_{A^{1 / p}} A^{1 / p^{2}} \rightarrow \omega_{R} A^{1 / p^{2}}\right) \subseteq \mathfrak{d}_{2}$ and more generally for $j>i$ that $\mathfrak{d}_{i, j}:=\operatorname{im}\left(\mathfrak{d}_{i} \otimes_{A^{1 / p^{i}}} A^{1 / p^{j}} \rightarrow \omega_{R_{A^{1 / p^{n}}}}\right) \subseteq \mathfrak{d}_{j}$

By the same argument as in Section 3, we know that there exists an open set $U \subseteq V$ with $W=f^{-1}(U)$ such that $\left.\operatorname{im}\left(\mathfrak{d}_{t} \otimes_{A^{1 / p^{t e}}} A^{1 / p^{(t+1) e}} \rightarrow \omega_{R} A_{A^{1 / p^{t+1}}}\right)\right|_{W}=\left.\mathfrak{d}_{t+1}\right|_{W}$.

LEMmA 5.5. With notation as above, $\left.\operatorname{im}\left(\mathfrak{d}_{n} \otimes_{A^{1 / p^{n e}}} A^{1 / p^{(n+1) e}} \rightarrow \omega_{R}{ }_{A^{1 / p}(n+1) e}\right)\right|_{W}=\left.\mathfrak{d}_{n+1}\right|_{W}$ for all $n>t$.

Proof. The proof is the same as the proof of Lemma 4.2 and so we omit it.

DEFINITION 5.6 (Relative test submodules). With notation as above (in particular, $\omega_{X / V}$ is still compatible with base change), we define the $n$th iterated relative test submodule to be $\mathfrak{d}_{n}$ and denote it by $\tau_{n}\left(X / V, \omega_{X / V}\right)$.

We now discuss base change for relative test ideals.

Proposition 5.7 (Base change for $\tau_{n}\left(X / V, \omega_{X / V}\right)$ ). Suppose that $g: T \rightarrow V$ is a map from a excellent scheme with a dualizing complex; then using the notation of Section 2.2,

$$
\operatorname{im}\left(\left(q_{n}\right)^{*} \tau_{n}\left(X / V, \omega_{X / V}\right) \rightarrow \omega_{X_{T^{n}} / T^{n}}\right)=\tau_{n}\left(X_{T} / T, \omega_{X / T}\right) .
$$

Furthermore, if $U=U_{n}$ satisfies condition from Lemma 5.5, then $Y=g^{-1}(U) \subseteq T$ satisfies the same condition for $\tau_{n}\left(X_{T} / T, \omega_{X_{T} / T}\right)$.

Proof. It is just as before since $\omega_{X / V}$ and $J$ are compatible with arbitrary base change.

Corollary 5.8 (Restriction theorem for $\tau_{n}\left(X / V, \omega_{X / V}\right)$ ). With notation as above, there exists an integer $N>0$ such that for every perfect point $s \in V$, we have

$$
\operatorname{im}\left(\tau_{n}\left(X / V, \omega_{X / V}\right) \otimes_{A^{1 / p^{n e}}} k(s)^{1 / p^{n e}} \rightarrow \omega_{X_{s} / s}\right)=\tau\left(\omega_{X_{s}}\right) .
$$

for all $n>N$.

Proof. Taking $g: s \rightarrow V$ in Proposition 5.7, we obtain that the image is equal to $\tau_{n}\left(X_{s} / s, \omega_{X_{s} / s}\right)$. Furthermore, since $k(s)$ is perfect, we can make identifications so as to have containments $\cdots \subseteq \tau_{n}\left(X_{s} / s, \omega_{X_{s} / s}\right) \subseteq \tau_{n+1}\left(X_{s} / s, \omega_{X_{s} / s}\right) \subseteq \cdots$ with an ascending union that coincides with $\tau\left(\omega_{X_{s}}\right)$. Furthermore, by Proposition 5.3, over a dense open set $U \subseteq V$ and some $N>0$, we have $\tau_{n}\left(X_{s} / s, \omega_{X_{s} / s}\right)=\tau_{n+1}\left(X_{s} / s, \omega_{X_{s} / s}\right)$ for all $n \geqslant N_{0}$ and hence $\tau_{n}\left(X_{s} / s, \omega_{X_{s} / s}\right)=$ $\tau\left(\omega_{X_{s}}\right)$ by the computation of (4.1). Let $V_{1}=V \backslash U$, base change with $V_{1}$, and obtain the result over a dense open subset $U_{1}$ of $V_{1}$ (for some $N_{1}$ ). By Noetherian induction, this process must terminate.

Remark 5.9 (Relative versus absolute $\tau(\omega)$ ). It would be natural to relate the relative $\sigma(\omega)$ and $\tau(\omega)$ to the absolute $\sigma(\omega)$ and $\tau(\omega)$. While the authors believe that this is possible along the lines of Section 3.1 or Section 4.1, we will not work out the details here. 


\section{$F$-SINGULARITIES IN FAMILIES}

\subsection{Applications to families of $\boldsymbol{F}$-injective and $\boldsymbol{F}$-rational singularities}

In this section, we obtain new proofs of results of Hashimoto [Has01], deformation of $F$-injectivity, and $F$-rationality in proper flat families. Note that Hashimoto proved the local version of these results (and in the $F$-rationality case, over a variety). However, the local results generalize to the non-local case via straightforward computations. We begin with a definition essentially first made by Hashimoto.

Definition 5.10 (cf. [Has01, Has10]). We say that $X / V$ (which is still assumed to be relatively Cohen-Macaulay) is relatively $F$-injective if for some $n>0$, we have $\sigma_{n}\left(X / V, \omega_{X / V}\right)=\omega_{X_{V^{n}} / V^{n}}$. Likewise, $X / V$ is relatively $F$-rational if for some $n>0$, we have $\tau_{n}\left(X / V, \omega_{X / V}\right)=\omega_{X_{V^{n}} / V^{n}}$.

Remark 5.11. If $V$ is the spectrum of a perfect field $k$, it is easy to see that $f: X \rightarrow V$ is relatively $F$-injective (respectively. relatively $F$-rational) if and only if $X$ is $F$-injective (respectively, $F$ rational) in the usual sense [ST12, Section 8] via an identification $k \cong k^{1 / p}$. Note that we are implicitly assuming that $X$ is Cohen-Macaulay in this case.

Lemma 5.12 (cf. [Has01, Proposition 5.5]). With notation as above, if $\sigma_{n}\left(X / V, \omega_{X / V}\right)=\omega_{X_{V^{n}} / V^{n}}$ for some $n>0$, then $\sigma_{m}\left(X / V, \omega_{X / V}\right)=\omega_{X_{V} / V^{m}}$ for all $m>0$ divisible by $n$. Furthermore, if $V$ is regular, then the result holds for all $m \geqslant n$. Additionally, if $\tau_{n}\left(X / V, \omega_{X / V}\right)=\omega_{X_{V^{n}} / V^{n}}$ for some $n>0$, then $\tau_{m}\left(X / V, \omega_{X / V}\right)=\omega_{X_{V} / V^{m}}$ for all $m \gg 0$.

Proof. We begin with $\sigma$. We notice that

$$
\sigma_{2 n}\left(X / V, \omega_{X / V}\right)=\operatorname{im}\left(\operatorname{im}\left(\omega_{X^{n} / V^{n}} \rightarrow \omega_{X_{V}^{n} / V^{n}}\right) \otimes_{A^{1 / p^{n}}} A^{1 / p^{2 n}} \rightarrow \omega_{X_{V^{2 n}} / V^{2 n}}\right)
$$

and our hypothesis $\sigma_{n}\left(X / V, \omega_{X / V}\right)=\omega_{X_{V^{n}} / V^{n}}$ implies that the inner map is surjective. But then, the outer map is surjective too by right exactness of the tensor product and so $\sigma_{2 n}\left(X / V, \omega_{X / V}\right)=$ $\omega_{X_{V^{2 n}} / V^{2 n}}$. The general case repeats this process and so follows similarly.

Now we assume that $V$ is regular for the second statement about $\sigma_{n}$. We fix an $n>0$ such

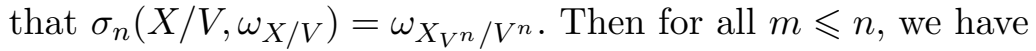

$$
\sigma_{m}\left(X / V, \omega_{X / V}\right) \otimes_{A^{1 / p^{m}}} A^{1 / p^{n}} \supseteq \sigma_{n}\left(X / V, \omega_{X / V}\right)=\omega_{X_{V^{n} / V^{n}}} \cong \omega_{X_{V^{m} / V^{m}}} \otimes_{A^{1 / p^{m}}} A^{1 / p^{n}}
$$

and so $\sigma_{m}\left(X / V, \omega_{X / V}\right) \otimes_{A^{1 / p^{m}}} A^{1 / p^{n}}=\omega_{X_{V} m / V^{m}} \otimes_{A^{1 / p^{m}}} A^{1 / p^{n}}$. On the other hand, if we know $\sigma_{m}\left(X / V, \omega_{X / V}\right) \subsetneq \omega_{X_{V^{m}} / V^{m}}$, then the previous equality is impossible since $A^{1 / p^{n}}$ is faithfully flat over $A$ [Kun69] (since $V$ is regular).

Handling $\tau$ is easy. Again use that $\omega_{X / V}$ is compatible with base change, since

$$
\begin{aligned}
\omega_{X_{V}^{m} / V^{m}} & =\operatorname{im}\left(\omega_{X / V} \otimes_{A^{1 / p^{n}}} A^{1 / p^{m}} \rightarrow \omega_{X_{V}^{m} / V^{m}}\right) \\
& =i m\left(\tau_{n}\left(X / V, \omega_{X / V}\right) \otimes_{A^{1 / p^{n}}} A^{1 / p^{m}} \rightarrow \omega_{X_{V}^{m} / V^{m}}\right) \\
& =\mathfrak{d}_{n, m} \subseteq \mathfrak{d}_{m}=\tau_{n}\left(X / V, \omega_{X / V} \subseteq \omega_{X_{V}^{m} / V^{m}} .\right.
\end{aligned}
$$

TheOREM 5.13 (Deformation of F-rationality and F-injectivity; cf. [Has01, Theorem 5.8, Remark 6.7]). Suppose that $f: X \rightarrow V$ is a proper, flat, finite-type, equidimensional, reduced Cohen-Macaulay morphism to an excellent integral scheme $V$ with a dualizing complex. Suppose that for some point $s \in V$, the fiber $X_{s} / s$ is relatively $F$-injective (respectively, $F$-rational). Then there exists an open neighborhood $U \subseteq V$ containing $s$ such that $X_{u} \rightarrow u$ is relatively $F$-injective (respectively, $F$-rational) for all $u \in U$.

Proof. We first show that $\sigma_{n}\left(\omega_{X / V}\right)=\omega_{X_{V} \text { ne } / V^{n e}}$ at each point of the fiber $X_{s}$. Indeed, let $z \in X$ be a point on $X_{s}$, and let $I$ denote the ideal sheaf of $X_{s}$. We observe that for some $n$, the natural 


\section{Z. Patakfalvi, K. Schwede And W. Zhang}

$\operatorname{map} \sigma_{n}\left(\omega_{X / V}\right) /\left(I \cdot \sigma_{n}\left(\omega_{X / V}\right)\right) \rightarrow \omega_{X / V} /\left(I \cdot \omega_{X / V}\right)=\omega_{X_{s} / V_{s}}$ is surjective. This is preserved after localizing at $z$, and so since $I \subseteq \mathfrak{m}_{z}$, we see that the generators of the stalk $\left(\sigma_{n}\left(\omega_{X / V}\right)\right)_{z}$ generate

$\left(\omega_{X / V}\right)_{z}$ by Nakayama's lemma. Hence $\left(\sigma_{n}\left(\omega_{X / V}\right)\right)_{z}=\left(\omega_{X / V}\right)_{z}$. Since this holds at every point $z \in X_{s} \subseteq X$, it holds in a neighborhood of $X_{s}$.

As before, let $Z$ denote the locus where $\sigma_{n}\left(\omega_{X / V}\right) \neq \omega_{X_{V} \text { ne } / V^{n e} \text {. This is closed and since } f \text { is }}$ proper, its image $f(Z)$ is closed too. But $f(Z)$ is then a closed set not containing $s$. The result follows for $F$-injectivity. The proof for $F$-rationality is the same.

\section{Global applications}

The purpose of this section is to develop a global theory of the previous sections for a projective family $f: X \rightarrow V$. In the last few years, there has been a new push to use the Frobenius morphism and the trace map to replace the Kodaira vanishing theorem. In this section, we extend some of these ideas to families. We study how the canonical linear subsystems $S^{0}\left(X_{s}, \sigma\left(X_{s}, \Delta_{s}\right) \otimes M_{s}\right) \subseteq$ $H^{0}\left(X_{s}, M_{s}\right)$, introduced in [Sch14], behave as we vary $s \in V$. Furthermore, as mentioned in the introduction, we also obtain some global generation and semi-positivity statements.

\subsection{Basic definitions}

We use the following setup throughout Section 6 .

Notation 6.1. In the situation of Notation 2.1, also assume that $f: X \rightarrow V$ is projective and that $V$ is regular (which implies that $F_{V}^{e}: V^{e} \rightarrow V$ is flat). Furthermore, fix a line bundle $M$ on $X$. Sometimes we also assume the following (in which case we write Notation $6.1^{*}$ ):

$(*)$ : There is an integer $N \geqslant 0$ such that for every integer $m \geqslant N$,

$$
\sigma_{m}(X / V, \phi)=\sigma_{N}(X / V, \phi) \otimes_{A^{1 / p^{N e}}} A^{1 / p^{m e}} .
$$

In this situation, we denote $\sigma_{N}(X / V, \phi)$ by $\sigma_{N}$. We notice that this condition (*) always holds over a dense open set of the base by Proposition 3.3.

Remark 6.2. Note that since $X$ (respectively, $V$ ) is topologically isomorphic to $X_{V^{m e}}^{n e}$ (respectively, $V^{m e}$ ) for every $m \geqslant n$, the push-forward $f_{*}$ can be identified with $g_{*}$, where $g$ is any of the induced morphisms $X_{V^{m e}}^{n e} \rightarrow V^{m e}$. Hence, we use only $f_{*}$ for all purposes, even when $g_{*}$ for one of the above maps $g: X_{V^{m e}}^{n e} \rightarrow V^{m e}$ would be more natural. The downside of this notation is that it does not show if a sheaf has an $A^{1 / p^{m e}}$-structure and consequently its pushforward by

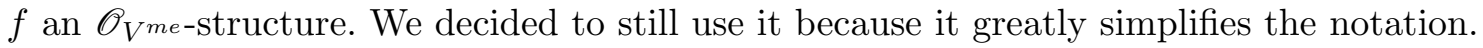

Summarizing: When reading the following arguments, it is important to trace through the space $X_{V m e}^{n e}$ on which the adequate sheaves live. Then $f_{*}$ of these sheaves will live on $V^{m e}$.

Notation 6.3. When dealing with sheaves $G$ on $V^{\text {ne }}$, we will frequently pull them back to $V^{m e}$ for $m \geqslant n$. When doing this, instead of writing $\mathscr{O}_{V^{m e}} \otimes_{\mathscr{O}_{V} \text { ne }} G$ or $\mathscr{O}_{V}^{1 / p^{m e}} \otimes_{\mathscr{O}_{V}^{1 / p^{n e}}} G$, we will write simply $V^{m e} \times_{V^{n e}} G$ or $G_{V^{m e}}$. We trust that this abuse of notation will cause no confusion, as it helps compactify the notation substantially.

Our approach to understanding how the canonical linear systems of [Sch14] behave in families is to define a relative version of them and then show certain base-change properties. These objects will be relative versions of $\sigma$ for global sections, and they play the same role for $\sigma_{n}$ that $T^{0}$ and $S^{0}$ play for $\tau$ and $\sigma$ in [BST15] and [Sch14], respectively. 


\section{$F$-SINGULARITIES IN FAMILIES}

DeFinition 6.4. In the situation of Notation 6.1, define

$$
S_{\varphi^{n}}^{0} f_{*}(M):=\operatorname{im}\left(f_{*}\left(\left(L^{\frac{p^{n e}-1}{p^{e}-1}}\right)^{1 / p^{n e}} \otimes_{R} M\right) \stackrel{f_{*}\left(\varphi^{n} \otimes_{R} \operatorname{id}_{M}\right)}{\longrightarrow} f_{*}\left(A^{1 / p^{n e}} \otimes_{A} M\right)\right) .
$$

Note that $S_{\varphi^{n}}^{0} f_{*}(M)$ is a sheaf on $V^{n e}$; it is a subsheaf of $\left(f_{*}(M)\right)_{V^{n e}}$ by flat base change. In case $(X, \Delta)$ is a pair, we define $S_{\Delta, n e}^{0} f_{*}(M):=S_{\varphi_{\Delta}^{n}}^{0} f_{*}(M)$ (assuming that $\left(p^{n e}-1\right)\left(K_{X}+\Delta\right)$ is Cartier and $\phi$ is the corresponding map). If $\Delta=0$, then we write $S_{n e}^{0} f_{*}(M)$ for $S_{\Delta, n e}^{0} f_{*}(M)$.

Since the image of $\varphi^{n} \otimes_{R} \operatorname{id}_{M}$ in the above definition is $\sigma_{n}(X / V, \phi) \otimes_{R} M$, the following proposition is immediate.

Proposition 6.5. In the situation of Notation 6.1, we have $S_{\varphi^{n}}^{0} f_{*}(M) \subseteq f_{*}\left(\sigma_{n}(X / V, \phi) \otimes_{R} M\right)$.

Our first goal is to show that the images in Definition 6.4 descend (up to appropriate base change by the Frobenius morphism). Compare with the containments $\mathfrak{a}_{i, n} \supseteq \mathfrak{a}_{i+1, n} \supseteq \cdots \supseteq \mathfrak{a}_{n, n}$ of Section 3.

Proposition 6.6. For all integers $m \geqslant n \geqslant 0$,

$$
V^{m e} \times_{V^{n e}} S_{\varphi^{n}}^{0} f_{*}(M) \supseteq S_{\varphi^{m}}^{0} f_{*}(M)
$$

as subsheaves of $V^{m e} \times_{V} f_{*}(M)$.

Remark 6.7. To be precise, the left- and right-hand sides of (6.1) are subsheaves of

$$
V^{m e} \times_{V^{n e}} f_{*}\left(A^{1 / p^{n e}} \otimes_{A} M\right) \text { and } f_{*}\left(A^{1 / p^{m e}} \otimes_{A} M\right),
$$

respectively. However, both $V^{m e} \times_{V^{n e}} f_{*}\left(A^{1 / p^{n e}} \otimes_{A} M\right)$ and $f_{*}\left(A^{1 / p^{m e}} \otimes_{A} M\right)$ are canonically isomorphic to $V^{m e} \times_{V} f_{*}(M)$ via flat base change (since $V$ is regular).

Proof of Proposition 6.6. The following commutative diagram shows that $f_{*}\left(\varphi^{m} \otimes_{R} \mathrm{id} M\right)$ factors through $V^{m e} \times_{V^{n e}} f_{*}\left(\varphi^{n} \otimes_{R} \operatorname{id}_{M}\right)$ :

$$
\begin{aligned}
& V^{m e} \times_{V^{n e}} f_{*}\left(\left(L^{\frac{p^{n e}-1}{p^{e}-1}}\right)^{1 / p^{n e}} \otimes_{R} M\right) \stackrel{V^{m e} \times_{V^{n e}} f_{*}\left(\varphi^{n} \otimes_{R} \operatorname{id}_{M}\right)}{\longrightarrow} V^{m e} \times_{V^{n e}} f_{*}\left(A^{1 / p^{n e}} \otimes_{A} M\right) \\
& \begin{array}{l}
\text { flat base change [Har77, } \\
\| 2 \begin{array}{l}
\text { Proposition III.9.3] of } f_{*} \\
\text { by } V^{m e} \longrightarrow V^{n e}
\end{array}
\end{array} \quad \| l_{\text {change }}^{\text {flat base }} \\
& f_{*}\left(A^{1 / p^{m e}} \otimes_{A^{1 / p^{n e}}}\left(L^{\frac{p^{n e}-1}{p^{e}-1}}\right)^{1 / p^{n e}} \otimes_{R} M\right) \stackrel{f_{*}\left(A^{1 / p^{m e}} \otimes_{A^{1 / p^{n e}}}\left(\varphi^{n} \otimes_{R^{1}} \mathrm{id}_{M}\right)\right)}{\longrightarrow} f_{*}\left(A^{1 / p^{m e}} \otimes_{A} M\right) \\
& \mathbb{1 2} \\
& f_{*}\left(\left(R_{A^{1 / p}(m-n) e} \otimes_{R} L^{\frac{p^{n e}-1}{p^{e}-1}}\right)^{1 / p^{n e}} \otimes_{R} M\right) \longrightarrow f_{*}\left(A^{1 / p^{m e}} \otimes_{A} M\right) \\
& \uparrow f_{*}\left(\left(\phi^{m-n} \otimes_{R} \mathrm{id}_{L} \frac{p^{n e}-1}{p^{e}-1}\right)^{1 / p^{n e}} \otimes_{R} \mathrm{id}_{M}\right) \\
& f_{*}\left(\left(\left(L^{\frac{p^{(m-n) e}-1}{p^{e}-1}}\right)^{1 / p^{(m-n) e}} \otimes_{R} L^{\frac{p^{n e}-1}{p^{e}-1}}\right)^{1 / p^{n e}} \otimes_{R} M\right) \\
& \| 2 \text { projection formula } \\
& f_{*}\left(\left(L^{\frac{p^{m e}-1}{p^{e}-1}}\right)^{1 / p^{m e}} \otimes_{R} M\right)
\end{aligned}
$$




\section{Z. Patakfalvi, K. Schwede And W. Zhang}

Hence, the statement of the proposition holds by the following computation:

$$
\begin{aligned}
S_{\phi^{m}}^{0} f_{*}(M) & =\operatorname{im}\left(f_{*}\left(\varphi^{m} \otimes_{R} \operatorname{id}_{M}\right)\right) \\
& \subseteq \operatorname{im}\left(V^{m e} \times_{V^{n e}} f_{*}\left(\varphi^{n} \otimes_{R} \operatorname{id}_{M}\right)\right) \\
& =\underbrace{V^{m e} \times_{V^{n e}} \operatorname{im}\left(f_{*}\left(\varphi^{n} \otimes_{R} \operatorname{id}_{M}\right)\right)}_{V^{n e} \longrightarrow V^{m e} \text { is flat }} \\
& =V^{m e} \times_{V^{n e}} S_{\phi^{n}}^{0} f_{*}(M) .
\end{aligned}
$$

\subsection{Auxiliary definition and stabilization}

We would now like to obtain a global result similar to Proposition 3.3. In particular, we would like the containments of Proposition 6.6 to be equalities over a dense open subset of the base $V$. There is a complicating factor, however: while we can still find an open set $U$ of $V$ such that

$$
\left.\left(V^{(n+1) e} \times_{V^{n e}} S_{\varphi^{n}}^{0} f_{*}(M)\right)\right|_{U}=\left.\left(S_{\varphi^{n+1}}^{0} f_{*}(M)\right)\right|_{U},
$$

we do not see how to use this to show that we have the $n+1$ to $n+2$ equality without additional assumptions. The issue is that in the proof of Proposition 3.3, twisting by line bundles is exact. For $S_{\phi^{n}}^{0}$, however, we also push forward. Therefore, in order to obtain our stabilization over a dense open set of the base, we need additional positivity assumptions on $M$ and $L$.

Furthermore, we need an auxiliary version of $S_{\phi}^{0}$ which involves the $\sigma_{N}:=\sigma_{N}(X / V, \phi)$ from Notation $6.1^{*}$. To do this, first observe that since $f$ is flat, the tensor product $L^{\frac{p^{m e}-1}{p^{e}-1}} \otimes_{R} \sigma_{N}$ is naturally identified with a subsheaf of $L^{\frac{p^{m e}-1}{p^{e}-1}} \otimes_{R} R_{A^{1 / p^{N e}}} \cong L^{\frac{p^{m e}-1}{p^{e}-1}} \otimes_{A} A^{1 / p^{N e}}$. In order to motivate this auxiliary definition, we make the following observation.

Lemma 6.8. In the situation of Notation $6.1^{*}$, the image of the natural map

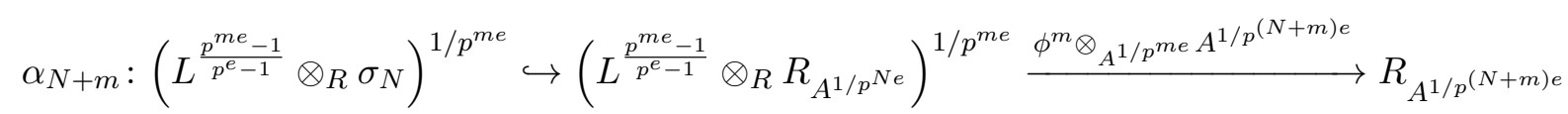

is equal to $\sigma_{N} \otimes A^{1 / p^{N e}} A^{1 / p^{(N+m) e}} \cong \sigma_{m+N}(X / V, \phi)$.

Proof. The first term is the image of the $p^{m e}$ th root of $L^{\frac{p^{m e}}{p^{e}-1}} \otimes_{R} \phi^{N}$. Therefore, the image of the composition also equals $\sigma_{m+N}(X / V, \phi)$, by our construction of $\phi^{j}$ in Section 2.1.

This stabilization suggests that it is reasonable to make the following definition.

DeFinition 6.9. In the situation of Notation $6.1^{*}$,

$$
S_{\varphi^{n}, \sigma_{N}}^{0} f_{*}(M):=\operatorname{im}\left(f_{*}\left(\left(L^{\frac{p^{m e}-1}{p^{e}-1}} \otimes_{R} \sigma_{N}\right)^{1 / p^{m e}} \otimes_{R} M\right) \stackrel{f_{*}\left(\alpha_{N+m} \otimes_{R} M\right)}{\longrightarrow} f_{*}\left(A^{1 / p^{(n+N) e}} \otimes_{A} M\right)\right),
$$

where $\alpha_{N+m}$ is as in Lemma 6.8.

Consider the following proposition relating the various $S^{0} f_{*}$-objects described so far.

Proposition 6.10. In the situation of Notation $6.1^{*}$, for every integer $m \geqslant 0$,

$$
V^{(N+m) e} \times_{V^{m e}} S_{\varphi^{m}}^{0} f_{*}(M) \supseteq S_{\varphi^{m}, \sigma_{N}}^{0} f_{*}(M) \supseteq S_{\varphi^{m+N}}^{0} f_{*}(M) .
$$

(Here, all sheaves are regarded as subsheaves of $V^{(N+m) e} \times_{V} f_{*}(M)$ via flat base change.)

Proof. This follows directly from the definitions. The first containment is trivial, and the second follows from a factorization similar to the one from Proposition 6.6. 


\section{$F$-SINGULARITIES IN FAMILIES}

Proposition 6.11. In the situation of Notation $6.1^{*}$,

(a) for all integers $m \geqslant n \geqslant 0$,

$$
V^{(N+m) e} \times_{V^{(N+n) e}} S_{\varphi^{n}, \sigma_{N}}^{0} f_{*}(M) \supseteq S_{\varphi^{m}, \sigma_{N}}^{0} f_{*}(M),
$$

as subsheaves of $V^{(N+m) e} \times_{V} f_{*}(M)$;

(b) if, furthermore, $L \otimes M^{p^{e}-1}$ is $f$-ample, then there is an integer $n>0$ such that for every integer $m \geqslant n$, the above inclusion is an equality;

(c) if, furthermore, $M=Q^{l} \otimes P$, where $Q$ is $f$-ample, then there is an integer $l_{0}>0$ such that for every integer $l \geqslant l_{0}, m \geqslant n \geqslant 0$, and nef line bundle $P$, the above inclusion is an equality.

Proof. Define the coherent sheaf $B_{\phi}$ on $X_{V^{(N+1) e}}$ as the kernel of the top horizontal map in the following commutative diagram:

$$
\begin{aligned}
& B_{\phi} \longleftrightarrow\left(L \otimes_{R} \sigma_{N}\right)^{1 / p^{e}} \longrightarrow \phi \otimes A^{1 / p^{(N+1) e}} \otimes_{A^{1 / p^{N e}}} \sigma_{N} \\
& \left(\operatorname{id}_{L} \otimes_{R} \phi^{N}\right)^{1 / p^{e}} \uparrow \quad \uparrow^{N+1} \\
& \left(L \otimes\left(L^{\frac{p^{N e}-1}{p^{e}-1}}\right)^{1 / p^{N e}}\right)^{1 / p^{e}} \cong\left(L^{\frac{p^{(N+1) e}-1}{p^{e}-1}}\right)^{1 / p^{(N+1) e}} .
\end{aligned}
$$

Note that here, we used that the image of $\phi^{N+1}$ is $A^{1 / p^{(N+1) e}} \otimes_{A^{1 / p^{N e}}} \sigma_{N}$ by the assumptions made in Notation $6.1^{*}$. Also, the horizontal arrow is surjective by either Lemma 6.8 or from the diagram (which is how we proved Lemma 6.8). Then one can apply $f_{*}\left(\left(L^{\frac{p^{m e}-1}{p^{e}-1}} \otimes_{R}-\right)^{1 / p^{m e}} \otimes_{R} M\right)$ to the top row of (6.2), which is shown in the following commutative diagram. We have also included important isomorphisms to the different terms of the exact sequence

$$
\begin{gathered}
f_{*}\left(\left(L^{\frac{p^{m e}-1}{p^{e}-1}} \otimes_{R} B_{\phi}\right)^{1 / p^{m e}} \otimes_{R} M\right) \\
f_{*}\left(\left(L^{\frac{p^{m e}-1}{p^{e}-1}} \otimes_{R}\left(L \otimes \sigma_{N}\right)^{1 / p^{e}}\right)^{1 / p^{m e}} \otimes_{R} M\right) \cong f_{*}\left(\left(L^{\frac{p^{(m+1) e}-1}{p^{e}-1}} \otimes_{R} \sigma_{N}\right)^{1 / p^{(m+1) e}} \otimes_{R} M\right) \\
f_{*}\left(\left(L^{\frac{p^{m e}-1}{p^{e}-1}} \otimes_{R}\left(\sigma_{N} \otimes_{A^{1 / p^{N e}}} A^{1 / p^{(N+1) e}}\right)\right)^{1 / p^{m e}} \otimes_{R} M\right) \\
R_{*}^{1} f_{*}\left(\left(L^{\frac{p^{m e}-1}{p^{e}-1}} \otimes_{R} B_{\phi}\right)^{1 / p^{m e}} \otimes_{R} M\right) \\
\left.f_{*}\left(\left(L^{\frac{p^{m e}-1}{p^{e}-1}} \otimes_{R} \sigma_{N}\right)^{1 / p^{m e}} \otimes_{A^{1 / p^{(N+m) e}}} A^{1 / p^{(N+m+1) e}}\right) \otimes_{R} M\right) \\
\left.\mathbb{I R}_{\text {by flat base change }}^{(N+m+1) e} \times_{V^{(N+m) e} f_{*}\left(\left(L^{\frac{p^{m e}-1}{p^{e}-1}} \otimes_{R} \sigma_{N}\right)^{1 / p^{m e}}\right.} \otimes_{R} M\right) \\
V^{(N+m+1) e} \times_{V^{(N+m) e}} S_{\phi^{m}, \sigma_{N}}^{0} f_{*}(M)<
\end{gathered} \mid
$$




\section{Z. Patakfalvi, K. Schwede and W. Zhang}

Both $V^{(N+m+1) e} \times_{V^{(N+m) e}} S_{\phi^{m}, \sigma_{N}}^{0} f_{*}(M)$ and $S_{\phi^{m+1}, \sigma_{N}}^{0} f_{*}(M)$ can be regarded as subsheaves of $V^{(N+m+1) e} \times_{V} f_{*}(M)$ via flat base change. Furthermore, the bottom horizontal arrow becomes a map of subsheaves, that is, an injection, this way. This shows point (a).

To prove point (b), we are supposed to prove that whenever $L \otimes M^{p^{e}-1}$ is $f$-ample, this arrow is also surjective for $m \gg 0$. This would follow if the third vertical arrow (from above), labeled $\nu$, is surjective. Therefore, it is sufficient to show that for $m \gg 0$,

$$
\begin{aligned}
0 & =R^{1} f_{*}\left(\left(L^{\frac{p^{m e}-1}{p^{e}-1}} \otimes_{R} B_{\phi}\right)^{1 / p^{m e}} \otimes_{R} M\right)=R^{1} f_{*}\left(\left(L^{\frac{p^{m e}-1}{p^{e}-1}} \otimes_{R} B_{\phi} \otimes_{R} M^{p^{m e}}\right)^{1 / p^{m e}}\right) \\
& =R^{1} f_{*}\left(\left(\left(L \otimes M^{p^{e}-1}\right)^{\frac{p^{m e}-1}{p^{e}-1}} \otimes_{R} B_{\phi} \otimes_{R} M\right)^{1 / p^{m e}}\right) .
\end{aligned}
$$

Note that since applying $\left(\_\right)^{1 / p^{m e}}$ does not change the sheaf of abelian groups structure, this is equivalent to showing

$$
\begin{aligned}
0 & =R^{1} f_{*}\left(\left(L \otimes M^{p^{e}-1}\right)^{\frac{p^{m e}-1}{p^{e}-1}} \otimes_{R} B_{\phi} \otimes_{R} M\right) \\
& =R^{1} f_{*}\left(\left(L \otimes M^{p^{e}-1} \otimes_{A} A^{1 / p^{N+1}}\right)^{\frac{p^{m e}-1}{p^{e}-1}} \otimes_{A^{1 / p^{(N+1) e}}}\left(B_{\phi} \otimes_{R} M\right)\right) .
\end{aligned}
$$

Furthermore, $L \otimes M^{p^{e}-1} \otimes_{A} A^{1 / p^{N+1}}$ is a relatively ample line bundle by the assumption of point (b) and $B_{\phi} \otimes_{R} M$ is a coherent sheaf on $X_{V^{(N+1) e}}$. Therefore, the relative Serre vanishing theorem concludes our proof.

Point (c) follows immediately from the above argument. Indeed, if $M=Q^{l} \otimes P$, then by the relative Fujita vanishing theorem [Kee03, Theorem 1.5], there is an integer $l_{0}>0$ such that the above vanishing holds for every $m \geqslant 0, l \geqslant l_{0}$, and nef line bundle $P$.

Corollary 6.12. In the situation of Notation $6.1^{*}$, if $L \otimes M^{p^{e}-1}$ is $f$-ample, then there is an integer $n>0$ such that for every integer $m \geqslant n$,

$$
V^{m e} \times_{V^{n e}} S_{\varphi^{n}}^{0} f_{*}(M)=S_{\varphi^{m}}^{0} f_{*}(M) \quad\left(=S_{\varphi^{m-N}, \sigma_{N}}^{0} f_{*}(M)\right)
$$

as subsheaves of $V^{m e} \times_{V} f_{*}(M)$. Furthermore, in the situation of point (c) of Proposition 6.11, where $M=Q^{l} \otimes P=(\text { ample })^{l} \otimes($ nef $)$ with $l \gg 0$, we can pick $n=N$.

Proof. By Proposition 6.10,

$$
V^{(m+2 N) e} \times_{V^{(m+N) e}} S_{\varphi^{m}, \sigma_{N}}^{0} f_{*}(M) \supseteq V^{(m+2 N) e} \times_{V^{(m+N) e}} S_{\varphi^{m+N}}^{0} f_{*}(M) \supseteq S_{\varphi^{m+N}, \sigma_{N}}^{0} f_{*}(M) .
$$

Furthermore, by Proposition 6.11(b), we know that the statement holds when $S_{\varphi^{m}}^{0} f_{*}(M)$ is replaced by $S_{\varphi^{m}, \sigma_{N}}^{0} f_{*}(M)$. Hence, the inclusion of the right end of (6.3) in the left end is an equality for $m \gg 0$. However, then all inclusions in (6.3) are equalities. In particular, from the first equality of $(6.3)$, using that $V^{(m+2 N) e} \rightarrow V^{(m+N) e}$ is faithfully flat, we obtain that $S_{\varphi^{m}, \sigma_{N}}^{0} f_{*}(M)=S_{\varphi^{m+N}} f_{*}(M)$ for $m \gg 0$. Using Proposition 6.11(b) once more concludes our proof of the main statement. The final statement is similar.

We can now obtain our promised analog of Proposition 3.3.

TheOREM 6.13. In the situation of Notation 6.1, if $L \otimes M^{p^{e}-1}$ is ample, there exist an integer $n_{0}$ and a dense open set $U \subseteq V$ such that for all $m \geqslant n \geqslant n_{0}$, we have

$$
\left.\left(V^{m e} \times_{V^{n e}} S_{\varphi^{n}}^{0} f_{*}(M)\right)\right|_{U}=\left.\left(S_{\varphi^{m}}^{0} f_{*}(M)\right)\right|_{U} .
$$




\section{$F$-SINGULARITIES IN FAMILIES}

Proof. By shrinking $V$ and applying Proposition 3.3, we can reduce to the case of Notation $6.1^{*}$. The result follows directly from Corollary 6.12.

COROLlary 6.14. In the situation of Notation 6.1, there is a natural inclusion

$$
S_{\phi^{n}}^{0} f_{*}(M) \subseteq f_{*}\left(\sigma_{n}(X / V, \phi) \otimes_{R} M\right)
$$

as subsheaves of $V^{n e} \times_{V} f_{*}(M)$.

Further, in the situation of Notation $6.1^{*}$, if $M=Q^{l} \otimes P$, where $Q$ is $f$-ample, then there is an integer $l_{0}>0$ such that for every integer $l \geqslant l_{0}, n \geqslant N$, and $f$-nef line bundle $P$, the above inclusion is an equality.

Proof. Consider the surjection

$$
\xi:\left(L^{\frac{p^{n e}-1}{p^{e}-1}}\right)^{1 / p^{n e}} \longrightarrow \phi_{n} \longrightarrow \sigma_{n}(X / V, \phi) .
$$

Then $S_{\phi^{n}}^{0} f_{*}(M)=\operatorname{im} f_{*}\left(\xi \otimes_{R} \operatorname{id}_{M}\right)$, which is a subsheaf of $f_{*}\left(\sigma_{n}(X / V, \phi) \otimes_{R} M\right)$.

We prove the addendum by induction on $n$. If $n=N$,

$$
S_{\phi^{N}}^{0} f_{*}(M)=\underbrace{S_{\phi^{0}, \sigma_{N}}^{0} f_{*}(M)}_{\text {by Corollary 6.12 }}=\underbrace{f_{*}\left(\sigma_{N} \otimes_{R} M\right)}_{\text {by Definition 6.9 }} .
$$

Let us then assume $n>N$. By flat base change and the assumption of Notation $6.1^{*}$,

$$
f_{*}\left(\sigma_{n}(X / V) \otimes_{R} M\right)=f_{*}\left(\sigma_{N}(X / V) \otimes_{R} M\right) \times_{V^{N e}} V^{n e} .
$$

Hence, using the induction hypothesis, it is enough to see that for every $n \geqslant N$,

$$
S_{\phi^{n}}^{0} f_{*}(M)=S_{\phi^{n-1}}^{0} f_{*}(M) .
$$

However, this follows from point (c) of Proposition 6.11 and Corollary 6.12.

\subsection{Base change}

We now prove base change for $S_{\phi^{n}}^{0} f_{*}$.

Notation 6.15. In the situation of Notation 6.1, choose

- a regular, integral, excellent scheme $T$ with a dualizing complex,

$\circ$ a morphism $T \rightarrow V$.

We indicate base change via this morphism by $T$ in subscript. Define the following sheaves on $X_{T}$ :

(a) $B:=\left(f_{T}\right)^{-1} \mathscr{O}_{T}$,

(b) $Q:=\mathscr{O}_{X_{T}}$.

Denote by $\left(p_{1}\right)^{1 / p^{i}}$ the natural projection morphism $X^{i} \times_{V^{i}} T^{i} \rightarrow X^{i}$.

Proposition 6.16. In the situation of Notation 6.15, if $L \otimes M^{p^{e}-1}$ is $f$-ample, then for $n \gg 0$, the natural base change morphism

$$
f_{*}\left(A^{1 / p^{n e}} \otimes_{A} M\right) \times_{V^{n e}} T^{n e} \rightarrow\left(f_{T}\right)_{*}\left(B^{1 / p^{n e}} \otimes_{B} M_{T}\right)
$$

induces a surjective morphism on subsheaves

$$
S_{\phi^{n}}^{0} f_{*}(M) \times_{V^{n e}} T^{n e} \rightarrow S_{\phi_{T}^{n}}^{0}\left(f_{T}\right)_{*}\left(M_{T}\right) .
$$

Furthermore, the following hold: 


\section{Z. Patakfalvi, K. Schwede And W. Zhang}

(a) The lower bound on the $n$ for which the above statement holds depends only on $f$ and $M$. In particular, it is independent of $T$.

(b) If $M=Q^{l} \otimes P$ for some $f$-ample line bundle $Q$, nef line bundle $P$, and integer $l>0$, then there is a uniform lower bound on $n$ independent of $l$ and $P$.

(c) Even if $L \otimes M^{p^{e}-1}$ is not assumed to be $f$-ample, (6.4) is an isomorphism if $T \rightarrow V$ is flat.

(d) There is a dense open set $U \subseteq V$ such that if the image of $T \rightarrow V$ is contained in $U$, then (6.4) is an isomorphism.

Proof of Proposition 6.16. First, note that

$$
\begin{aligned}
\left(\left(p_{1}\right)^{1 / p^{n e}}\right)^{*}\left(\left(L^{\frac{p^{n e}-1}{p^{e}-1}} \otimes_{R} M^{p^{n e}}\right)^{1 / p^{n e}}\right) & \cong\left(\left(p_{1}\right)^{1 / p^{n e}}\right)^{*}\left(\left(L^{\frac{p^{n e}-1}{p^{e}-1}}\right)^{1 / p^{n e}} \otimes_{R} M\right) \\
& \cong\left(L_{T}^{\frac{p^{n e}-1}{p^{e}-1}}\right)^{1 / p^{n e}} \otimes_{Q} M_{T} .
\end{aligned}
$$

Hence, there is a natural base-change morphism below in (6.5). We will show that it is an isomorphism for $n \gg 0$. Furthermore, this $n$ can be chosen independently of $T$.

$$
\left(f_{*}\left(\left(L^{\frac{p^{n e}-1}{p^{e}-1}}\right)^{1 / p^{n e}} \otimes_{R} M\right)\right) \times_{V^{n e}} T^{n e} \rightarrow\left(f_{T}\right)_{*}\left(\left(L_{T}^{\frac{p^{n e}-1}{p^{e}-1}}\right)^{1 / p^{n e}} \otimes_{Q} M_{T}\right) .
$$

Indeed, by cohomology and base change [Sta17, Lemma 25.20.1], it is enough to show that for every integer $i>0$,

$$
R^{i} f_{*}\left(\left(L^{\frac{p^{n e}-1}{p^{e}-1}}\right)^{1 / p^{n e}} \otimes_{R} M\right)=0 .
$$

Since $V^{n e} \rightarrow V$ is affine, this is equivalent to showing that for $i>0$,

$$
0=R^{i}\left(f_{V^{n e}}\right)_{*}\left(\left(L^{\frac{p^{n e}-1}{p^{e}-1}}\right)^{1 / p^{n e}} \otimes_{R} M\right)=R^{i}\left(f_{V^{n e}}\right)_{*}\left(\left(L^{\frac{p^{n e}-1}{p^{e}-1}} \otimes_{R} M^{p^{n e}}\right)^{1 / p^{n e}}\right)
$$

which is further equivalent to showing that for $i>0$,

$$
0=R^{i} f_{*}\left(L^{\frac{p^{n e}-1}{p^{e}-1}} \otimes_{R} M^{p^{n e}}\right) .
$$

However, the last vanishing holds for $n \gg 0$ by the relative Serre vanishing theorem, independently of $T$. Hence, the base-change homomorphism of (6.5) is indeed an isomorphism for $n \gg 0$, which can be chosen independently of $T$. In particular, for $n \gg 0$, there is a commutative base-change diagram as follows, which implies the statement of the proposition together with addendum (a):

$$
\begin{gathered}
f_{*}\left(\left(L^{\frac{p^{n e}-1}{p^{e}-1}}\right)^{1 / p^{n e}} \otimes_{R} M\right) \times_{V^{n e}} T^{n e} \longrightarrow f_{*}\left(A^{1 / p^{n e}} \otimes_{A} M\right) \times_{V^{n e}} T^{n e} \\
\mathbb{R} \\
\left(f_{T}\right)_{*}\left(\left(L_{T}^{\frac{p^{n e}-1}{p^{e}-1}}\right)^{1 / p^{n e}} \otimes_{Q} M_{T}\right) \longrightarrow \underset{\downarrow}{\longrightarrow}\left(f_{T}\right)_{*}\left(B^{1 / p^{n e}} \otimes_{B} M_{T}\right) .
\end{gathered}
$$

For addendum (c), note that if $T \rightarrow V$ is flat, then both vertical morphisms in (6.7) are isomorphisms even if $L \otimes M^{p^{e}-1}$ is not assumed to be $f$-ample. Furthermore, images via flat pullbacks are pullbacks of images, which concludes the proof of addendum (c).

For addendum (b), note that if $M=Q^{l} \otimes P$ for an $f$-ample line bundle $Q$ and a nef line 


\section{$F$-SINGULARITIES IN FAMILIES}

bundle $P$, then (6.6) holds for every $l>0$ uniformly, by the relative Fujita vanishing theorem [Kee03].

For addendum (d), note that by [Har77, Theorem 12.8 and Corollary 12.9], there is a dense open set $U \subseteq V$ (that is, the open set where $h^{0}\left(X_{s}, M_{s}\right)$ is constant) such that if $T$ maps into $U$, then the right vertical arrow in (6.7) is an isomorphism. In particular, the homomorphism (6.4) is then injective, because it is a homomorphism between subsheaves of the two sheaves involved in the above vertical map. Therefore, by the already proven surjectivity, (6.4) is an isomorphism. This concludes addendum $(\mathrm{d})$.

Remark 6.17. In particular, if in Notation $6.15, T=s$ is a perfect point of $V$ (that is, $T=$ $\operatorname{Spec}(K)$ for some perfect field $K)$, then $S_{\phi_{T}^{n}}^{0}\left(f_{T}\right)_{*}\left(M_{T}\right)$ can be interpreted as follows. Since the natural map $K \rightarrow(K)^{1 / p^{i}}$ is an isomorphism, the natural projection $X_{T^{i}} \rightarrow X_{T}$ is an isomorphism. Furthermore, $\left(f_{T}\right)_{*}\left(M_{T^{i}}\right)$ can be identified with $\left(f_{T}\right)_{*}\left(M_{T}\right)=H^{0}\left(X_{T}, M_{T}\right)$ via $K \rightarrow K^{1 / p^{i}}$. Thus, $S_{\phi_{T}^{n}}^{0}\left(f_{T}\right)_{*}\left(M_{T}\right)$ is identified with

$$
S_{s, m}^{0}:=\operatorname{im}\left(H^{0}\left(X_{s},\left(L_{s}^{\frac{p^{m e}-1}{p^{e}-1}}\right)^{1 / p^{m e}} \otimes M_{s}\right) \rightarrow H^{0}\left(X_{s}, M_{s}\right)\right)
$$

In particular, for $m \gg 0$, the sheaf (6.8) can be identified with $S^{0}\left(X_{s}, \sigma\left(X_{s}, \phi_{s}\right) \otimes M_{s}\right)$.

\subsection{Uniform stabilization}

THEOREM 6.18. In the situation of Notation 6.1, if $L \otimes M^{p^{e}-1}$ is $f$-ample, then there is an integer $n>0$ such that for all integers $m \geqslant n$ and perfect points $s \in V$,

$$
\operatorname{im}\left(S_{\phi^{m}}^{0} f_{*}(M) \otimes_{\mathscr{O}_{V} m e} k(s)^{1 / p^{m e}} \rightarrow H^{0}\left(X_{s}, M_{s}\right)\right)=S_{s, m}^{0}=S^{0}\left(X_{s}, \sigma\left(X_{s}, \psi_{s}\right) \otimes M_{s}\right) .
$$

(Note that $\psi_{s}$ is defined in Section 2.3 and $S_{s, m}^{0}$ in Remark 6.17.)

Proof. We show the statement by induction on the dimension of $V$. There is nothing to prove if $\operatorname{dim} V=0$. Hence, we may proceed to the induction step and assume that $\operatorname{dim} V>0$. By Proposition 6.16 , there is an $n>0$ such that for every perfect point $s \in V$ and every $m \geqslant n$, we have

$$
\operatorname{im}\left(S_{\phi^{m}}^{0} f_{*}(M) \otimes_{\mathscr{O}_{V} m e} k(s)^{1 / p^{m e}} \rightarrow H^{0}\left(X_{s}, M_{s}\right)\right)=S_{s, m}^{0} .
$$

According to Lemma 2.15, by possibly increasing $n$, we may find a non-empty, dense open set $U$ such that for all $m \geqslant n$,

$$
\left.\sigma_{m}(X / V, \phi)\right|_{U}=\sigma_{n}(X / V, \phi) \times\left._{V^{n e}} V^{m e}\right|_{U} .
$$

Therefore, applying Corollary 6.12 yields (also by possibly increasing $n$ ) that for all $m \geqslant n$,

$$
V^{m e} \times\left._{V^{n e}} S_{\varphi^{n}}^{0} f_{*}(M)\right|_{U}=\left.S_{\varphi^{m}}^{0} f_{*}(M)\right|_{U} .
$$

It then follows that for every perfect point $s \in U$,

$$
\operatorname{im}\left(S_{\phi^{m}}^{0} f_{*}(M) \otimes_{\mathscr{O}_{V} m e} k(s)^{1 / p^{m e}} \rightarrow H^{0}\left(X_{s}, M\right)\right)
$$

is the same for all $m \geqslant n$. Therefore, $S_{s, m}^{0}$ is stabilized for all perfect $s \in U$ for values of $m \geqslant n$. Hence, it is equal to $S^{0}\left(X_{s}, \sigma\left(X_{s}, \psi_{s}\right) \otimes M_{s}\right)$. This shows the statement of the proposition for all perfect $s \in U$. We fix the $n_{0}=n$ used above for future use.

On the other hand, consider the reduced scheme $V_{1}=V \backslash U$. Write $V_{1}=\coprod V_{1, i}$ as a disjoint union of regular, locally closed, integral subschemes. Each $V_{1, i}$ has smaller dimension than $V$. In 


\section{Z. Patakfalvi, K. Schwede And W. Zhang}

particular, there exists an $n_{i}$ such that the statement holds for $f_{i}: X_{V_{i}} \rightarrow V_{i}$. Letting $n$ be the maximum of $n_{0}$ and the $n_{i}$, we obtain our result since every perfect point of $X$ factors through a point of $U$ or of one of the $V_{i}$.

Corollary 6.19. In the situation of Notation 6.1, if $L \otimes M^{p^{e}-1}$ is $f$-ample, then there is a dense open set $U \subseteq V$ such that for every perfect point $s \in U$,

$$
S_{\phi^{m}}^{0} f_{*}(M) \otimes_{\mathscr{O}_{V} m e} k(s)^{1 / p^{m e}}=S^{0}\left(X_{s}, \sigma\left(X_{s}, \psi_{s}\right) \otimes M_{s}\right) .
$$

In particular, $\operatorname{dim}_{k(s)} S^{0}\left(X_{s}, \sigma\left(X_{s}, \psi_{s}\right) \otimes M_{s}\right)$ is constant on an open set, and the rank of $S_{\phi^{m}}^{0} f_{*}(m)$ equals this dimension.

Proof. The main statement follows immediately from Theorem 6.18 and point (d) of Proposition 6.16. The corollary follows from basic properties of coherent sheaves [Har77, Exercise II.5.8].

If there is a point $s_{0} \in V$ such that in $X_{s_{0}}$ we have $H^{0}=S^{0}$, then we can say more than the above corollary: it turns out that it can be assumed that $s_{0} \in U$, and further that $U$ has other useful properties. This is proved in the following theorem.

TheOREM 6.20. In the situation of Notation 6.1, if $L \otimes M^{p^{e}-1}$ is $f$-ample and there is a perfect point $s_{0} \in V$ such that $H^{0}\left(X_{s_{0}}, M_{s_{0}}\right)=S^{0}\left(X_{s_{0}}, \sigma\left(X_{s_{0}}, \psi_{s_{0}}\right) \otimes M_{s_{0}}\right)$, then there is an open neighborhood $U$ of $s_{0}$, such that

(a) $\left.f_{*} M\right|_{U}$ is locally free and compatible with base change and

(b) $H^{0}\left(X_{v}, M_{v}\right)=S^{0}\left(X_{v}, \sigma\left(X_{v}, \psi_{v}\right) \otimes M_{v}\right)$ for every perfect point $v \in U$.

In particular, $\operatorname{dim} S^{0}\left(X_{v}, \sigma\left(X_{v}, \psi_{v}\right) \otimes M_{v}\right)$ is constant for $v \in U$.

Proof. By Theorem 6.18, there is an $n$ such that for every $v \in V$ and integer $m \geqslant n$, the natural base-change homomorphism induces a surjection as follows:

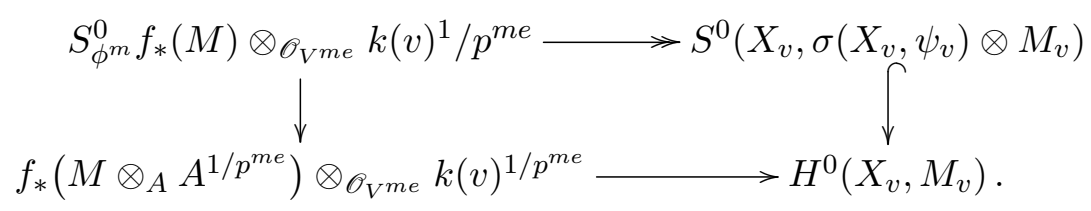

Furthermore, the right vertical arrow is surjective for $v=s_{0}$. It follows that the bottom horizontal arrow then also is. Using [Har77, Corollary III.12.11] concludes the proof of point (a).

To prove point (b), let us again consider (6.9) for $v=s_{0}$. Now, we know that the bottom horizontal arrow is an isomorphism. However, the left vertical arrow is then also surjective. By Nakayama's lemma, there is an equality $\left(S_{\phi^{n}}^{0} f_{*}(M)\right)_{v}=\left(f_{*}(M)\right)_{v}$ of stalks, which means that by possibly restricting $U$, for every $v \in U$, the left vertical arrow of (6.9) is surjective. However, then, since the bottom horizontal arrow is an isomorphism for every $v \in U$, point (b) follows.

The surjection in Proposition 6.16 is not an isomorphism in general, by the following two examples. In the first example, the global geometry of the smooth fibers causes the anomaly, while in the second, the degeneration of $F$-pure singularities to non- $F$-pure singularities is the main culprit. However, we first show a lemma.

Lemma 6.21. Let $Y$ be a smooth curve over $k$ and $G$ an effective divisor on $Y$. Define $\mathscr{B}_{Y}^{1}$ as the cokernel of $\mathscr{O}_{X} \rightarrow F_{*} \mathscr{O}_{X}$. Then

$$
S^{0}\left(Y, \sigma(X, 0) \otimes \omega_{Y}(G)\right)=H^{0}\left(Y, \omega_{Y}(G)\right) \Leftrightarrow H^{0}\left(Y, \mathscr{B}_{Y}^{1}\left(-p^{n} G\right)\right)=0 \quad \forall n>0 .
$$




\section{$F$-SINGULARITIES IN FAMILIES}

Proof. Consider the exact sequence

$$
0 \longrightarrow \mathscr{O}_{Y} \longrightarrow F_{*} \mathscr{O}_{Y} \longrightarrow \mathscr{B}_{Y}^{1} \longrightarrow 0 \text {. }
$$

Twist this sequence by $-G$, apply cohomology, and use that since $G$ is effective,

(a) either $G>0$ and hence $H^{0}\left(Y,\left(F_{*} \mathscr{O}_{Y}\right)(-G)\right) \cong H^{0}\left(\mathscr{O}_{Y}\left(-F^{*} G\right)\right)=0$,

(b) or $G=0$ and then $H^{0}\left(Y, \mathscr{O}_{Y}\right) \rightarrow H^{0}\left(Y, F_{*} \mathscr{O}_{Y}\right)$ is an isomorphism.

In either case, we have another exact sequence

$$
0 \longrightarrow H^{0}\left(Y, \mathscr{B}_{Y}^{1}(-G)\right) \longrightarrow H^{1}\left(Y, \mathscr{O}_{Y}(-G)\right) \longrightarrow H^{1}\left(Y, F_{*} \mathscr{O}_{Y}\left(-F^{*} G\right)\right),
$$

where the last map is the Serre dual to $H^{0}\left(Y,\left(F_{*} \omega_{Y}\right)(G)\right) \rightarrow H^{0}\left(Y, \omega_{Y}(G)\right)$. Furthermore, observe that the map $H^{0}\left(Y,\left(F_{*}^{n+1} \omega_{Y}\right)(G)\right) \rightarrow H^{0}\left(Y,\left(F_{*}^{n} \omega_{Y}\right)(G)\right)$ is harmlessly identified with the map $H^{0}\left(Y,\left(F_{*} \omega_{Y}\right)\left(p^{n} G\right)\right) \rightarrow H^{0}\left(Y,\left(\omega_{Y}\left(p^{n} G\right)\right)\right)$. Therefore, combining the previous statements, we see that $H^{0}\left(Y,\left(F_{*}^{n+1} \omega_{Y}\right)(G)\right) \rightarrow H^{0}\left(Y,\left(F_{*}^{n} \omega_{Y}\right)(G)\right)$ surjects if and only if $H^{0}\left(Y, \mathscr{B}_{Y}^{1}\left(-p^{n} G\right)\right)$ $=0$.

EXAMPLE 6.22. We choose two smooth projective curves $C$ and $D$ of genus 3 over an algebraically closed field $k$ of prime characteristic with two ample line bundles $N_{C}$ and $N_{D}$ (of degree 1) such that $S^{0}\left(C, \omega_{C} \otimes N_{C}\right) \neq H^{0}\left(C, \omega_{C} \otimes N_{C}\right)$ but $S^{0}\left(D, \omega_{D} \otimes N_{D}\right)=H^{0}\left(D, \omega_{D} \otimes N_{D}\right)$. Since the relative Picard scheme over the moduli stack of smooth curves of genus $g$ is smooth and irreducible, there is a (possibly reducible) curve connecting $\left(C, N_{C}\right)$ and $\left(D, N_{D}\right)$ in the above moduli space. However, by possibly replacing $\left(C, N_{C}\right)$ and $\left(D, N_{D}\right)$, we may then also find an irreducible curve connecting them. Therefore, by passing to the normalization of this curve, we may assume that there are a family $f: X \rightarrow V$, a line bundle $N$ on $X$, and two points $c, d \in C$ such that if we set $\left(C, N_{C}\right):=\left(X_{c},\left.N\right|_{X_{c}}\right)$ and $\left(C, N_{C}\right):=\left(X_{c},\left.N\right|_{X_{c}}\right)$, then

(a) $V$ is a smooth curve;

(b) $X$ is a family of smooth curves of genus 3 ;

(c) $\operatorname{deg}_{X / V} N=1$;

(d) $S^{0}\left(C, \omega_{C} \otimes N_{C}\right) \neq H^{0}\left(C, \omega_{C} \otimes N_{C}\right)$; and

(e) $S^{0}\left(D, \omega_{D} \otimes N_{D}\right)=H^{0}\left(D, \omega_{D} \otimes N_{D}\right)$.

Furthermore, fix $e=1$ and $L:=\omega_{X / V}^{1-p}$, and let $\phi: \omega_{X / V}^{1 / p} \rightarrow \mathscr{O}_{X_{V^{1}}}$ be the map with $D_{\phi}=0$ (see Definition 2.8 for the definition of $D_{\phi}$ ). Note that by Lemma 2.21, we have $D_{\phi_{s}}=0$ as well. Set $M:=\omega_{X / V} \otimes N$.

By assumption (c), for every $v \in V$ and $i>0$, we have $H^{i}\left(X_{v}, N_{v}\right)=0$. Therefore, $f_{*} N$ is a vector bundle of rank 3, and its formation is compatible with arbitrary base change. In particular, by Proposition 6.20 and assumption (e), the subsheaf $S_{\phi}^{n} f_{*}\left(\omega_{X / V} \otimes M\right) \subseteq f_{*}\left(\omega_{X / V} \otimes M\right)$ is generically isomorphic to $f_{*}\left(\omega_{X / V} \otimes M\right)$. Furthermore, since it is a subsheaf of $f_{*}\left(\omega_{X / V} \otimes M\right)$, it is torsion free, and since $V$ is a curve, it is locally free of rank 3 . Therefore, we have

$$
\operatorname{dim}_{k} S_{\phi}^{n} f_{*}\left(\omega_{X / V} \otimes M\right) \otimes k(c)=3 .
$$

However,

$$
\operatorname{dim}_{k} S^{0}\left(C, \omega_{C} \otimes M_{C}\right)<\operatorname{dim}_{k} H^{0}\left(C, \omega_{C} \otimes M_{C}\right)=3 .
$$

This shows that the base-change morphism

$$
S_{\phi^{n}}^{0} f_{*}\left(\omega_{X / V} \otimes M\right) \otimes k(c) \rightarrow S^{0}\left(C, \omega_{C} \otimes M_{C}\right)
$$




\section{Z. Patakfalvi, K. Schwede And W. Zhang}

cannot be isomorphism.

We are left with giving the polarized curves $\left(C, M_{C}\right)$ and $\left(D, M_{D}\right)$. For that, consider the situation of Lemma 6.21. By [Tan72, Lemma 12], we have $H^{0}\left(Y, \mathscr{B}^{1}\left(-p^{n} G\right)\right)=0$ if $p^{n} G \geqslant n(Y)$, where $n(Y)$ is a numerical invariant of the curve, which is at most 1 for genus 3 curves [Tan72, Lemma 10]. Hence $H^{0}\left(Y, \mathscr{B}^{1}\left(-p^{n} G\right)\right)=0$ if $\operatorname{deg} G \geqslant 1$ and $n>1$. In particular, in our special case (that is, if $Y$ is of genus 3 and $\operatorname{deg} G \geqslant 1$ ), Lemma 6.21 states that

$$
S^{0}\left(Y, \sigma(Y, 0) \otimes \omega_{Y}(G)\right)=H^{0}\left(Y, \omega_{Y}(G)\right) \Leftrightarrow H^{0}\left(Y, \mathscr{B}_{Y}^{1}(-G)\right)=0 .
$$

Finding a curve where $H^{0}\left(Y, \mathscr{B}_{Y}^{1}(-G)\right)=0$ is quite easy, because, again, using [Tan72, Lemma 12] yields that if $n(Y)=1$, then there is a degree 1 divisor $G$ for which $H^{0}\left(Y, \mathscr{B}_{Y}^{1}(-G)\right) \neq 0$, and then by passing to a linearly equivalent divisor, we may also assume that $G$ is effective. Further, [Tan72, Example 1] gives a curve $\left(x^{3} y+y^{3} z+z^{3} x=0\right)$ for which $n(Y)=1$. Therefore, we have found $C$ and $N_{C}$.

To find $D$ and $N_{D}$, assume further that $k=\overline{\mathbb{F}}_{3}$. Then by [Mil72, Kob75], a general genus 3 curve curve is ordinary or, equivalently, $S^{0}\left(Y, \sigma(Y, 0) \otimes \omega_{Y}\right)=H^{0}\left(Y, \omega_{Y}\right)$. Therefore, we have $H^{0}\left(Y, \mathscr{B}_{Y}^{1}\right)=0$ for such a $Y$. Now, take an arbitrary effective degree 1 divisor $G$. Then $\mathscr{B}_{Y}^{1}(-G)$ embeds into $\mathscr{B}_{Y}^{1}$ and hence $H^{0}\left(Y, \mathscr{B}_{Y}^{1}(-G)\right)=0$ as well. In particular, we can choose $D$ to be a generic curve and $N_{D}$ to be an arbitrary degree 1 line bundle on $D$.

EXAMPLE 6.23. In the following example, for an $f$-ample line bundle $Q$, the surjection

$$
S_{\varphi^{n}}^{0} f_{*}\left(Q^{l}\right) \otimes_{\mathscr{O}_{V^{n e}}}\left(k(s)^{1 / p^{n e}}\right) \rightarrow S^{0}\left(X_{s}, \sigma\left(X_{s}, \phi_{s}\right) \otimes Q_{s}^{l}\right)
$$

is not an isomorphism for any integer $n \geqslant n_{0}$ and $l>0$. Therefore, the isomorphism in Proposition 6.16 cannot be obtained by stronger positivity assumptions.

Let $C$ be the projective cone over a supersingular elliptic curve, and let $D$ be a non-singular cubic surface. Then these can be put into a family $f: X \rightarrow V$ as above. More precisely, we may find a family $f: X \rightarrow V$ and a point $c \in V$, such that we have $C:=X_{c}$, and hence the following hold:

(a) $V$ is a smooth curve;

(b) $X$ is a flat family of normal surfaces;

(c) $X_{c}$ is not sharply $F$-pure at one point $P \in X_{c}$; and

(d) $X_{s}$ is sharply $F$-pure for every $s \in V \backslash\{c\}$.

Let $L:=\omega_{X / V}^{1-p}$ and $\phi:=\phi_{0}$ as in the previous example. Then by Theorem 3.10 and Nakayama's lemma, $\left.\sigma_{n}(X / V, \phi)\right|_{V \backslash\{c\}} \cong \mathscr{O}_{f^{-1}(V \backslash\{c\})}$ for every $n \gg 0$. Now, choose an arbitrary sufficiently $f$-ample line bundle $M$. By Corollary 6.14 , for every $n \gg 0$, we have $\left.S_{\phi^{n}}^{0} f_{*}\left(M^{l}\right)\right|_{V \backslash\{c\}} \cong$ $\left(f_{*}(M)\right)_{V^{n e} \backslash\{c\}}$ for every integer $l>0$. In particular, we have $\operatorname{rk} S_{\phi^{n}}^{0} f_{*}\left(M^{l}\right)=\operatorname{rk} f_{*}\left(M^{l}\right)$ for every $n \gg 0$ and $l>0$ (where $n$ does not depend on $l$ ). Consequently,

$$
\operatorname{dim} S_{\phi^{n}}^{0} f_{*}\left(M^{l}\right) \otimes k(c) \geqslant \operatorname{rk} f_{*}\left(M^{l}\right)=\underbrace{\operatorname{dim}_{k} H^{0}\left(X_{c}, M_{c}^{l}\right)}_{M \text { is relatively ample enough }}
$$

On the other hand for all $n \gg 0$ (independently of $l$ ),

$$
S_{\phi^{n}}^{0} f_{*}\left(M^{l}\right) \otimes k(c) \rightarrow \underbrace{S_{\phi_{c}^{n}}^{0}\left(f_{c}\right)_{*}\left(M_{c}^{l}\right)}_{\text {by point (b) of Proposition 6.16 }}=\underbrace{H^{0}\left(X_{c}, \sigma\left(X_{c}, 0\right) \otimes M_{c}^{l}\right)}_{\text {since } M_{c} \text { is ample enough [Pat14] and Lemma 2.21 }} .
$$




\section{$F$-SINGULARITIES IN FAMILIES}

However, by the relative ampleness assumption, $M_{c}$ is globally generated; hence

$$
\operatorname{dim}_{k} H^{0}\left(X_{c}, \sigma\left(X_{c}, 0\right) \otimes M_{c}^{l}\right)<\operatorname{dim}_{k} H^{0}\left(X_{c}, M_{c}^{l}\right)
$$

(because $\left.\sigma\left(X_{c}, 0\right) \subsetneq \mathscr{O}_{X_{c}}\right)$. Therefore, (6.11) and (6.12) imply that, indeed, the surjection (6.10) is not an isomorphism for any $l>0$ and any $n \gg 0$ (bounded independently of $l$ ).

Remark 6.24. The fundamental reason for the above example is that $\sigma_{n}(X / V, \phi)$ is not flat in general.

The above two examples show that $\operatorname{dim}_{k}\left(S^{0}\left(X_{v}, \sigma\left(X_{v}, \phi_{v}\right) \otimes M_{v}\right)\right.$ is not upper semicontinuous. One might then guess that it is lower semicontinuous. The next example shows that this is also not the case (this can also be deduced from [Har98, Example 5.5] and [Tan15, Theorem 8.3] as pointed out to us by Tanaka). So, $\operatorname{dim}_{k} S^{0}\left(X_{v}, \sigma\left(X_{v}, \phi_{v}\right) \otimes M_{v}\right)$ is not semicontinuous in either direction.

ExAmple 6.25. Take a flat family $f: X \rightarrow V$ of ordinary elliptic curves and a line bundle $M$ on $X$ of relative degree 0 , such that $M_{X_{v_{0}}} \cong \mathscr{O}_{X_{v_{0}}}$ for a special $v_{0} \in V$ and $M_{X_{v}} \approx \mathscr{O}_{X_{v}}$ for generic $v \in V$. Then, by the ordinarity of the fibers, for all $v \in V$,

$$
H^{0}\left(X_{v}, M_{v}\right)=S^{0}\left(X_{v}, \sigma\left(X_{v}, 0\right) \otimes M_{v}\right) .
$$

However, the dimension $\operatorname{dim} H^{0}\left(X_{v}, M_{v}\right)$ is 1 for the special fiber and 0 for the generic one. So,

$$
\operatorname{dim} S^{0}\left(X_{v}, \sigma\left(X_{v}, 0\right) \otimes M_{v}\right)
$$

is not lower semicontinuous in this example. One can also easily modify this example by taking an $M$ with higher relative degree to obtain higher values of dimension for the generic fiber.

\subsection{Global generation and semi-positivity}

Now, suppose that $V$ is a projective variety over a perfect field $k$. In this section, we explore global generation results if $L \otimes M^{p^{e}-1}$ is ample (instead of just relatively ample). In particular, $S_{\phi^{n}}^{0} f_{*}(M)$ is globally generated for all large $n$. This should not be surprising since $S_{\phi^{n}}^{0} f_{*}(M)$ lives on $V^{n e}$, where ampleness is amplified.

Proposition 6.26. In the situation of Notation 6.1, if $V$ is projective over a perfect field $k$ and $L \otimes M^{p^{e}-1}$ is ample, then $S_{\phi^{n}}^{0} f_{*}(M)$ is globally generated for every $n \gg 0$.

Proof. Choose a globally generated ample divisor $H$ on $V$, and let $d:=\operatorname{dim} V$. Since $S_{\phi^{n}}^{0} f_{*}(M)$ is defined as the image of

$$
f_{*}\left(\left(L^{\frac{p^{n e}-1}{p^{e}-1}}\right)^{1 / p^{n e}} \otimes_{R} M\right),
$$

it is enough to show that this sheaf is globally generated as an $\mathscr{O}_{V^{n e}}$-module. By Mumford's criterion [Laz04a, Theorem 1.8.5], it is enough to show that for every $i>0$ and for the divisor $H_{n e}$ on $V^{n e}$ identified with $H$ via the isomorphism $V^{n e} \cong V$,

$$
H^{i}\left(V^{n e}, \mathscr{O}_{V^{n e}}\left(-i H_{n e}\right) \otimes_{\mathscr{O}_{V} e} f_{*}\left(\left(L^{\frac{p^{n e}-1}{p^{e}-1}}\right)^{1 / p^{n e}} \otimes_{R} M\right)\right)=0 .
$$

By the relative Serre vanishing theorem for $i>0$,

$$
R^{i} f_{*}\left(\left(L^{\frac{p^{n e}-1}{p^{e}-1}}\right)^{1 / p^{n e}} \otimes_{R} M\right)=0
$$




\section{Z. Patakfalvi, K. Schwede And W. Zhang}

In particular, then to prove (6.13), it is enough to show

$$
H^{i}\left(X, L^{\frac{p^{n e}-1}{p^{e}-1}} \otimes M^{p^{n e}}\left(-i f^{*} H\right)\right)=0 .
$$

However, this is equivalent to showing

$$
H^{i}\left(X,\left(L \otimes M^{\left(p^{e}-1\right)}\right)^{\frac{p^{n e}-1}{p^{e}-1}} \otimes M\left(-i f^{*} H\right)\right)=0,
$$

which holds by the Serre vanishing theorem for $n \gg 0$ and $i>0$.

We recall the following definition.

Definition 6.27 ([Vie95, Definition 2.11]). Let $\mathscr{F}$ be a sheaf on a normal, quasi-projective (over a perfect field $k$ ) variety $V$ and $U \subseteq V$ a dense open set. Let $U_{\text {lf }}$ be the open locus of $V$ where $\mathscr{F}^{\prime}:=\mathscr{F} /($ torsion) is locally free. Then $\mathscr{F}$ is weakly positive over $U$ if for a fixed (or, equivalently, every [Vie95, Lemma 2.14(a)]) ample line bundle $\mathscr{H}$ for every $a>0$, there is a $b>0$ such that $S^{\langle a b\rangle}(\mathscr{F}) \otimes \mathscr{H}^{b}$ is globally generated over $U \cap U_{\text {lf }}$ (here $S^{\langle a b\rangle}(\mathscr{F})$ denotes the abth symmetric reflexive power of $\mathscr{F})$. We say that $\mathscr{F}$ is weakly positive if it is weakly positive over some dense open set.

Lemma 6.28. If $g: Y \rightarrow Z$ is a finite morphism of normal varieties, quasi-projective over $k$, $U \subseteq Z$ a dense open set, and $\mathscr{F}$ a sheaf on $Z$, then $\mathscr{F}$ is weakly positive over $U$ if and only if $g^{*} \mathscr{F}$ is weakly positive over $g^{-1}(U)$.

Proof. The "only if" direction is shown in [Vie95, Lemma 2.15.1]. For the other direction, according to Definition 6.27, by throwing out a codimension 2 subset, we may assume that $\mathscr{F}$ is locally free and $Y$ is flat over $Z$. In particular, $g^{*} \mathscr{F}$ is then also locally free. Choose a very ample divisor $\mathscr{H}$ on $Z$ such that

(a) $\mathscr{H} \mathrm{om}\left(g_{*} \mathscr{O}_{Y}, \mathscr{H}\right)$ is globally generated and

(b) $g_{*} \mathscr{O}_{Y} \otimes \mathscr{H}$ is globally generated.

Then, fix an $a>0$. By the weak positivity of $g^{*} \mathscr{F}$, there is a $b>0$ such that there is a homomorphism $\alpha: \mathscr{O}_{Y}^{\oplus N} \rightarrow S^{\langle a b\rangle}\left(g^{*} \mathscr{F}\right) \otimes g^{*} \mathscr{H}^{b}$ surjective over $g^{*} U$. Then, consider for every choice of $s \in \operatorname{Hom}\left(g_{*} \mathscr{O}_{Y}, \mathscr{H}\right)$ and $s^{\prime} \in H^{0}\left(\mathscr{H}^{b-1}\right)$ the following composition:

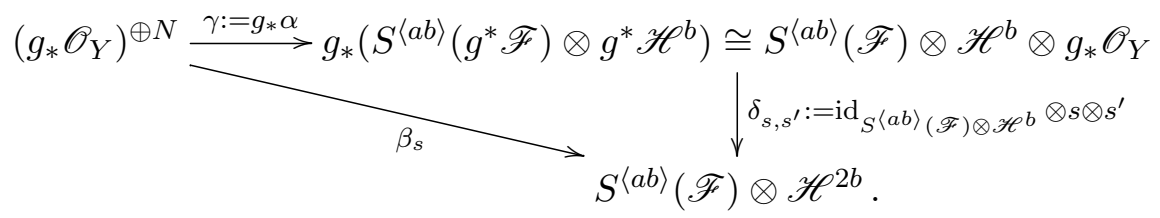

Choose a point $P \in U$ and an element $f$ of the fiber $S^{\langle a b\rangle}(\mathscr{F}) \otimes k(P)$ over $P$. Then, by assumption (b), for any preimage $Q$ of $P$, there is a section $t^{\prime} \in \mathscr{O}_{Y}^{\oplus N}$ such that $\alpha\left(t^{\prime}\right)_{Q}=$ $g^{*} f \times$ "generator". The section $t^{\prime}$ descends to a section $t:=g_{*}\left(t^{\prime}\right) \in\left(g_{*} \mathscr{O}_{Y}\right)^{\oplus N}$ such that $\gamma(t)_{P}=f \times$ "generator" $\times h$ for some $h \in\left(g_{*} \mathscr{O}_{Y}\right)_{P}$. However, then by assumption (a) and the very ampleness of $\mathscr{H}$ for a suitable choice of $s$ and $s^{\prime}$, we have that $\left.\delta_{s, s^{\prime}}(\gamma(t))\right)_{P}=f \times$ "generator" is not zero at $P$. Therefore, for every point $P$ in $U$ and every element $f$ in the fiber of $S^{\langle a b\rangle}(\mathscr{F}) \otimes \mathscr{H}^{2 b}$ at that point, we find a section of $S^{\langle a b\rangle}(\mathscr{F}) \otimes \mathscr{H}^{2 b}$ whose image in the fiber is $f$ up to multiplication by unit. This finishes our proof.

Corollary 6.29. In the situation of Notation 6.1, let $V$ be projective over a prefect field $k$, $L \otimes M^{p^{e}-1}$ ample, and let $n>0$ be an integer such that there is an open set $U \subseteq V$ for which

$$
S_{\phi^{n}}^{0} f_{*}(M) \times_{U^{n e}} U^{m e}=\left.S_{\phi^{m}}^{0} f_{*}(M)\right|_{U^{m e}}
$$




\section{$F$-SINGULARITIES IN FAMILIES}

for every integer $m \geqslant n$. Then $S_{\phi^{n}}^{0} f_{*}(M)$ is weakly positive. Note that an integer as above always exists by Corollary 6.12 and Proposition 5.2.

Proof. By the assumption, there is an embedding

$$
S_{\phi^{m}}^{0} f_{*}(M) \hookrightarrow S_{\phi^{n}}^{0} f_{*}(M) \times_{V^{n e}} V^{m e}
$$

which is generically an isomorphism. Since the left sheaf is globally generated and hence weakly positive, so is the right one. However, then by Lemma 6.28, so is $S_{\phi^{n}}^{0} f_{*}(M)$.

Lemma 6.30. If $\mathscr{F}$ is a coherent sheaf on a normal variety $V$ over a perfect field $k$, then $\mathscr{F}$ is weakly positive if and only if for every ample line bundle $\mathscr{H}$ and every integer $p \nmid r>0$, there is a finite morphism $\tau: T \rightarrow V$ such that $\tau^{*} \mathscr{H} \cong\left(\mathscr{H}^{\prime}\right)^{r}$ for some line bundle $\mathscr{H}^{\prime}$ and $\tau^{*} \mathscr{F} \otimes \mathscr{H}^{\prime}$ is weakly positive.

Proof. The proof is identical to the " $(b) \Rightarrow(a)$ " part of [Vie95, Lemma 2.15.1].

THEOREM 6.31. In the situation of Notation 6.1, if $V$ is projective and $L \otimes M^{p^{e}-1}$ is nef and $f$-ample, then $S_{\phi^{n}}^{0} f_{*}(M)$ is weakly positive for $n \gg 0$.

Proof. Choose an integer $n>0$ as in Corollary 6.29. Fix an ample line bundle $\mathscr{H}$ on $V$, an integer $p \nmid r>0$, and a finite morphism $\tau: T \rightarrow V$, such that $\tau^{*} \mathscr{H} \cong\left(\mathscr{H}^{\prime}\right)^{r}$ for some line bundle $\mathscr{H}^{\prime}$. Such a morphism exits by [Vie95, Lemma 2.1]. By Lemma 6.30, we are supposed to prove that $\left(\tau^{1 / p^{n e}}\right)^{*} S_{\phi}^{n} f_{*}(M) \otimes_{\mathfrak{O}_{V} \text { ne }}\left(\mathscr{H}^{\prime}\right)^{1 / p^{n e}}$ is weakly positive. By disregarding codimension 2 closed sets, we may assume that $T$ is regular as well. Then by point (c) of Proposition 6.16, we see that $n$ also satisfies the assumptions of Corollary 6.29, but for $f$ and $M$ replaced by $f_{T}$ and $M_{T} \otimes f_{T}^{*} \mathscr{H}_{T}^{\prime}$, respectively. In particular, since $M_{T} \otimes f_{T}^{*} \mathscr{H}_{T}^{\prime}$ is ample,

$$
S_{\phi_{T}^{n}}^{0}\left(f_{T}\right)_{*}\left(M_{T} \otimes f_{T}^{*} \mathscr{H}_{T}^{\prime}\right) \cong \mathscr{H}_{T}^{\prime} \otimes S_{\phi_{T}^{n}}^{0}\left(f_{T}\right)_{*}\left(M_{T}\right)
$$

is weakly positive over $T^{\text {ne }}$.

\subsection{Relation to global canonical systems}

Another subsheaf $S^{0} f_{*}(\sigma(X, \Delta) \otimes M)$ of $f_{*}(M)$ has been introduced in [HX15, Definition 2.14] with a definition similar to that of $S_{\phi^{n}}^{0} f_{*}(M)$. In this section, we show some of the similarities and differences between the two sheaves. The advantage of $S^{0} f_{*}(\sigma(X, \Delta) \otimes M)$ over $S_{\phi^{n}}^{0} f_{*}(M)$ is that it lives on one $V$, there is no involvement of $V^{\text {ne }}$ at all. On the other hand, we show that contrary to $S_{\phi^{n}}^{0} f_{*}(M)$, it does not restrict even generically to $S^{0}\left(X_{s}, \sigma\left(X_{s}, \Delta_{s}\right) \otimes M_{s}\right)$.

Throughout the section, we use the divisorial language since the presentation seems to be more straightforward this way; hence $\Delta$ satisfies the conditions of Remark 2.11. Recall that we defined the notion of pair in our setting in Definition 2.12. Whenever we say pair, we mean everything assumed there. In particular, this includes all the assumptions on $f: X \rightarrow V$ from Notation 2.1: flatness, equidimensionality, etc.

First, we recall the definition of $S^{0} f_{*}(M)$.

Definition 6.32 ([HX15, Definition 2.14]). Let $\Delta$ be an effective $\mathbb{Q}$-divisor such that $(X, \Delta)$ is a pair and assume that $f: X \rightarrow V$ is projective. Let $e$ be the least ${ }^{9}$ positive integer such that $\left(p^{e}-1\right)\left(K_{X}+\Delta\right)$ is Cartier. Then given a line bundle $M$ on $X$, we define

$$
S^{0} f_{*}(\sigma(X, \Delta) \otimes M):=\bigcap_{n>0} \operatorname{im}\left(f_{*}\left(F_{*}^{n e} \mathscr{O}_{X}\left(\left(1-p^{n e}\right)\left(K_{X}+\Delta\right)\right) \otimes M\right) \rightarrow f_{*}(M)\right) .
$$

${ }^{9}$ The intersection in the definition is easily seen to be descending; hence if one chooses another $e$, the same object results. 


\section{Z. Patakfalvi, K. Schwede And W. Zhang}

We say that $S^{0} f_{*}(\sigma(X, \Delta) \otimes M)$ stabilizes if the above intersection stabilizes. Note that in that case, $S^{0} f_{*}(\sigma(X, \Delta) \otimes M)$ is a coherent sheaf.

Recall that $S^{0} f_{*}(\sigma(X, \Delta) \otimes M)$ stabilizes if $M-K_{X}-\Delta$ is relatively ample; see [HX15, Proposition 2.15] for a proof.

Proposition 6.33. Let $\Delta$ be an effective $\mathbb{Q}$-divisor such that $(X, \Delta)$ is a pair and assume that $f: X \rightarrow V$ is projective. Let $M$ be a line bundle on $X$ such that $S^{0} f_{*}(\sigma(X, \Delta) \otimes M)$ stabilizes. Further assume that $V$ is regular. Then, for every $n \gg 0$,

$$
S_{\Delta, n e}^{0} f_{*}(M) \subseteq\left(S^{0} f_{*}(\sigma(X, \Delta) \otimes M)\right)_{V^{n e}}
$$

as subsheaves of $\left(f_{*}(M)\right)_{V^{n e}}$, where $S_{\Delta, n e}^{0} f_{*}(M)$ is defined in Definition 6.4.

Proof. The statement is local over the base; hence, we can assume that $V$ is affine, $K_{V} \sim 0$, and that $\mathscr{H}_{\mathrm{om}_{\mathscr{O}_{V}}}\left(F_{*}^{i} \mathscr{O}_{V}, \mathscr{O}_{V}\right) \cong F_{*}^{i} \mathscr{O}_{V}$ is a free $\mathscr{O}_{V}$-module for all $i \geqslant 0$. Note, then, that we can also identify $K_{X / V}$ with $K_{X}$. We introduce the notation

$$
L:=\mathscr{O}_{X}\left(\left(1-p^{n e}\right)\left(K_{X}+\Delta\right)\right) .
$$

We have the evaluation-at-1 map $F_{*}^{n e} \mathscr{O}_{V} \cong \mathscr{H} \mathrm{om}_{\mathscr{O}_{V}}\left(F_{*}^{n e} \mathscr{O}_{V}, \mathscr{O}_{V}\right) \rightarrow \mathscr{O}_{V}$, which we identify with the trace of $V$ based on our previous assumption $\omega_{V} \cong \mathscr{O}_{V}$. This map pulls back to $X$ to provide us with a map which we also denote by $\operatorname{Tr}_{F_{V}}^{n e}: \mathscr{O}_{X_{V} e} \cong \mathscr{O}_{X} \otimes f^{*} F_{*}^{n e} \mathscr{O}_{V} \rightarrow \mathscr{O}_{X} \otimes_{\mathscr{O}_{X}} f^{*} \mathscr{O}_{V} \cong \mathscr{O}_{X}$. Then there is a diagram as follows, which is commutative up to multiplication by a unit; cf. [Sch09, Lemma 3.9]:

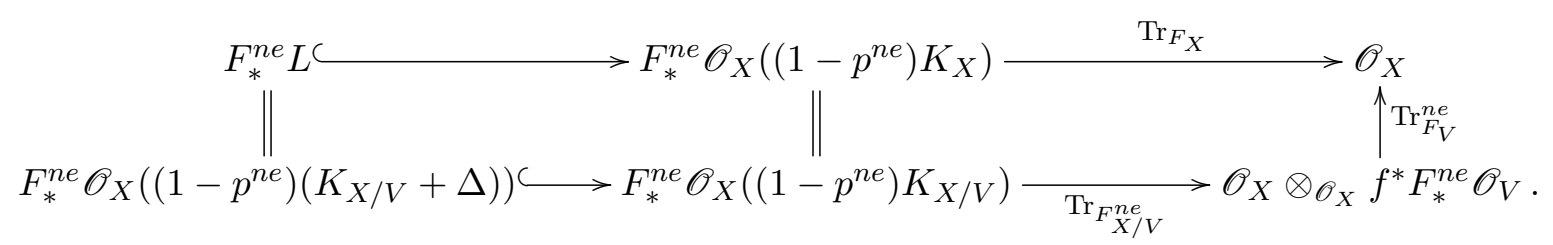

Applying the functor $f_{*}\left(\otimes_{\mathscr{O}_{X}} M\right)$ to this diagram, we obtain the following:

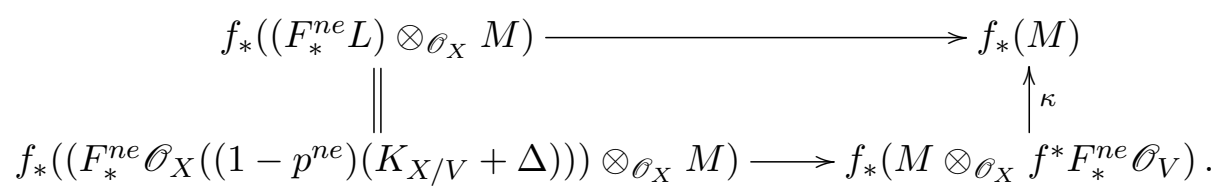

Note that for every $n \gg 0$, the image of the top horizontal row is $S^{0} f_{*}(\sigma(X, \Delta) \otimes M)$ and for every $n>0$, the image of the bottom horizontal row is $S_{\Delta, n}^{0} f_{*}(M)$. Hence for every $n \gg 0$,

$$
S^{0} f_{*}(\sigma(X, \Delta) \otimes M)=\operatorname{im}\left(S_{\Delta, n}^{0} f_{*}(M) \rightarrow f_{*}(M)\right),
$$

where the map is induced by $\operatorname{Tr}_{F_{V}}$.

Since we want containment for subsheaves of $\left(f_{*}(M)\right)_{V^{n e}}$, we localize at a point of $V$. By setting $B:=\Gamma\left(V, \mathscr{O}_{V}\right), P:=f_{*}(M)$, and $Q:=F_{V, *}^{n e}\left(S_{\Delta, n}^{0} f_{*}(M)\right)$, we are in the following situation: if $Q$ is a $B^{1 / p^{n e}}$-submodule of $B^{1 / p^{n e}} \otimes_{B} P$ for some $B$-module $P$, we would like to show that $Q \subseteq$ $\theta(Q) \otimes_{B} B^{1 / p^{n e}}$, where $\theta \in \operatorname{Hom}_{B}\left(B^{1 / p^{n e}}, B\right)$ is the generator. This is simply Lemma 3.20.

EXAMPLE 6.34. We provide an example where the two sheaves of Proposition 6.33 have different ranks for every $n \gg 0$. Fix an algebraically closed field $k$ of characteristic $p>0$. Let $X:=$ $\mathbb{P}^{1} \times \mathbb{A}^{1}, U=\mathbb{A}^{1} \times \mathbb{A}^{1}=\operatorname{Spec} k[y, x] \subseteq X$, and $V:=\mathbb{A}^{1}:=\operatorname{Spec} k[x]$. Define the divisor $\Delta:=(1 /(p-1)) V\left(y^{p}-x\right)$, which is a priori a divisor in $U$, but it happens to have a support 


\section{$F$-SINGULARITIES IN FAMILIES}

which is a closed subvariety of $X$ as well. Note that $V\left(y^{p}-x\right)$ is a subvariety of $X$ isomorphic to $\mathbb{A}^{1}$. In particular, $(X, \Delta)$ is sharply $F$-pure. Now, choose a line bundle $N$ on $X$ which is relatively ample over $V$, and let $M:=N^{l}$ for some $l \gg 0$. Then by [HX15, Lemma 2.19], we have $S^{0} f_{*}(\sigma(X, \Delta) \otimes M)=f_{*}(M)$. Therefore,

$$
\left(S^{0} f_{*}(\sigma(X, \Delta) \otimes M)\right)_{V^{n e}}=\left(f_{*}(M)\right)_{V^{n e}} .
$$

On the other hand, by Proposition 6.5, we have $S_{\Delta, n}^{0} f_{*}(M) \subseteq f_{*}\left(\sigma_{n}(X / V, \Delta) \otimes M\right)$. By Theorem 3.10 and Lemma 2.21, for every $n \gg 0$ and $s \in V$, we have $\left.\sigma_{n}(X / V, \Delta)\right|_{X_{s}}=\sigma\left(X_{s}, \Delta\right)$. However, $\left.\Delta\right|_{X_{s}}$ is one point with multiplicity $p /(p-1)$ and hence not sharply $F$-pure. In particular, $\sigma\left(X_{s}, \Delta\right) \neq \mathscr{O}_{X_{s}}$ for every $s \in S$. Hence, for every $n \gg 0$, we have $\left.\sigma_{n}(X / V, \Delta)\right|_{X_{s}} \subsetneq \mathscr{O}_{X_{s}}$. It follows by the relative ampleness of $M$ that $f_{*}\left(\sigma_{n}(X / V, \Delta) \otimes M\right)$ has smaller rank than $f_{*}(M)$. Then $S_{\Delta, n}^{0} f_{*}(M)$ has also smaller rank than $\left(S^{0} f_{*}(\sigma(X, \Delta) \otimes M)\right)_{V^{n e}}$ by $(6.14)$.

Example 6.34 has an important corollary, which follows immediately from point (d) of Proposition 6.16 and Theorem 6.18.

Corollary 6.35. In general, $S^{0} f_{*}(\sigma(X, \Delta) \otimes M) \otimes k(s)$ is not isomorphic to $S^{0}\left(X_{s}, \sigma\left(X_{s}, \Delta_{s}\right) \otimes\right.$ $\left.M_{s}\right)$. In fact, there are examples when the former has strictly bigger dimension than the latter for every closed point $s \in V$.

Compare the following proposition with Proposition 3.23.

Proposition 6.36. Let $\Delta$ be an effective $\mathbb{Q}$-divisor such that $(X, \Delta)$ is a pair, and assume that $f: X \rightarrow V$ is projective. Let $M$ be a line bundle on $X$, and assume that $V$ is regular. Then for every $n \geqslant m \geqslant 0$,

$$
S^{0} f_{V^{n e}, *}\left(\sigma\left(X_{V^{n e}}, \Delta_{V^{n e}}\right) \otimes M_{V^{n e}}\right) \subseteq\left(S^{0} f_{V^{m e}, *}\left(\sigma\left(X_{V^{m e}}, \Delta_{V^{m e}}\right) \otimes M_{V^{m e}}\right)\right)_{V^{n e}}
$$

as subsheaves of $\left(f_{*}(M)\right)_{V^{n e}}$. Furthermore, if these two sheaves stabilize, then

$$
\begin{aligned}
& \left.\kappa\left(F_{V, *}^{(n-m) e} S^{0} f_{V^{n e}, *}\left(\sigma\left(X_{V^{n e}}, \Delta_{V^{n e}}\right) \otimes f^{*} \mathscr{O}_{V}\left(\left(1-p^{(n-m) e}\right) K_{V^{n e}}\right)\right) \otimes M_{V^{n e}}\right)\right) \\
& \quad=S^{0} f_{V^{m e}, *}\left(\sigma\left(X_{V^{m e}}, \Delta_{V^{m e}}\right) \otimes M_{V^{m e}}\right),
\end{aligned}
$$

where $\kappa$ is induced by $\operatorname{Tr}_{V}^{(n-m) e}$.

Proof. Note that by replacing $f: X \rightarrow V$ by $f_{V^{m e}}: X_{V^{m e}} \rightarrow V^{m e}$, we may assume that $m=0$, and then by replacing $e$ by $(n-m) e$ that $n=1$. As in the previous proof, let us assume that $V$ is affine, $K_{V} \sim 0$, and that $\mathscr{H}_{\mathrm{om}_{\mathscr{O}_{V}}}\left(F_{*}^{e} \mathscr{O}_{V}, \mathscr{O}_{V}\right) \cong F_{*}^{e} \mathscr{O}_{V}$ is a free $\mathscr{O}_{V}$-module. Consider the following commutative diagram:

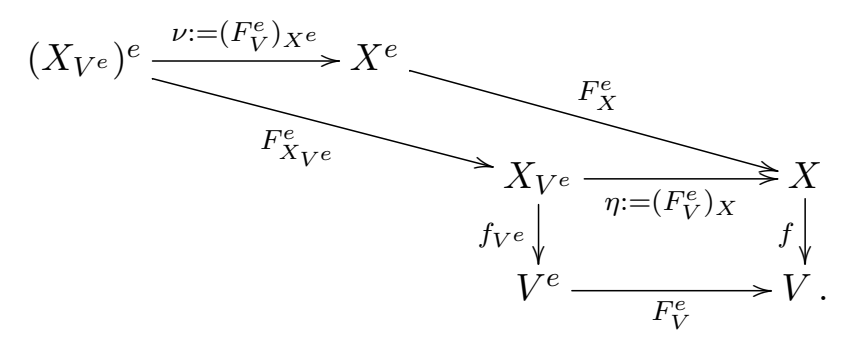

We fix the notation

$$
\mathscr{L}_{\Delta}^{\prime}:=\mathscr{O}_{X_{V^{e}}}\left(\left(1-p^{e}\right)\left(K_{X_{V^{e}}}+\eta^{*} \Delta\right)\right), \quad \mathscr{L}_{\Delta}:=\mathscr{O}_{X}\left(\left(1-p^{e}\right)\left(K_{X}+\Delta\right)\right) .
$$

Then we have

$$
\left(1-p^{e}\right) K_{X_{V^{e}}}=\left(1-p^{e}\right)\left(\eta^{*} K_{X / V}+f_{V^{e}}^{*} K_{V^{e}}\right), \quad\left(1-p^{e}\right) K_{X}=\left(1-p^{e}\right)\left(K_{X / V}+f^{*} K_{V}\right) .
$$




\section{Z. Patakfalvi, K. Schwede And W. Zhang}

Therefore,

○ applying pullback of $\operatorname{Tr}_{F_{V}^{e}}$ yields a homomorphism $\nu_{*} \mathscr{L}_{\Delta}^{\prime} \rightarrow \mathscr{L}_{\Delta}$, which then induces a homomorphism $\eta_{*} F_{X_{V}, *}^{e} \mathscr{L}_{\Delta}^{\prime} \cong F_{X, *}^{e} \nu_{*} \mathscr{L}_{\Delta}^{\prime} \rightarrow F_{X, *}^{e} \mathscr{L}_{\Delta}$;

$\circ$ applying $\operatorname{Tr}_{F_{X_{V}}^{e}}$ yields a homomorphism $F_{X_{V} e, *}^{e} \mathscr{L}_{\Delta}^{\prime} \rightarrow \mathscr{O}_{X_{V} e}$;

$\circ$ applying $\operatorname{Tr}_{F_{X}^{e}}$ yields a homomorphism $F_{X, *}^{e} \mathscr{L}_{\Delta} \rightarrow \mathscr{O}_{X}$;

○ by the assumption $K_{V} \sim 0$, the trace map $\operatorname{Tr}_{F_{V}^{e}}$ corresponds to a homomorphism $F_{V, *}^{e} \mathscr{O}_{V} \rightarrow$ $\mathscr{O}_{V}$ generating $\operatorname{Hom}_{\mathscr{O}_{V}}\left(F_{V, *}^{e} \mathscr{O}_{V}, \mathscr{O}_{V}\right)$; and

$\circ$ the previous homomorphism also induces a pullback homomorphism $\eta_{*} \mathscr{O}_{X_{V} e} \rightarrow \mathscr{O}_{X}$.

Furthermore, the above homomorphisms fit into the following diagram:

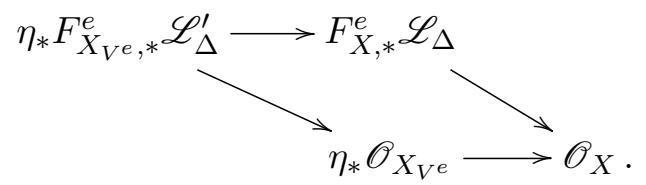

The diagram is a composition of trace maps (restricted to smaller domains determined by $\Delta$ ); hence it is commutative. Now, applying $f_{*}\left(\_\otimes M\right)$ to the above diagram and using the projection formula, we obtain the commutative diagram

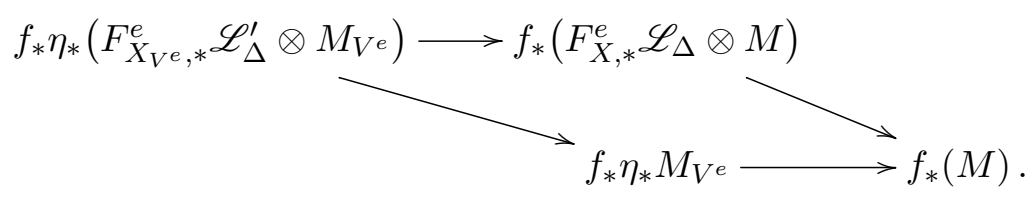

Observing that $f_{*} \eta_{*}=F_{V, *}^{e} f_{V^{e}, *}$ yields another commutative diagram:

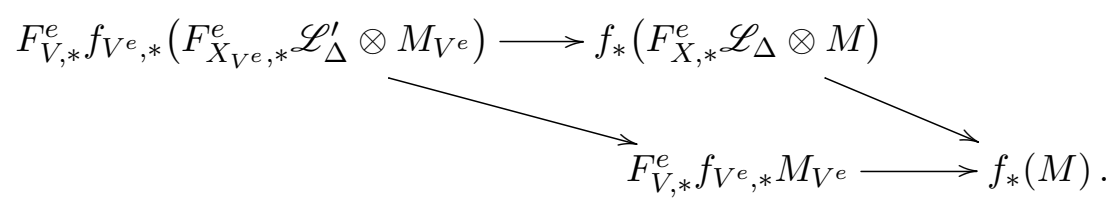

Now, note that the top horizontal arrow is split, because it is induced from $\nu_{*} \mathscr{L}_{\Delta}^{\prime} \rightarrow \mathscr{L}_{\Delta}$, which is split as well. Therefore, if we define $P:=f_{*}(M)$ and

$$
S:=\operatorname{im}\left(f_{*}\left(F_{X, *}^{e} \mathscr{L}_{\Delta} \otimes M\right) \rightarrow f_{*}(M)\right), \quad Q:=\operatorname{im}\left(f_{V^{e}, *}\left(F_{X_{V^{e}, *}}^{e} \mathscr{L}_{\Delta}^{\prime} \otimes M_{V^{e}}\right) \rightarrow f_{V^{e}, *} M_{V^{e}}\right),
$$

then we have $Q \subseteq P \otimes_{A} A^{1 / p^{e}}, S \subseteq P$, and $\left(\operatorname{id}_{P} \otimes \operatorname{Tr}_{F_{V}^{e}}\right)(Q)=S$. Therefore, by Lemma 3.20, we have $Q \subseteq S \otimes_{A} A^{1 / p^{e}}$. Since this holds for any $n$, the statement of the proposition follows.

Proposition 6.37. Let $\Delta$ be an effective $\mathbb{Q}$-divisor such that $(X, \Delta)$ is a pair, and assume that $f: X \rightarrow V$ is projective and $V$ is regular. Let $M$ be a line bundle on $X$. Then for every $n \gg 0$,

$$
S^{0} f_{V^{n e}, *}\left(\sigma\left(X_{V^{n e}}, \Delta_{V^{n e}}\right) \otimes M_{V^{n e}}\right) \subseteq S_{\Delta, n e}^{0} f_{*}(M)
$$

as subsheaves of $\left(f_{*}(M)\right)_{V^{n e}}$.

Proof. As before, let us assume that $V$ is affine, $K_{V} \sim 0$, and that $\mathscr{H} \mathrm{om}_{\mathscr{O}_{V}}\left(F_{*}^{i} \mathscr{O}_{V}, \mathscr{O}_{V}\right) \cong F_{*}^{i} \mathscr{O}_{V}$ is a free $\mathscr{O}_{V}$-module for all $i \geqslant 0$. By Lemmas 3.12 and 3.15, there is a commutative diagram

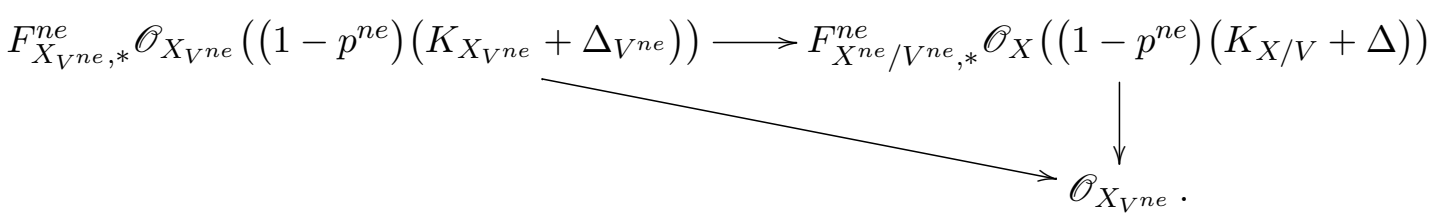




\section{$F$-SINGULARITIES IN FAMILIES}

Furthermore, the horizontal arrow in this diagram is split surjective. Therefore, after applying $f_{V^{n e}, *}\left(\_M_{V^{n e}}\right)$, the image of the vertical map still agrees with the image of the diagonal map. The former is exactly $S_{\Delta, n}^{0} f_{*}(M)$, while the latter contains $S^{0} f_{V^{n e}, *}\left(\sigma\left(X,(\Delta)_{V^{n e}}\right) \otimes M\right)$, since it is one of the terms in the intersection defining $S^{0} f_{V^{n e}, *}\left(\sigma\left(X,(\Delta)_{V^{n e}}\right) \otimes M\right)$.

Remark 6.38. Assuming that $f: X \rightarrow V$ is projective and $M-K_{X}-\Delta$ is ample, it would be natural to ask whether $\operatorname{im}\left(S^{0} f_{V^{n e}, *}\left(\sigma\left(X_{V^{n e}, \Delta_{V} n e} \otimes M_{V^{n e}}\right) \otimes M_{V^{n e}}\right) \rightarrow f_{V^{n e}, M_{s}}\right)$ equals $S^{0}\left(X_{s}, \sigma\left(X_{s}, \Delta_{s}\right) \otimes M_{s}\right)$ for all perfect points $s \in V$, in analogy with Corollary 3.22. We suspect that this is true but will not try to prove it here.

Corollary 6.39. Let $f:(X, \Delta) \rightarrow V$ be a projective morphism from a pair with $V$ regular and projective over a perfect field $k$. Further, suppose that $M$ is a line bundle on $X$ such that $M-K_{X / V}-\Delta$ is nef and $f$-ample (here $M$ denotes a Cartier divisor corresponding to $M$ ) and that rk $S^{0} f_{*}(\sigma(X, \Delta) \otimes M)$ equals the general value of $H^{0}\left(X_{s}, \sigma\left(X_{s}, \Delta_{s}\right) \otimes M_{s}\right)$. Then the sheaf $S^{0} f_{*}(\sigma(X, \Delta) \otimes M)$ is weakly positive. In particular, if $V$ is a smooth curve, then it is a nef vector bundle.

Proof. From the assumption that the rank $\operatorname{rk} S^{0} f_{*}(\sigma(X, \Delta) \otimes M)$ equals the general value of $H^{0}\left(X_{s}, \sigma\left(X_{s}, \Delta_{s}\right) \otimes M_{s}\right)$ follows that the inclusion of Proposition 6.33 is generically an isomorphism for every $n \gg 0$. However, since $S_{\phi^{n}}^{0} f_{*}(M)$ is weakly positive for every $n \gg 0$ by Theorem 6.31, we obtain by the above generically isomorphic inclusion that $\left(S^{0} f_{*}(\sigma(X, \Delta) \otimes\right.$ $M))_{V^{n e}}$ is also weakly positive. Then the weak positivity of $S^{0} f_{*}(\sigma(X, \Delta) \otimes M)$ follows from Lemma 6.28.

\section{Appendix. Relative Serre's condition}

Here we collect the statements of [HK04] and other sources that are important for the current paper for ease of reference. In some cases, we also state them in the greater generality that we need. All schemes are Noetherian and excellent and possess dualizing complexes, and all maps are separable.

Definition A.1. Let $r>0$ be an integer. A coherent sheaf $\mathscr{E}$ on a Noetherian scheme $X$ is $S_{r}$ if for every $x \in X$,

$$
\operatorname{depth}_{\mathscr{O}_{X, x}} \mathscr{E}_{x} \geqslant \min \left\{r, \operatorname{dim}_{\mathscr{O}_{X, x}} \mathscr{E}_{x}\right\} .
$$

The sheaf $\mathscr{E}$ is said to have full support if Supp $\mathscr{E}=X$. It is reflexive if the natural map

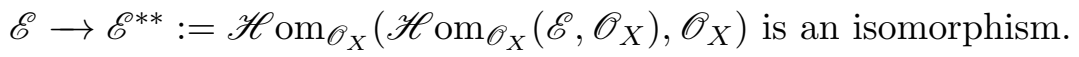

Definition A.2. If $f: X \rightarrow V$ is a morphism of Noetherian schemes and $\mathscr{E}$ is a coherent sheaf on $X$, flat over $V$, then $\mathscr{E}$ is $S_{r}$ over $V$ if $\left.\mathscr{E}\right|_{X_{v}}$ is $S_{r}$ for every $v \in V$. That is, for every $x \in X$,

$$
\operatorname{depth}_{\mathscr{O}_{X_{f(x)}}}\left(\left.\mathscr{E}\right|_{X_{f(x)}}\right)_{x} \geqslant \min \left\{r, \operatorname{dim}_{\mathscr{O}_{X_{f(x)}}}\left(\left.\mathscr{E}\right|_{X_{f(x)}}\right)_{x}\right\} \text {. }
$$

We now recall two results from [Gro67] about how depth and dimension behave in families.

Proposition A.3 ([Gro67, Proposition 6.3.1]). Let $\phi: A \rightarrow B$ be a local homomorphism of Noetherian local rings, $k$ the residue field of $A$, and $M$ and $N$ finite $A$ - and $B$-modules, respectively. If $N$ is a flat non-zero $A$-module, then

$$
\operatorname{depth}_{B}\left(M \otimes_{A} N\right)=\operatorname{depth}_{A}(M)+\operatorname{depth}_{B \otimes_{A} k}\left(N \otimes_{A} k\right) .
$$




\section{Z. Patakfalvi, K. Schwede And W. Zhang}

Proposition A.4 ([Gro67, Corollaire 6.1.2]). Let $\phi: A \rightarrow B$ be a local homomorphism of Noetherian local rings, $k$ the residue field of $A$, and $M$ and $N$ non-zero finite $A$ - and $B$-modules, respectively. If $N$ is a flat $A$-module, then

$$
\operatorname{dim}_{B}\left(M \otimes_{A} N\right)=\operatorname{dim}_{A}(M)+\operatorname{dim}_{B \otimes_{A} k}\left(N \otimes_{A} k\right) .
$$

From these we obtain the following corollary.

Corollary A.5. Let $f: X \rightarrow V$ be a morphism of Noetherian schemes and $\mathscr{E}$ a coherent sheaf on $X$ flat over $V$; then $\mathscr{E}$ is $S_{r}$ over $V$ if and only if for every $x \in X$,

$$
\begin{aligned}
\min \left\{i \mid H_{x}^{i}(\mathscr{E}) \neq 0\right\} \geqslant & \operatorname{depth}_{\mathscr{O}_{V, v}} \mathscr{O}_{V, v}+\min \left\{r, \operatorname{dim} \mathscr{E}_{x}-\operatorname{dim} \mathscr{O}_{V, v}\right\} \\
& \left(=\operatorname{depth}_{\mathscr{O}_{V, v}} \mathscr{O}_{V, v}+\min \left\{r, \operatorname{dim}\left(\mathscr{E}_{X_{v}}\right)_{x}\right\}\right),
\end{aligned}
$$

where $v:=f(x)$.

Proof. To say that $\left.\mathscr{E}\right|_{X_{v}}$ has depth equal to $t$ at $x$ is equivalent to asserting that $\operatorname{depth}_{x} \mathscr{E}=$ $\left(\operatorname{depth}_{\mathscr{O}_{V, v}} \mathscr{O}_{V, v}\right)+t$, by Proposition A.3. The result follows.

The following vanishing allows us to extend sections over sets of relative codimension 2 .

Proposition A.6 ([HK04, Proposition 3.3]). Let $f: X \rightarrow V$ be a morphism of Noetherian schemes and $\mathscr{E}$ a coherent sheaf on $X$, flat and $S_{r}$ over $V$. Let $Z \subseteq X$ be a closed subscheme of $X$ such that we have codim $\operatorname{Supp}_{\mathscr{E}}\left(\operatorname{Supp} \mathscr{E}_{v} \cap Z_{v}\right) \geqslant r$ for every $v \in \bar{V}$. Then we have $\mathscr{H}_{Z}^{i}(\mathscr{E})=0$ for each $0 \leqslant i<r$.

Proof. We follow the proof in [HK04, Proposition 3.3]. First, we certainly have a spectral sequence $E_{2}^{p, q}:=H_{x}^{p} \circ \mathscr{H}_{Z}^{q} \Rightarrow H_{x}^{p+q}$. We then induct on $r$, the base case of $r=0$ being trivial. Now, assume $\mathscr{H}_{Z}^{0}(\mathscr{E})=\ldots=\mathscr{H}_{Z}^{r-2}(\mathscr{E})=0$ but $\mathscr{H}_{Z}^{r-1}(\mathscr{E}) \neq 0$. Then, for any $x \in Z$ which is a generic point ${ }^{10}$ of the support of $\mathscr{H}_{Z}^{r-1}(\mathscr{E})$, we have $H_{x}^{0}\left(\mathscr{H}_{Z}^{r-1}(\mathscr{E})\right) \neq 0$ (note here that $x$ is probably not a closed point). Additionally, since $x$ is a point of $Z \cap \operatorname{Supp}(\mathscr{E})$, we have $\operatorname{dim}\left(\left.\mathscr{E}\right|_{X_{v}}\right)_{x} \geqslant r$.

On the other hand, for all $i>0$, we have $H_{x}^{i}\left(\mathscr{H}_{Z}^{r-1-i}(\mathscr{E})\right)=0$, and so by the spectral sequence, $H_{x}^{r-1}(\mathscr{E}) \cong H_{x}^{0}\left(\mathscr{H}_{Z}^{r-1}(\mathscr{E})\right) \neq 0$. This contradicts Corollary A.5.

We now obtain a relative version of Hartog's phenomena, just as in [HK04].

Proposition A.7 ([HK04, Proposition 3.5, 3.6.1]). Let $f: X \rightarrow V$ be a morphism of Noetherian schemes and $\mathscr{E}$ a coherent sheaf on $X$ which satisfies one of the following two conditions:

(a) either $\mathscr{E}$ is reflexive and $f$ is flat and relatively $S_{2}$,

(b) or $\mathscr{E}$ is of full support, flat and $S_{2}$ over $V$.

Let $j: U \hookrightarrow X$ be an open set such that for $Z:=X \backslash U$, we have $\operatorname{codim}_{X_{v}} Z_{v} \geqslant 2$ for every $v \in V$. Then the following natural map is an isomorphism:

$$
\left.\mathscr{E} \rightarrow j_{*} \mathscr{E}\right|_{U}
$$

Proof. First, assume that $\mathscr{E}$ is reflexive. Consider a presentation of $\mathscr{E}^{*}$ by locally free sheaves

$$
\mathscr{E}_{2} \longrightarrow \mathscr{E}_{1} \longrightarrow \mathscr{E}^{*} \longrightarrow 0 \text { and the dual } 0 \longrightarrow \mathscr{E}^{\longrightarrow} \mathscr{E}_{1}^{*} \longrightarrow \mathscr{E}_{2}^{*}
$$

\footnotetext{
${ }^{10} \mathrm{~A}$ generic point is a minimal prime in the language of commutative algebra.
} 


\section{$F$-SINGULARITIES IN FAMILIES}

Then, applying Proposition A.6 to $\mathscr{O}_{X}$ and then applying local cohomology to the above exact sequence yields

$$
\mathscr{H}_{Z}^{1}(\mathscr{E})=\mathscr{H}_{Z}^{0}(\mathscr{E})=0 .
$$

If, instead, $\mathscr{E}$ is flat and S2 and has full support, then we still have the vanishing (A.1) by Proposition A.6. Consider then the exact sequence

$$
0 \longrightarrow \mathscr{H}_{Z}^{0}(\mathscr{E}) \longrightarrow \mathscr{E} \longrightarrow j_{*} \mathscr{E} \longrightarrow \mathscr{H}_{Z}^{1}(\mathscr{E})
$$

Applying (A.1) concludes our proof.

Corollary A.8 ([HK04, Proposition 3.6.2]). Let $f: X \rightarrow V$ be a flat, $S_{2}$ morphism of Noetherian schemes, $\mathscr{E}$ a coherent sheaf on $X$ satisfying either part (a) or part (b) of Proposition A.7. Let $\mathscr{F}$ be another coherent sheaf satisfying part (a) or (b) of that proposition. Let $j: U \hookrightarrow X$ be an open set such that for $Z:=X \backslash U$, we have $\operatorname{codim}_{X_{v}} Z_{v} \geqslant 2$ for every $v \in V$. Also assume that $\left.\left.\mathscr{E}\right|_{U} \cong \mathscr{F}\right|_{U}$. Then $\mathscr{E} \cong \mathscr{F}$.

Proof. In any case, $\left.j_{*} \mathscr{E}\right|_{U}=\mathscr{E}$ and $\left.j_{*} \mathscr{F}\right|_{U}=\mathscr{F}$, and so the result follows.

Proposition A.9 ([HK04, Corollary 3.7]). Let $f: X \rightarrow V$ be a flat, $S_{2}$ morphism of Noetherian schemes and $j: U \hookrightarrow X$ an open set such that for $Z:=X \backslash U$, we have $\operatorname{codim}_{X_{v}} Z_{v} \geqslant 2$ for every $v \in V$. Also assume that $\mathscr{E}$ is a reflexive, coherent sheaf on $U$. Then $j_{*} \mathscr{E}$ is reflexive.

Proof. Choose a coherent subsheaf $\mathscr{F} \subseteq j_{*} \mathscr{E}$ such that $\left.\mathscr{F}\right|_{U}=\mathscr{E}$ [Har77, Exercise II.5.15]. Then $j_{*} \mathscr{E}$ is reflexive by the following computation:

$$
\mathscr{F}^{* *} \cong \underbrace{j_{*}\left(\left.\mathscr{F}^{* *}\right|_{U}\right)}_{\text {by Proposition A.7 }} \cong \underbrace{j_{*} \mathscr{E}^{* *}}_{\left.\mathscr{E} \cong \mathscr{F}\right|_{U}} \cong \underbrace{j_{*} \mathscr{E}}_{\mathscr{E} \text { is reflexive }}
$$

This completes the proof.

Proposition A.10. If $f: X \rightarrow Y$ is a projective, flat, relatively $S 2$ and G1, equidimensional morphism, then $\omega_{X / Y}$ is reflexive.

Proof. The proof given in [PS14, Lemma 4.8] works.

\section{ACKNOWLEDGEMENTS}

The authors began working on this project while attending a workshop at the American Institute of Mathematics (AIM) titled "ACC for minimal log discrepancies and termination of flips," May 14th - 18th 2012, organized by Tommaso de Fernex and Christopher Hacon. We would like to thank the organizers and the American Institute of Mathematics. Part of the work was done when the third author visited the Department of Mathematics at Pennsylvania State University; he would like to thank them for their hospitality. Additionally, the authors would like to thank Lawrence Ein, Christopher Hacon, János Kollár, Joseph Lipman, Mircea Mustață, and Kevin Tucker for useful discussions. Especially, we would like to thank Brian Conrad for pointing out the compatibility of the trace map with base change. We would also like to thank Hiromu Tanaka for comments on a previous draft. Additionally, the authors of the paper would like to thank Leo Alonso, Damian Rössler, Graham Leuschke, and Blup for providing answers and commentary to the following questions: http://mathoverflow.net/questions/120982/ and http://mathoverflow. net/questions/120625/. 


\section{Z. Patakfalvi, K. Schwede And W. Zhang}

\section{REFERENCES}

And93 M. André, Homomorphismes réguliers en caractt́ouristic p, C. R. Acad. Sci. Paris Sér. I Math. 316 (1993), no. 7, 643-646.

BB11 M. Blickle and G. Böckle, Cartier modules: Finiteness results, J. reine angew. Math. 661 (2011), 85-123; https://doi.org/10.1515/CRELLE.2011.087.

BK05 M. Brion and S. Kumar, Frobenius splitting methods in geometry and representation theory, Progr. Math., vol. 231 (Birkhäuser Boston, Inc., Boston, MA, 2005); https://doi.org/10. $1007 / \mathrm{b} 137486$.

Bli13 M. Blickle, Test ideals via algebras of $p^{-e}$-linear maps, J. Algebraic Geom. 22 (2013), no. 1, 49-83; https://doi.org/10.1090/S1056-3911-2012-00576-1.

BMS08 M. Blickle, M. Mustaţă, and K. E. Smith, Discreteness and rationality of F-thresholds, Michigan Math. J. 57 (2008), 43-61; https://doi.org/10.1307/mmj/1220879396.

BS13 M. Blickle and K. Schwede, $p^{-1}$-linear maps in algebra and geometry, Commutative Algebra: Expository Papers Dedicated to David Eisenbud on the Occasion of his 65th Birthday, ed. I. Peeva (Springer, New York, 2013), 123-205; https://doi.org/10.1007/ 978-1-4614-5292-8_5.

BST15 M. Blickle, K. Schwede, and K. Tucker, F-singularities via alterations, Amer. J. Math. 137 (2015), no. 1, 61-109; https://doi.org/10.1353/ajm.2015.0000.

BSTZ10 M. Blickle, K. Schwede, S. Takagi, and W. Zhang, Discreteness and rationality of F-jumping numbers on singular varieties, Math. Ann. 347 (2010), no. 4, 917-949; https://doi.org/10. 1007/s00208-009-0461-2.

CHMS14 P. Cascini, C. Hacon, M. Mustaţă, and K. Schwede, On the numerical dimension of pseudoeffective divisors in positive characteristic, Amer. J. Math. 136 (2014), no. 6, 1609-1628; https: //doi.org/10.1353/ajm.2014.0047.

Con00 B. Conrad, Grothendieck duality and base change, Lecture Notes in Math., vol. 1750 (SpringerVerlag, Berlin, 2000); https://doi.org/10.1007/b75857.

Eis95 D. Eisenbud, Commutative algebra. with a view toward algebraic geometry, Grad. Texts in Math., vol. 150 (Springer-Verlag, New York, 1995); https://doi.org/10.1007/ 978-1-4612-5350-1.

Fed83 R. Fedder, F-purity and rational singularity, Trans. Amer. Math. Soc. 278 (1983), no. 2, 461480; https://doi.org/10.2307/1999165.

FST11 O. Fujino, K. Schwede, and S. Takagi, Supplements to non-lc ideal sheaves, Higher Dimensional Algebraic Geometry, RIMS Kôkyûroku Bessatsu, vol. B24 (Res. Inst. Math. Sci., Kyoto, 2011), $1-46$.

Gab04 O. Gabber, Notes on some t-structures, Geometric Aspects of Dwork Theory, Vol. I, II, (Walter de Gruyter, Berlin, 2004), 711-734.

Gro67 A. Grothendieck, Éléments de géométrie algébrique. IV. Étude locale des schémas et des morphismes de schémas, Quatrième partie, Publ. Math. Inst. Hautes Études Sci. (1967), no. 32, 5-361; http://www. numdam.org/item?id=PMIHES_1967_-_32__5_0.

GW77 S. Goto and K. Watanabe, The structure of one-dimensional F-pure rings, J. Algebra 49 (1977), no. 2, 415-421; https://doi.org/10.1016/0021-8693(77)90250-2.

Hac15 C. D. Hacon, Singularities of pluri-theta divisors in Char $p>0$, Algebraic Geometry in East Asia - Taipei 2011, Adv. Stud. Pure Math., vol. 65 (Math. Soc. Japan, Tokyo, 2015), 117-122.

Har98 N. Hara, A characterization of rational singularities in terms of injectivity of Frobenius maps, Amer. J. Math. 120 (1998), no. 5, 981-996; https://doi.org/10.1353/ajm.1998.0037.

Har77 R. Hartshorne, Algebraic geometry, Grad. Texts in Math., vol. 52 (Springer-Verlag, New YorkHeidelberg, 1977); https://doi.org/10.1007/978-1-4757-3849-0.

Har94_, Generalized divisors on Gorenstein schemes, K-Theory 8 (1994), no. 3, 287-339; https://doi.org/10.1007/BF00960866. 


\section{$F$-SINGULARITIES IN FAMILIES}

Has01 M. Hashimoto, Cohen-Macaulay F-injective homomorphisms, Geometric and Combinatorial Aspects of Commutative Algebra (Messina, 1999), Lect. Notes Pure Appl. Math., vol. 217 (Dekker, New York, 2001), 231-244.

Has10 _ F-pure homomorphisms, strong F-regularity, and F-injectivity, Comm. Algebra 38 (2010), no. 12, 4569-4596; https://doi.org/10.1080/00927870903431241.

HH94 M. Hochster and C. Huneke, F-regularity, test elements, and smooth base change, Trans. Amer. Math. Soc. 346 (1994), no. 1, 1-62; https://doi.org/10.2307/2154942.

HK04 B. Hassett and S. J. Kovács, Reflexive pull-backs and base extension, J. Algebraic Geom. 13 (2004), no. 2, 233-247; https://doi.org/10.1090/S1056-3911-03-00331-X.

Hoc07 M. Hochster, Foundations of tight closure theory, 2007, Lecture notes from a course taught on the University of Michigan Fall 2007, available at http://www.math.1sa.umich.edu/ hochster/711F07/fndtc.pdf.

HR76 M. Hochster and J. L. Roberts, The purity of the Frobenius and local cohomology, Adv. Math. 21 (1976), no. 2, 117-172; https://doi.org/10.1016/0001-8708(76)90073-6.

HS77 R. Hartshorne and R. Speiser, Local cohomological dimension in characteristic p, Ann. of Math. 105 (1977), no. 1, 45-79; https ://doi.org/10.2307/1971025.

HS06 C. Huneke and I. Swanson, Integral closure of ideals, rings, and modules, London Math. Soc. Lect. Note Ser., vol. 336 (Cambridge Univ. Press, Cambridge, 2006).

HX15 C.D. Hacon and C. Xu, On the three dimensional minimal model program in positive characteristic, J. Amer. Math. Soc. 28 (2015), no. 3, 711-744; https://doi.org/10.1090/ S0894-0347-2014-00809-2.

HY03 N. Hara and K.-I. Yoshida, A generalization of tight closure and multiplier ideals, Trans. Amer. Math. Soc. 355 (2003), no. 8, 3143-3174; https://doi.org/10.1090/ S0002-9947-03-03285-9.

KAAC92 J. Kollár, D. Abramovich, V. Alexeev, and A. Corti (eds), Flips and abundance for algebraic threefolds, Papers from the Second Summer Seminar on Algebraic Geometry (University of Utah, August 1991), Astérisque, no. 211 (Soc. Math. France, Paris, 1992).

Kat08 M. Katzman, Parameter-test-ideals of Cohen-Macaulay rings, Compos. Math. 144 (2008), no. 4, 933-948; https://doi.org/10.1112/S0010437X07003417.

Kee03 D. S. Keeler, Ample filters of invertible sheaves, J. Algebra 259 (2003), no. 1, 243-283; https: //doi.org/10.1016/S0021-8693(02)00557-4.

Kob75 N. Koblitz, p-adic variation of the zeta-function over families of varieties defined over finite fields, Compos. Math. 31 (1975), no. 2, 119-218; http://www . numdam.org/item?id=CM_1975_ _31_2_119_0.

Kol90 J. Kollár, Projectivity of complete moduli, J. Differential Geom. 32 (1990), no. 1, 235-268; http://dx.doi.org/10.4310/jdg/1214445046.

Kun69 E. Kunz, Characterizations of regular local rings for characteristic p, Amer. J. Math. 91 (1969), 772-784; https://doi.org/10.2307/2373351.

Kun86_ Kähler differentials, Adv. Lect. Math. (Friedr. Vieweg \& Sohn, Braunschweig, 1986); https ://doi.org/10.1007/978-3-663-14074-0.

Laz04a R. Lazarsfeld, Positivity in algebraic geometry. I. Classical setting: Line bundles and linear series, Ergeb. Math. Grenzgeb. (3), vol. 48 (Springer-Verlag, Berlin, 2004); https : //doi .org/ 10.1007/978-3-642-18808-4.

Laz04b _ Positivity in algebraic geometry. II. Positivity for vector bundles, and multiplier ideals, Ergeb. Math. Grenzgeb. (3), vol. 49 (Springer-Verlag, Berlin, 2004); https://doi.org/10. 1007/978-3-642-18808-4.

Lyu97 G. Lyubeznik, F-modules: Applications to local cohomology and D-modules in characteristic $p>0$, J. reine angew. Math. 491 (1997), 65-130; https://doi.org/10.1515/crl1.1997. 491.65 . 


\section{Z. Patakfalvi, K. Schwede And W. Zhang}

Mil72 L. Miller, Curves with invertible Hasse-Witt-matrix, Math. Ann. 197 (1972), 123-127; https: //doi.org/10.1007/BF01419588.

MR85 V.B. Mehta and A. Ramanathan, Frobenius splitting and cohomology vanishing for Schubert varieties, Ann. of Math. 122 (1985), no. 1, 27-40; https://doi.org/10.2307/1971368.

MS97 V. B. Mehta and V. Srinivas, A characterization of rational singularities, Asian J. Math. 1 (1997), no. 2, 249-271; https://doi.org/10.4310/AJM.1997.v1.n2.a4.

MS12 L. E. Miller and K. Schwede, Semi-log canonical vs F-pure singularities, J. Algebra 349 (2012), 150-164; https://doi.org/10.1016/j.jalgebra.2011.08.035.

MS14 M. Mustaţă and K. Schwede, A Frobenius variant of Seshadri constants, Math. Ann. 358 (2014), no. 3-4, 861-878; https://doi.org/10.1007/s00208-013-0976-4.

Mus13 M. Mustaţă, The non-nef locus in positive characteristic, A Celebration of Algebraic Geometry, Clay Math. Proc., vol. 18 (Amer. Math. Soc., Providence, RI, 2013), 535-551.

MY09 M. Mustaţă and K.-I. Yoshida, Test ideals vs. multiplier ideals, Nagoya Math. J. 193 (2009), 111-128; http://projecteuclid.org/euclid.nmj/1236089983.

Pat14 Z. Patakfalvi, Semi-positivity in positive characteristics, Ann. Sci. Éc. Norm. Supér. (4) 47 (2014), no. 5, 991-1025; https://doi.org/10.24033/asens.2232.

PS14 Z. Patakfalvi and K. Schwede, Depth of F-singularities and base change of relative canonical sheaves, J. Inst. Math. Jussieu 13 (2014), no. 1, 43-63; https://doi.org/10.1017/ S1474748013000066.

Rad92 N. Radu, Une classe d'anneaux noethériens, Rev. Roumaine Math. Pures Appl. 37 (1992), no. $1,79-82$.

RR85 S. Ramanan and A. Ramanathan, Projective normality of flag varieties and Schubert varieties, Invent. Math. 79 (1985), no. 2, 217-224; https://doi.org/10.1007/BF01388970.

Sch09 K. Schwede, F-adjunction, Algebra Number Theory 3 (2009), no. 8, 907-950; https://doi. org/10.2140/ant.2009.3.907.

Sch11 , Test ideals in non- $\mathbb{Q}$-Gorenstein rings, Trans. Amer. Math. Soc. 363 (2011), no. 11, 5925-5941; https://doi.org/10.1090/S0002-9947-2011-05297-9.

Sch14 A A canonical linear system associated to adjoint divisors in characteristic $p>0$, J. reine angew. Math. 696 (2014), 69-87; https://doi.org/10.1515/crelle-2012-0087.

Sha07 R.Y. Sharp, On the Hartshorne-Speiser-Lyubeznik theorem about Artinian modules with a Frobenius action, Proc. Amer. Math. Soc. 135 (2007), no. 3, 665-670; https://doi.org/ 10.1090/S0002-9939-06-08606-0.

Smi97 K.E. Smith, F-rational rings have rational singularities, Amer. J. Math. 119 (1997), no. 1, 159-180; https://doi.org/10.1353/ajm.1997.0007.

Sri91 V. Srinivas, Normal surface singularities of F-pure type, J. Algebra 142 (1991), no. 2, 348-359; https://doi.org/10.1016/0021-8693(91)90311-U.

ST12 K. Schwede and K. Tucker, A survey of test ideals, Progress in Commutative Algebra 2, Closures, Finiteness and Factorization (C. Francisco, L.C. Klinger, S.M. Sather-Wagstaff, and J.C. Vassilev, eds) (Walter de Gruyter, Berlin, 2012), 39-99.

Sta17 The Stacks Project, 2017; http://stacks.math.columbia.edu.

SZ09 K. Shimomoto and W. Zhang, On the localization theorem for F-pure rings, J. Pure Appl. Algebra 213 (2009), no. 6, 1133-1139; https://doi.org/10.1016/j.jpaa.2008.11.047.

Tak04 S. Takagi, An interpretation of multiplier ideals via tight closure, J. Algebraic Geom. 13 (2004), no. 2, 393-415, https://doi.org/10.1090/S1056-3911-03-00366-7.

Tan15 H. Tanaka, The trace map of Frobenius and extending sections for threefolds, Michigan Math. J. 64 (2015), no. 2, 227-261; https://doi.org/10.1307/mmj/1434731922.

Tan72 H. Tango, On the behavior of extensions of vector bundles under the Frobenius map, Nagoya Math. J. 48 (1972), 73-89; https://doi.org/10.1017/S0027763000015099. 


\section{$F$-SINGULARITIES IN FAMILIES}

Vie95 E. Viehweg, Quasi-projective moduli for polarized manifolds, Ergeb. Math. Grenzgeb. (3), vol. 30 (Springer-Verlag, Berlin, 1995); https://doi.org/10.1007/978-3-642-79745-3.

Zha14 Y. Zhang, Pluri-canonical maps of varieties of maximal Albanese dimension in positive characteristic, J. Algebra 409 (2014), 11-25; https://doi.org/10.1016/j.jalgebra.2014.03.019.

Zsolt Patakfalvi zsolt.patakfalvi@epfl.ch

EPFL, SB MATHGEOM CAG, MA B3 635 (Bâtiment MA), Station 8, CH-1015 Lausanne, Switzerland

Karl Schwede schwede@math.utah.edu

Department of Mathematics, University of Utah, Salt Lake City, UT 84112, USA

Wenliang Zhang wlzhang@uic.edu

Department of Mathematics, University of Illinois at Chicago, Chicago, IL 60607, USA 